Outbreak Investigation Report

A community-based gastroenteritis outbreak after Typhoon Haiyan, Leyte, Philippines 1

Ventura RJ, Muhi E, de los Reyes VC, Sucaldito MN, Tayag E

Surveillance Reports

Tuberculosis case notification data in Viet Nam, 2007 to 2013

Nguyen NV, Nguyen HB, Pham KH, Hennig C

Tuberculosis case finding in Cambodia: analyis of case notification data, 2000 to 2013

Morishita F, Furphy VB, Kobayashi M, Nishikiori N, Eang MT, Yadav RP

Original Researches

Mass poisoning after consumption of a hawksbill turtle, Federated States of Micronesia, 2010

Pavlin BI, Musto J, Pretrick M, Sarofalpiy J, Sappa P,

Shapucy S, Kool JL

Hospital preparedness for Ebola virus disease: a training course in the Philippines

Carlos C, Capistrano R, Borja-Tobora CF, delos Reyes MR, Lupisan S, Corpuz A, Aumentado C, Lee Suy L, Hall J, Donald J, Counahan M, Curless MS, Rhymer W, Gavin M, Lynch C, Black MA, Anduyon AD, Buttner $P$, Speare $R$

Intestinal parasites of children and adults in a remote Aboriginal community of the Northern Territory, Australia, 1994-1996 Shield J, Aland K, Kearns T, Gongdjalk G, Holt D, Currie B, Provic $P$

\section{Brief Reports}

Brief behavioural surveys in routine HIV sentinel surveillance: a new tool for monitoring the HIV epidemic in Viet Nam

Duong CT, Nguyen TH, Nguyen AT, Hoang THH, Pham HT, Nguyen TTH, Le AT, Tran DQ, Tran HT, Nguyen LH, Phan TTH, Vo HS, Bui HD, Nguyen TN, Jacka D, Sabin $K$

\section{Sexually transmitted infections among} transgender people and men who have sex with men

Veronese V, van Gemert C, Bulu S, Kwarteng T, Bergeri I,
Case Reports

Identification of Enterovirus C105 for the first time in New Zealand

Todd A, Taylor S, Huang QS

International Health Regulations (2005) facilitate communication for in-flight contacts of a Middle East respiratory syndrome case, Hong Kong Special Administrative Region, 2014

Poon KM, Wong ML, Leung YH, Sin KW, To MKL, Chuang SK

Regional Analysis

Ebola preparedness in the Western Pacific Region, 2014

$X u Z$, Pavlin Bl, Squires RC, Chinnayah T, Konings $F$,

\title{
Western Pacific Surveillance and Response
}

Open access journal with continuous publication

Western Pacific Surveillance and Response (WPSAR) is an open access journal dedicated to the surveillance of and response to public health events. The goal of the journal is to create a platform for timely information sharing both within our region and globally to enhance surveillance and response activities. WPSAR is a continuous publication which means articles will be published online as soon as they have completed the review and editing process. Every three months articles will be batched for a print issue. It is a publication managed by the World Health Organization Regional Office for the Western Pacific.

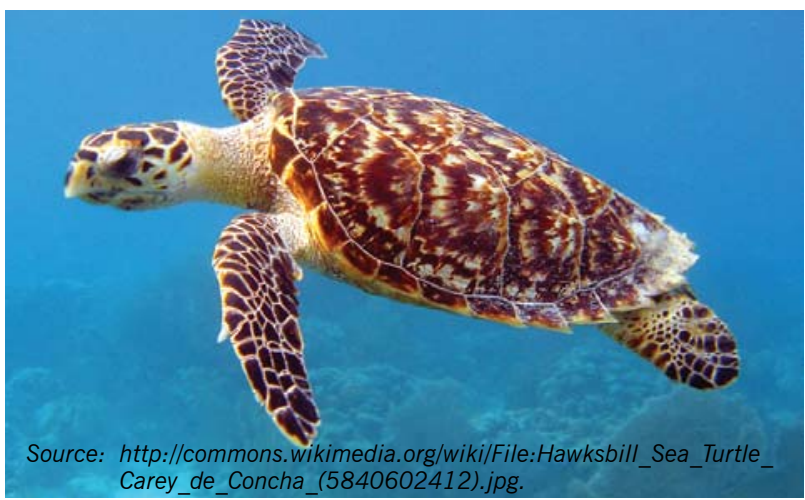




\title{
EDITORIAL TEAM
}

\section{Copyright notice}

(c) World Health Organization 2010

p-ISSN: 2094-7321

e-ISSN: 2094-7313

Ailan Li

Executive Editor

Joy Gregory

Michelle McPherson

Coordinating Editors

Elizabeth Mangali

Assistant Editor

Associate Editors

Jorge Mendoza Aldana

Frank Konings

Nobuyuki Nishikiori

Manju Rani

Boris Pavlin

Dongbao Yu

\section{To contact us:}

\author{
Western Pacific Surveillance and Response \\ World Health Organization \\ Office for the Western Pacific Region \\ United Nations Avenue \\ 1000 Manila, Philippines \\ wpsar@wpro.who.int \\ www.wpro.who.int/wpsar
}

All rights reserved. The information presented in the various pages of this journal is issued by the World Health Organization for general distribution, and is protected under the Berne Convention for the Protection of Literature and Artistic Works, under national laws on copyright and neighbouring rights.

The World Health Organization does not warrant that the information contained in this publication is complete and correct and shall not be liable for any damages incurred as a result of its use.

Publications of the World Health Organization can be obtained from Marketing and Dissemination, World Health Organization, 20 Avenue Appia, 1211 Geneva 27, Switzerland (tel.: +41 22791 3264; fax: +41 22791 4857; email: bookorders@who.int). Requests for permission to reproduce WHO publications, in part or in whole, or to translate them - whether for sale or for non-commercial distribution should be addressed to Publications, at the above address (+41 22791 4806; e-mail: permissions@who.int). For WHO Western Pacific Regional Publications, request for permission to reproduce should be addressed to Publications Office, World Health Organization, Regional Office for the Western Pacific, P.O. Box 2932, 1000, Manila, Philippines, fax: +632 521 1036, e-mail: publications@wpro.who.int.

\section{Disclaimer}

The designations employed and the presentation of the information in this publication do not imply the expression of any opinion whatsoever on the part of the World Health Organization concerning the legal status of any country, territory, city or area or of its authorities, or concerning the delimitation of its frontiers or boundaries.

The mention of specific companies or of certain manufacturers' products does not imply that they are endorsed or recommended by the World Health Organization in preference to others of a similar nature that are not mentioned. Errors and omissions excepted, the names of proprietary products are distinguished by initial capital letters. 


\title{
A community-based gastroenteritis outbreak after Typhoon Haiyan, Leyte, Philippines, 2013
}

\author{
Ray Justin Ventura, ${ }^{a}$ Edzel Muhi, ${ }^{b}$ Vikki Carr de los Reyes, ${ }^{a}$ Ma Nemia Sucaldito and Enrique Tayag ${ }^{a}$ \\ Correspondence to Ray Justin Ventura (e-mail: rayjustinventura@gmail.com).
}

Background: Three weeks after Typhoon Haiyan, an increasing number of acute gastroenteritis cases were reported in Kananga, Leyte, an area where evacuated residents had returned home two days after the disaster. An outbreak investigation was conducted to identify the source and risk factors associated with the increase of gastroenteritis.

Methods: A case was defined as any person in Kananga who developed acute diarrhoea ( $\geq 3$ times/24 hours) and any of the following symptoms: fever, nausea, vomiting or abdominal pain from 11 November 2013 to 10 December 2013. Active case finding was conducted by reviewing medical records, and a case-control study was conducted. Rectal swabs and water samples were tested for bacteriological examination.

Results: One hundred and five cases were identified. Multivariate analysis revealed that consumption of untreated drinkingwater was associated with illness (adjusted odds ratio: 18.2). Both rectal swabs and municipal water samples tested positive for Aeromonas hydrophila. On inspection of the municipal water system, breaks in the distribution pipes were found with some submerged in river water.

Conclusion: This acute gastroenteritis outbreak was most likely caused by Aeromonas hydrophila and transmitted through a contaminated water source. This study highlights that areas less damaged by a disaster that do not require ongoing evacuation centres can still have acute gastroenteritis outbreaks. All affected areas should be monitored during a disaster response, not just those with evacuation centres. Boiling or chlorinating of water should also be recommended for all areas affected by disaster.

T yphoon Haiyan swept through the central Philippines on 8 November 2013, killing over 6000 and displacing 4 million people; it damaged schools, health centres, other infrastructure and 1.1 million houses. ${ }^{1}$

In western Leyte, the typhoon affected 18 municipalities with a total of 884546 people affected. Unlike other areas affected by the typhoon, evacuation centres in Kananga, Leyte, decamped two days after the typhoon with all evacuees returning to their own houses. Although western Leyte appeared to have suffered less than eastern Leyte and Samar, ${ }^{1}$ a lack of disease surveillance reports from this area was a concern. Therefore, active surveillance was established at the Kananga Municipal Hospital and Kananga Rural Health Unit, which reported on vector-borne diseases, tetanus and diarrhoea.

Three weeks after Typhoon Haiyan, an increasing number of acute gastroenteritis cases were reported in Kananga, Leyte, through this active surveillance system. The 60 cases reported in November 2013 were $757 \%$ higher than the same month the previous year. ${ }^{2}$ Therefore, a team from the Department of Health was sent to conduct an outbreak investigation to identify the source and risk factors associated with the increase of gastroenteritis.

\section{METHODS}

\section{Epidemiological investigation}

A case was defined as any person in Kananga, Leyte with acute diarrhoea ( $\geq 3$ times/24 hours) and any of the following signs and symptoms: fever, nausea, vomiting and abdominal pain from 11 November to 10 December 2013. Active case finding was conducted by reviewing medical records from Kananga Rural Health Unit and Kananga Municipal Hospital. The initial 10 cases were interviewed using a structured questionnaire which included questions on food and

\footnotetext{
Department of Health, Sta Cruz, Manila, Philippines.

Mogpog Municipal Health Office, Marinduque, Philippines. Submitted: 13 February 2014; Published: 10 January 2015 doi: 10.5365/wpsar.2014.5.1.010
} 
Figure 1. Acute gastroentiritis cases by date of onset of illness, Kananga, Leyte, 11 November-10 December 2013 $(n=105)$

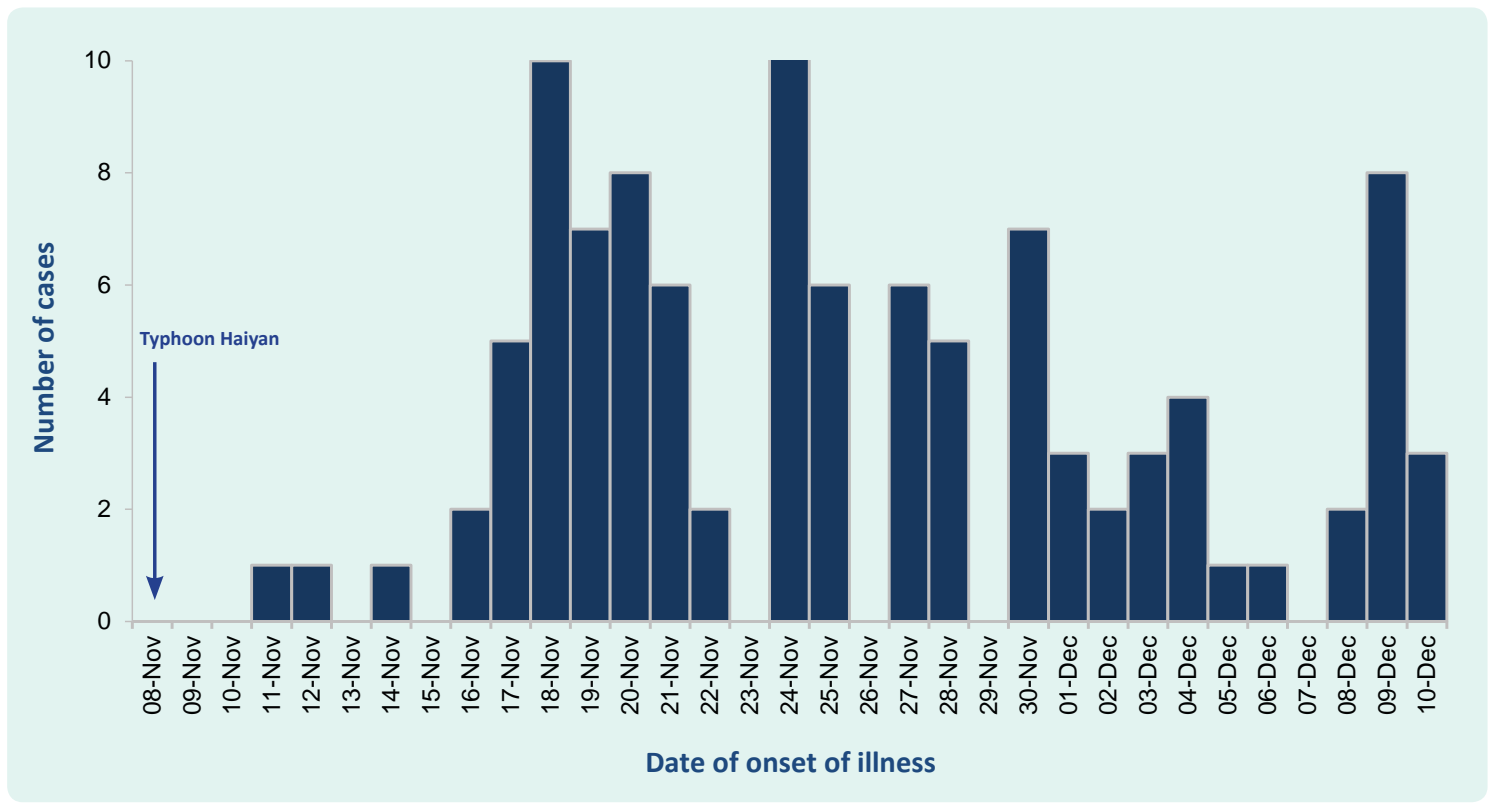

water exposures and other environmental risk factors. A map showing attack rates and water supply by village was constructed using ArcGIS (Redlands, CA, USA).

An unmatched case-control study with a planned 1:2 ratio of cases to controls was conducted to test the hypothesis. Not all cases were included due to logistical barriers with purposive selection of cases. Controls were individuals who resided in the same or nearby households to the case; they were excluded if they reported any gastrointestinal symptoms or if they tested positive from bacterial examination. A more specific questionnaire was used for the case-control study that focused on drinkingwater, hygiene practices and other environmental factors. These exposures were then compared, and analysis was conducted using EPI info version 3.5.4. We calculated odds ratios (OR) and confidence intervals. A forward stepwise procedure was used in developing a model for the multivariate analysis which included variables that were significant in the bivariate analysis.

\section{Laboratory investigation}

Rapid diagnostic test was conducted using Denka (Japan) for rotavirus and CTK (San Diego, CA, USA) for norovirus. Rectal swabs were collected for both cases and controls, as well as for a family that was living near the water supply, using Cary-Blair media for standard bacterial culture testing.
A single water sample $(500 \mathrm{ml})$ was collected from each of the three intake tanks and two reservoirs of the municipal water system. Six water sources connected to the municipal water system were chosen purposively for collection of a single water sample $(500 \mathrm{ml})$ (Village Poblacion, Village Natubgan and Village Lonoy). The specimens were sent to Research Institute for Tropical Medicine, Mutinlupa City, the Philipines for bacterial analysis. The Swiss humanitarian aid team, which was conducting relief efforts after Typhoon Haiyan, also tested three water sources connected to the municipal water system for residual chlorine (Reservoire, Village Natubgan and Village Poblacion)

\section{Environmental investigation}

Environmental investigation was conducted in the three villages with the highest number of cases that were using the municipal water system.

\section{RESULTS}

\section{Cases}

A total of 105 cases were identified. The first onset date was 11 November 2013 with a peak from 17 to 20 November (Figure 1). The majority (101 or $96 \%)$ reported watery diarrhoea with four cases (4\%) reporting bloody diarrhoea. Other signs and symptoms 
Figure 2. Attack rate and water supply by village, Kananga, Leyte, 11 November-10 December 2013 $(n=105)$

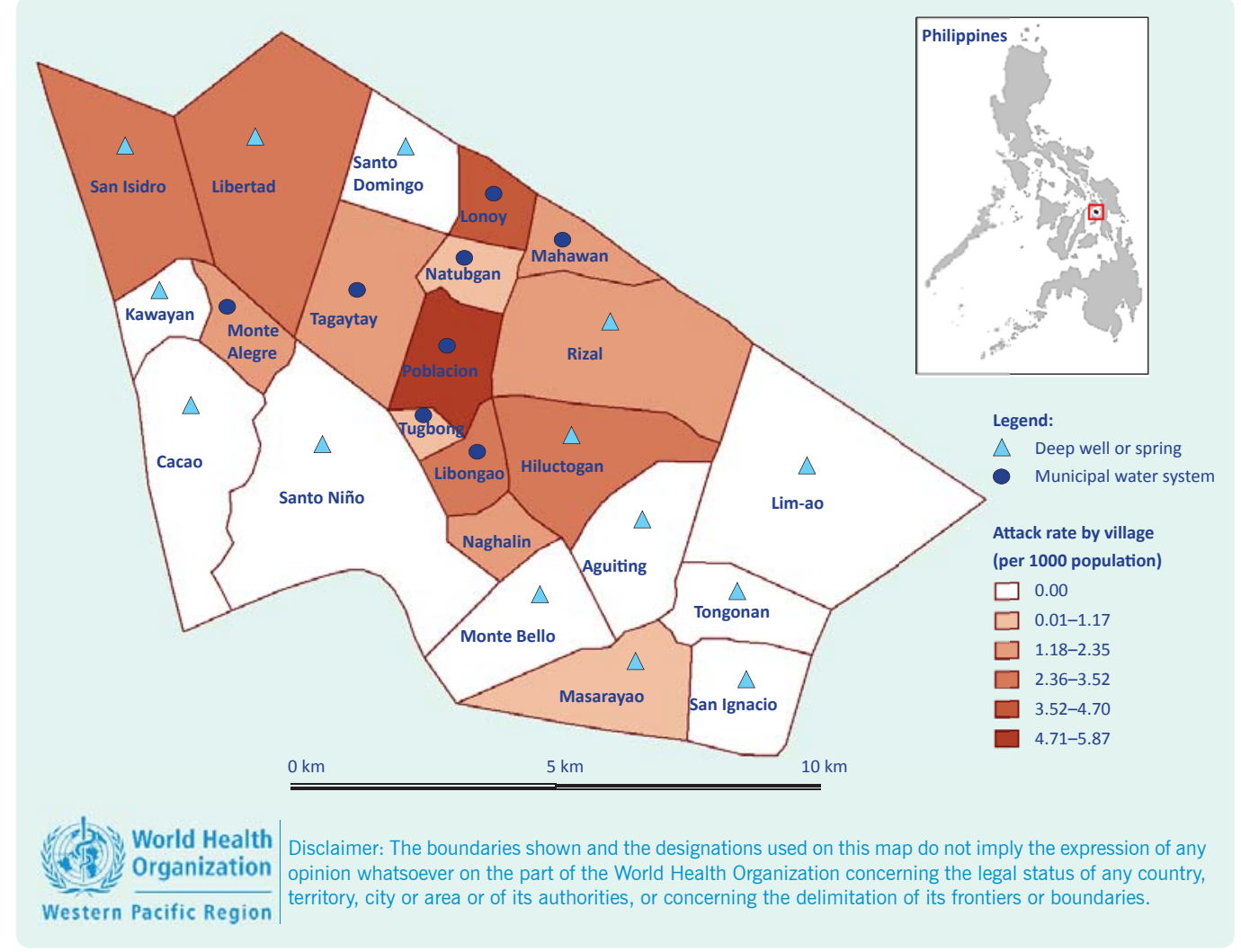

included vomiting $(51 \%)$, fever $(47 \%)$ and abdominal pain (32\%). Forty-eight cases (46\%) were hospitalized and no deaths were reported. Nineteen of the cases reported antibiotic use before laboratory testing.

The age of cases ranged from 4 months to 61 years (median $=2$ years) with $51 \%$ male. The most affected age group was 1 to 5 years (49\%). Poblacion village had the highest attack rate (AR) ( $A R=6 / 1000$ population) followed by Barangay Lonoy $(A R=4.46 / 1000$ population), both of which were supplied water through the municipal water system (Figure 2). Twenty-five out of 39 cases interviewed used the municipal water system; $72 \%$ were not disinfecting the water.

\section{Case-control study}

The case-control study comprised 39 cases and 61 controls. None of the 61 selected controls tested positive on bacterial examination. Bivariate analysis revealed three significant risk factors: drinking untreated water (not chlorinated or boiled, mostly municipal water), no access to toilet facilities and using deep wells or springs as a drinking-water source. After multivariate analysis, only drinking untreated water remained associated with being a case and the adjusted OR of 18.17 was similar to the crude OR of 22.15 (Table 1 ).

\section{Laboratory investigation}

The four specimens tested for rotavirus and norovirus were negative. Of the 39 rectal swab specimens collected for cases, 11 (28\%) were positive for the following bacterial pathogens: Aeromonas hydrophila (four or 10\%), Campylobacter jejuni (three or 8\%), Campylobacter coli (three or $8 \%$ ) and Shigella sonnei (one or 3\%). All 61 controls selected had negative rectal swabs for bacterial culture. All four cases that tested positive for Aeromonas hydrophila had the municipal water system as their source of drinking-water.

A total of 11 water samples were collected and three $(27 \%)$ were positive for Aeromonas hydrophila (two intake tanks of the municipal water system and a household in Village Natubgan). No residual chlorine was detected in the three water sources tested. 


\begin{tabular}{|c|c|c|c|c|c|c|}
\hline \multirow{2}{*}{ Characteristics* $^{*}$} & \multicolumn{2}{|c|}{ Cases } & \multicolumn{2}{|c|}{ Controls } & \multirow{2}{*}{$\begin{array}{c}\text { Crude OR } \\
(95 \% \mathrm{Cl})\end{array}$} & \multirow{2}{*}{$\begin{array}{l}\text { Adjusted OR } \\
\quad(95 \% \mathrm{Cl})\end{array}$} \\
\hline & $\mathbf{N}$ & $\%$ & $\mathbf{N}$ & $\%$ & & \\
\hline \multicolumn{7}{|l|}{ Method of water disinfection } \\
\hline None & 30 & 77 & 9 & 15 & $21.7(7.6-62.1)$ & $18.2(4.8-68.8)$ \\
\hline Chlorination or boiling & 8 & 21 & 52 & 85 & & \\
\hline \multicolumn{7}{|l|}{ Toilet facility } \\
\hline No toilet & 7 & 18 & 2 & 3 & $6.4(1.3-32.9)$ & $2.4(0.25-22.6)$ \\
\hline At least one & 31 & 79 & 57 & 93 & & \\
\hline \multicolumn{7}{|l|}{ Water source } \\
\hline Deep well or spring & 8 & 21 & 3 & 5 & $5.0(1.2-20.1)$ & $0.6(0.14-2.67)$ \\
\hline Other sources & 31 & 79 & 58 & 95 & & \\
\hline \multicolumn{7}{|c|}{ Wash hands with soap before eating } \\
\hline Yes & 35 & 90 & 54 & 89 & $0.8(0.16-4.39)$ & - \\
\hline No & 4 & 10 & 5 & 8 & & \\
\hline \multicolumn{7}{|c|}{ Wash hands with soap after defecating } \\
\hline Yes & 34 & 87 & 55 & 90 & $0.4(0.004-2.6)$ & - \\
\hline No & 3 & 8 & 2 & 3 & & \\
\hline \multicolumn{7}{|l|}{ Use of spoon and fork } \\
\hline Yes & 27 & 73 & 51 & 86 & $0.4(0.1-1.2)$ & - \\
\hline No & 10 & 27 & 8 & 14 & & \\
\hline
\end{tabular}

\section{Environmental investigation}

Eight of the 22 villages used the municipal water supply which, since 2012, has been manually disinfected with chlorine granules twice-weekly. This application was not interrupted after the typhoon. However, the quantity and frequency of chlorine application was based on previous practices, and the amount used was not computed based on the approximate volume of water in the tank. There was no data on the capacity of the reservoir or the current level of water. Water testing through primary health care media was irregularly done due to lack of kits. There was no routine bacteriological or physicochemical examination of any drinking-water sources.

Several damaged main distribution pipes of the system were fixed after three to four days. However, upon inspection of the municipal water system during the outbreak investigation, breaks in the distribution pipes were found with some of these submerged in river water. The intake tanks with manual chlorinators were also inspected, and there were no chlorine granules left in the pail inside the tank. It was reported that the water system pressure was at 10 psi compared to an average of $20 \mathrm{psi}$, indicating leaks in the distribution system.
Also, after the typhoon, a family built a temporary shelter at the wall of one of the two reservoirs of the municipal water system. The family practised open defecation in the absence of a toilet facility. Rectal swabs from three of the asymptomatic children from this family were negative for bacteriological culture.

\section{DISCUSSION}

This acute gastroenteritis outbreak was most likely caused by Aeromonas hydrophila and transmitted through a contaminated water source. The fact that the same bacteria were found in the municipal water system and in cases provides strong evidence of the source of infection. The distribution of cases by village also shows that the highest attack rates were recorded in areas where their main source of drinking-water was the municipal water system; a high proportion of cases using the municipal water system reported not boiling or chlorinating their drinking-water. The case-control study showed a significant association between illness and consumption of untreated drinking-water, suggesting a waterborne source. Studies have proven that boiling or chlorinating is an effective way of disinfecting water. ${ }^{3,4}$ 
Aeromonas hydrophila is a gram-negative, facultatively anaerobic bacteria widely present in aquatic environments including drinking-water systems. ${ }^{5}$ The symptoms of aeromonas gastroenteritis are not easily distinguished from gastroenteritis of other causes., ${ }^{6,7}$ There have been several outbreaks caused by Aeromonas hydrophila mostly due to contaminated drinking-water and food. A recent outbreak involving 381 cases in a school in China identified Aeromonas hydrophila as the likely pathogen with environmental investigation, showing that the implicated vegetables were washed in polluted water from a tank close to a sewage ditch, ${ }^{8}$ similar to the suspected contamination in this study.

After the typhoon, some of the distribution pipes of the water system were broken; a drop in water pressure was also reported, indicating that not all breaks were fixed. These breaks may have been the entry point for the bacterial pathogen. Chlorine applied in the reservoir was inadequate after the typhoon, which most likely allowed the growth of the bacteria.

Aeromonas hydrophila was considered the cause of this outbreak; however, several cases tested positive for Campylobacter coli, Campylobacter jejuni and Shigella sonnei. These pathogens are more common in foodborne outbreaks, but they have also been linked to waterborne outbreaks. ${ }^{9,10}$ It is possible that these pathogens were present in the water supply, but as only $500 \mathrm{ml}$ of water was collected per water source, this may not have been enough to detect their presence. Testing larger volumes of water is required to detect Campylobacter, with $4000 \mathrm{ml}$ to $20000 \mathrm{ml}$ increasing the chance of detection. ${ }^{11}$ As these pathogens were not detected in the water, the investigation cannot rule out that these infections were due to a different source or that there was more than one outbreak occurring.

There are some limitations in this investigation, mostly due to the investigation being conducted during a disaster situation. During the case-control analysis, several questionnaires had incomplete responses, and there was a low positivity rate of testing, although this may be explained as 19 of the cases had already taken antibiotics before samples were collected. This study was not able to evaluate village-related risk factors because the choice of controls in the case-control study was matched by neighbourhood. Attack rates by village should also be interpreted with caution due to the lack of denominator stability in the post-typhoon setting.

In conclusion, this study was able to identify the likely source of the outbreak as evidenced by finding the same pathogen in both water and cases. The strong association with not chlorinating or boiling drinkingwater (mostly from the municipal water supply) provides additional evidence that this was a waterborne outbreak. This study also shows that post-disaster, areas that are less damaged and that do not require ongoing evacuation centres, can still have acute gastroenteritis outbreaks. This suggests that during disaster response, all affected areas should be monitored, not just evacuation centres and that boiling or chlorinating water should be recommended for all areas affected by the disaster, even when supply systems are repaired. As part of a disaster response, all water systems should be monitored, repaired and protected from human contact. For this outbreak, we also recommend the procurement of a chlorinator machine for the municipal water system and regular monitoring of chlorine levels.

\section{Conflicts of interest}

None declared.

\section{Funding}

This gastroenteritis outbreak investigation was funded by the Department of Health, Philippines.

\section{Acknowledgements}

We are grateful for the cooperation and support of the Center for Health and Development-Eastern Visayas, local government of Kananga, Kananga Municipal Hospital, Kananga Municipal Health Office and town residents during the field investigation. We also thank the laboratory staff of the Research Institute for Tropical Medicine for testing the samples.

\section{References}

1. Philippines: Typhoon Haiyan - Situation Report No. 29 (as of 3 January 2014). Manila, United Nations Office for the Coordination of Humanitarian Affairs, 2014 (http://reliefweb.int/ sites/reliefweb.int/files/resources/OCHAPhilippinesTyphoonHaiyan No29_02January2014.pdf, 12 December 2014). 
2. Field Health Services Information System Morbidity Report: 2012-2013. Leyte, Kananga Municipal Health Office, 2014.

3. Guidelines for drinking-water quality - Volume 1: Recommendations - Third edition incorporating first and second addenda. Geneva, World Health Organization, 2008, p. 110 (http://www.who.int/water_sanitation_health/dwq/gdwq3rev/en/, accessed 12 December 2014).

4. Clasen TF et al. Microbiological effectiveness and cost of boiling to disinfect drinking water in rural Vietnam. Environmental Science \& Technology, 2008, 42:4255-4560. doi:10.1021/es7024802 pmid:18605541

5. Igbinosa IH et al. Emerging Aeromonas species infections and their significance in public health. Scientific World Journal, 2012, 2012:625023.

6. Agger WA, McCormick JD, Gurwith MJ. Clinical and microbiological features of Aeromonas hydrophila-associated diarrhea. Journal of Clinical Microbiology, 1985, 21:909-913. pmid:4008621

7. Llopis $\mathrm{F}$ et al. Epidemiological and clinical characteristics of bacteraemia caused by Aeromonas spp. as compared with
Escherichia coli and Pseudomonas aeruginosa. Scandinavian Journal of Infectious Diseases, 2004, 36:335-341. doi:10.1080/00365540410020631 pmid:15287377

8. Zhang $Q$ et al. A foodborne outbreak of Aeromonas hydrophila in a college, Xingyi City, Guizhou, China, 2012. Western Pacific Surveillance and Response Journal, 2012, 3:39-43. doi:10.5365/wpsar.2012.3.4.018 pmid:23908938

9. Kuusi $\mathrm{M}$ et al. An outbreak of gastroenteritis from a non-chlorinated community water supply. Journal of Epidemiology and Community Health, 2004, 58:273-277. doi:10.1136/jech.2003.009928 pmid:15026434

10. Godoy P et al. Outbreak of gastroenteritis caused by Campylobacter jejuni transmitted through drinking water [in Spanish]. Medicina Clínica, 2002, 119:695-698. doi:10.1016/S00257753(02)73545-8 pmid:12459108

11. Hänninen ML et al. Detection and typing of Campylobacter jejuni and Campylobacter coli and analysis of indicator organisms in three waterborne outbreaks in Finland. Applied and EnvironmentalMicrobiology,2003,69:1391-1396.doi:10.1128/ AEM.69.3.1391-1396.2003 pmid:12620821 


\title{
Tuberculosis case notification data in Viet Nam, 2007 to 2012
}

\author{
Nguyen Viet Nhung, ${ }^{a}$ Nguyen Binh Hoa, ab Pham Huyen Khanhc and Cornelia Hennig ${ }^{c}$ \\ Correspondence to Nguyen Bin Hoa (e-mail: nguyenbinhoatb@yahoo.com).
}

Tuberculosis (TB) remains a major cause of morbidity and mortality, and Viet Nam ranks 12 among the 22 high-TB burden countries. This study analyses surveillance data of the National Tuberculosis Control Programme in Viet Nam for the six-year period 2007 to 2012. During the study period, 598877 TB cases (all forms) were notified, and 313225 $(52.3 \%)$ were new smear-positive cases. The case notification rate of new smear-positive cases was decreased, from 65 per 100000 population in 2007 to 57 per 100000 population in 2012; this decrease was observed for males and females in all age groups except males aged 0-14 and females aged 15-24 years. The male-to-female ratio of new smear-positive TB cases increased from 2.85 in 2007 to 3.02 in 2012 . The average annual cure rate of new smear-positive cases was $90.3 \%$. The high male-to-female ratio for new smear-positive TB cases in this notification data was lower than that from the 2007 TB prevalence survey in Viet Nam, suggesting a lower case detection for males. The decrease in new smear-positive case notification rates may reflect a decline in TB incidence in Viet Nam as several programmatic improvements have been made, although further research is required to increase case detection among young males and children.

$\mathrm{V}$ iet Nam has approximately 90 million inhabitants in 63 provinces, 700 districts and 11145 communes. ${ }^{1}$ Tuberculosis (TB) remains a major cause of morbidity and mortality, with Viet Nam ranking 12 among the 22 high-TB burden countries. ${ }^{2}$ In 2012, the estimated TB incidence (including HIVpositive patients) was 147 per 100000 population; prevalence was 218 per 100000 population; and mortality (excluding HIV) was 20 per 100000 population. TB incidence among HIV-positive cases was estimated to be 10 per 100000 population. $^{2}$

The Viet Nam National Tuberculosis Control Programme (NTP) commenced in 1986, with the directly observed treatment, short-course (DOTS) strategy initiated in 1992, reaching $100 \%$ DOTS coverage by $2000 .{ }^{3}$ The Viet Nam TB Information Management Electronic System (VITIMES) was established in 2009 and is a web-based system designed to collect patientbased data on patients screened for TB, on notified TB cases and on the treatment outcomes of TB patients. Data sources comprise paper reports, existing registers and laboratory forms. VITIMES was introduced in two phases: Phase I at the provincial level with aggregated data being entered from quarterly district paper reports and Phase II at the district level where individual patient information is entered. Phase I was implemented by all
63 provinces in 2010; Phase II coverage is expected nationwide by 2015 .

The objectives of this report are to assess case notification rates of all TB and new smear-positive TB cases over time and to provide a summary by age, sex and treatment outcomes in Viet Nam from 2007 to 2012.

\section{METHODS}

This descriptive study used TB surveillance data routinely collected by NTP from 2007 to 2012, disaggregated by age and sex, as well as treatment outcome reports from aggregate district-level reports entered into VTIMES.

Data were exported to EpiData and analysed using Excel and EpiData (Version 2.2, http://www.epidata. $\mathrm{dk}$ ). Differences in proportions were assessed using the chi-square test, and differences in case notification rates were assessed using poison regression testing. Point estimates are shown with 95\% confidence intervals (Cl) for proportions.

The TB patient categories followed those described in the Revised international definitions in tuberculosis control, ${ }^{4}$ and the positivity rate of presumptive TB cases

\footnotetext{
a National Tuberculosis Programme Vietnam, Ha Noi, Viet Nam.

b Centre for Operational Research, International Union Against Tuberculosis and Lung Disease, Paris, France.

Office of the WHO Representative in Viet Nam, Ha Noi, Viet Nam. Submitted: 27 April 2014; Published: 9 February 2015

doi: 10.5365/wpsar.2014.5.2.005
} 
Figure 1. Number of presumptive TB patients examined by sputum microscopy; number of sputum smear-positive cases detected and positivity rate, ${ }^{*}$ Viet Nam, 2007-2012

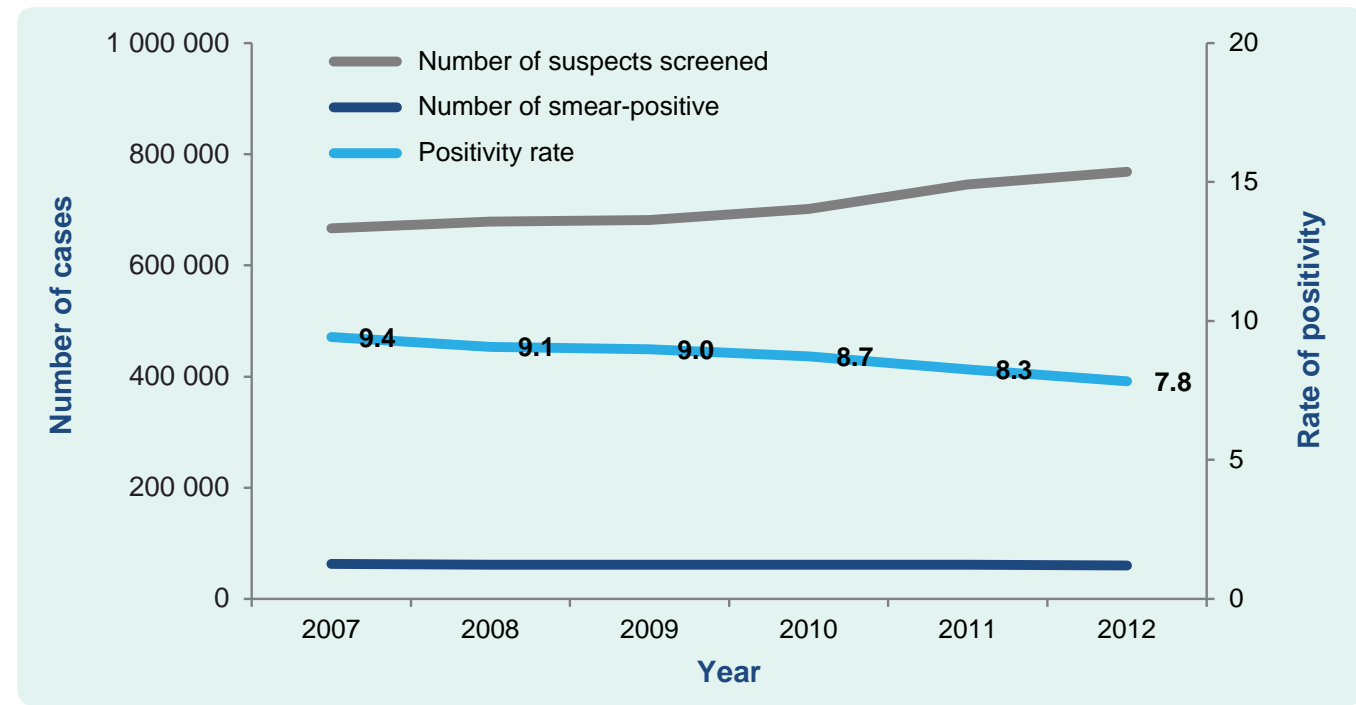

* Positivity rate: the number of sputum smear-positive cases divided by the total number of presumptive tuberculosis cases screened by microscopy.

was the number of sputum-positive cases divided by the total number of presumptive TB cases screened by microscopy.

\section{RESULTS}

\section{TB cases}

Between 2007 and 2012, a total of 4243630 presumptive TB cases were examined by sputum smear microscopy, with 368594 (8.7\%) being smear-positive. The number screened increased from 666947 in 2007 to 768449 in 2012, with the positivity rate significantly decreased from $9.4 \%$ in 2007 to $7.8 \%$ in $2012(P<0.001$, Figure 1$)$.

There were 598877 TB cases (all forms) notified: 313225 (52.3\%) were new smear-positive, 120769 (20.2\%) were smear-negative, 115450 (19.3\%) were extrapulmonary, and 49433 (8.3\%) were cases previously treated. The proportion of new smear-positive cases decreased from $55.4 \%$ in 2007 to $49.1 \%$ in $2012(P<0.001)$; in contrast, the proportion of previously treated cases increased from $7.8 \%$ in 2007 to $8.7 \%$ in $2012(P<0.001)$, and the proportion of new smear-negative cases increased from $17.8 \%$ in 2007 to $22.4 \%$ in $2012(P<0.001$, Figure 2$)$. While the annual case notification rate of TB (all forms) remained stable over the study period (range 114 to 117 per
100000 population, $P=0.749$ ), the case notification rate of new smear-positive TB cases significantly declined, from 65 to 57 per 100000 population between 2007 and 2012, respectively $(P<0.001$, Figure 3$)$.

\section{New smear-positive cases}

The average annual case notification rate of new smear-positive TB cases for the period 2007 to 2012 was 60.4 per 100000 population (95\% Cl: 60.2-60.6). Age-specific case notification rates increased with increasing age group, from 0.5 per 100000 population ( $95 \% \mathrm{Cl}: 0.5-0.6$ ) for the $0-14$ year age group to 162.9 per 100000 population (95\% Cl: 161.6-164.2) for those aged 65 years and above (Table 1).

There was a significant decline in the smear-positive notification rate between 2007 and 2012 for all age groups ( $p<0.05$ for all). Sex-specific average annual case notification rates also declined between 2007 to 2012 for both males and females in each of the five age groups except for males aged $0-14$ and females aged 15-24 ( $P=0.324$ and $P=0.353$, respectively). In contrast, there was a significant increase in the smear-positive notification rate of female aged $0-14$ $(P<0.001$, Figure 4).

The total male-to-female ratio of new smear-positive TB cases increased from 2.85 in 2007 to 3.02 in 2012. 
Figure 2. Proportion of TB cases (all forms) by case classification and year, Viet Nam, 2007-2012

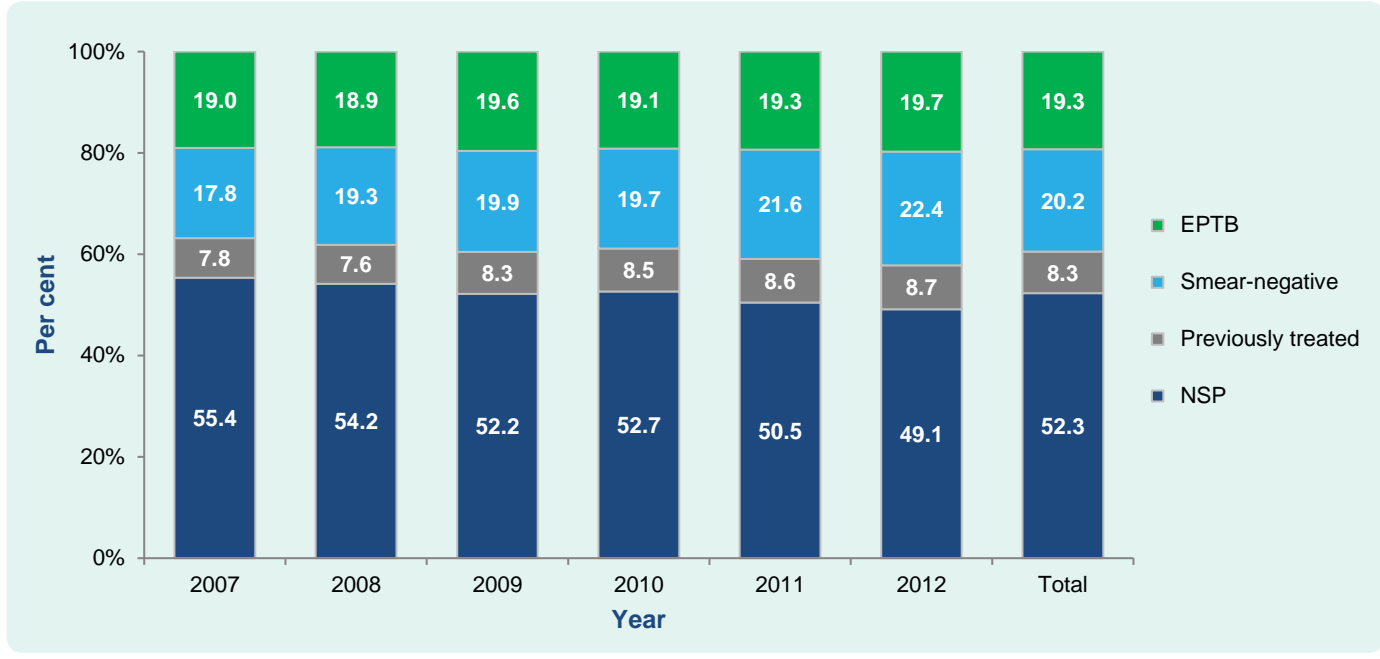

EPTB, extrapulmonary tuberculosis; NSP, new smear-positive tuberculosis.

Figure 3. Case notification rate of TB (all forms) and new smear-positive TB cases per 100000 population, Viet Nam, 2007-2012

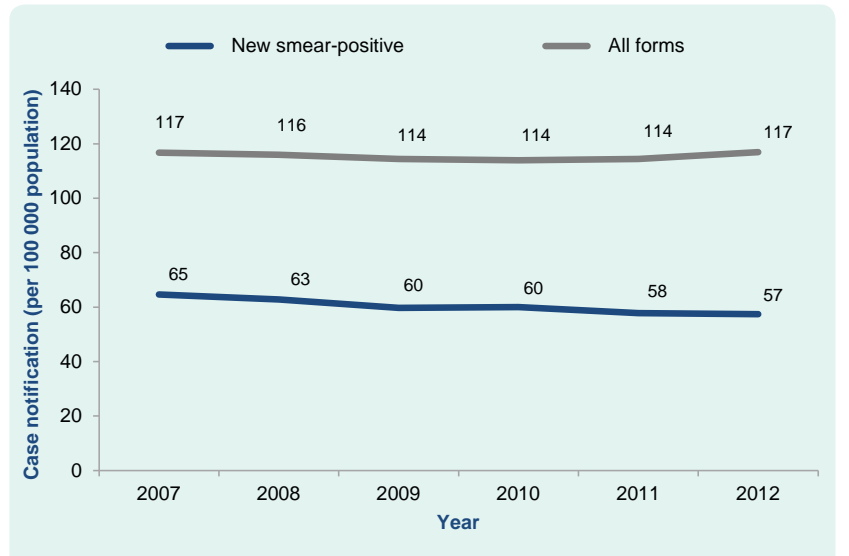

An observation: over the study period, age group-specific male-to-female ratios seemed to increase for the four age groups 35-44, 45-54, 55-64 and 65 year and above age groups, otherwise this ratios seemed to be constant or decreasing (Table 2).

The 2012 case notification rate of new smear-positive TB by province showed that rates are generally higher in the southern provinces compared with the northern provinces (Figure 5).

Although the number of children with smear-positive TB increased from 84 in 2008 to 142 cases in 2012, the proportion of children aged 0-14 among new smear-positive TB cases was low (0.2\%) during the period 2007 to 2012.

Table 1. Average annual case notification rate of new smear-positive TB cases by sex and age group, Viet Nam, 2007-2012

\begin{tabular}{|c|c|c|c|c|c|c|c|c|c|}
\hline \multirow{3}{*}{$\begin{array}{l}\begin{array}{c}\text { Age group } \\
\text { (years) }\end{array} \\
0-14\end{array}$} & \multicolumn{3}{|c|}{ Total } & \multicolumn{3}{|c|}{ Male } & \multicolumn{3}{|c|}{ Female } \\
\hline & \multirow{2}{*}{$\begin{array}{c}\begin{array}{c}\text { Notification } \\
\text { per } 100000 \\
\text { population }\end{array} \\
0.5\end{array}$} & \multicolumn{2}{|c|}{$\begin{array}{l}95 \% \text { confidence } \\
\text { interval }\end{array}$} & \multirow{2}{*}{$\begin{array}{c}\begin{array}{c}\text { Notification } \\
\text { per } 100000 \\
\text { population }\end{array} \\
0.5\end{array}$} & \multicolumn{2}{|c|}{$\begin{array}{l}95 \% \text { confidence } \\
\text { interval }\end{array}$} & \multirow{2}{*}{$\begin{array}{c}\begin{array}{c}\text { Notification } \\
\text { per } 100000 \\
\text { population }\end{array} \\
0.6\end{array}$} & \multicolumn{2}{|c|}{$\begin{array}{c}95 \% \text { confidence } \\
\text { interval }\end{array}$} \\
\hline & & 0.5 & 0.6 & & 0.4 & 0.5 & & 0.5 & 0.6 \\
\hline $15-24$ & 32.4 & 32.1 & 32.8 & 40.2 & 39.7 & 40.8 & 24.4 & 23.9 & 24.8 \\
\hline $25-34$ & 67.3 & 66.8 & 67.9 & 101.2 & 100.2 & 102.2 & 34.0 & 33.5 & 34.6 \\
\hline $35-44$ & 75.0 & 74.4 & 75.6 & 123.6 & 122.5 & 124.7 & 26.8 & 26.2 & 27.3 \\
\hline $45-54$ & 97.6 & 96.9 & 98.4 & 165.0 & 163.6 & 166.5 & 34.9 & 34.2 & 35.5 \\
\hline $55-64$ & 133.7 & 132.5 & 134.9 & 218.9 & 216.6 & 221.3 & 61.4 & 60.3 & 62.6 \\
\hline 65 and above & 162.9 & 161.6 & 164.2 & 260.3 & 257.7 & 263.0 & 97.9 & 96.6 & 99.3 \\
\hline Total & 60.4 & 60.2 & 60.6 & 90.7 & 90.3 & 91.1 & 30.8 & 30.5 & 31.0 \\
\hline
\end{tabular}


Figure 4. Case notification rate of new smear-positive TB cases by sex, age group and year, Viet Nam, 2007-2012*
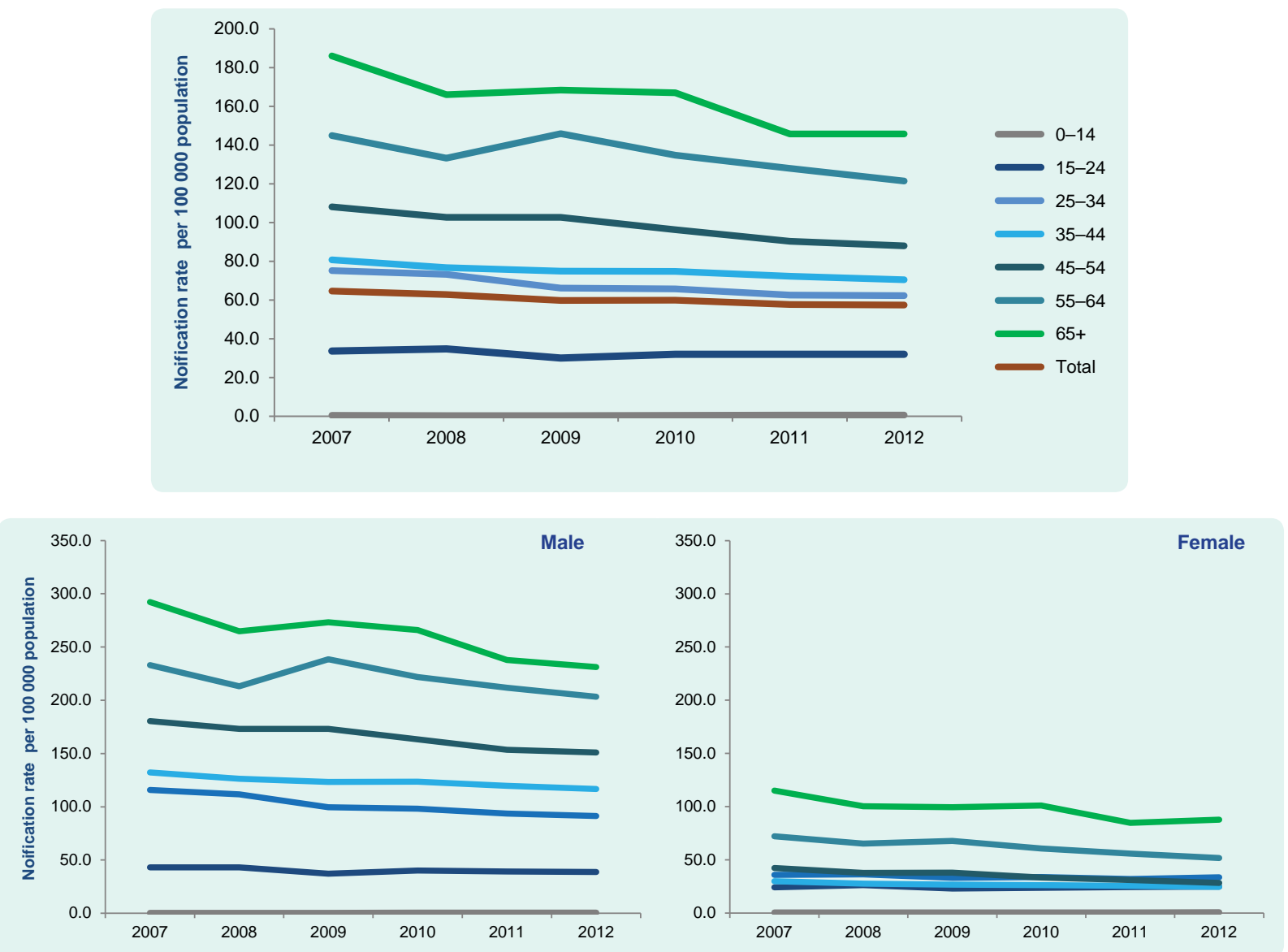

* $P$-value for all age groups was $<0.05$, except for the male $0-14$ age group $(p=0.324)$ and the female $15-24$ age group $(p=0.353)$.

Treatment outcome of new smear-positive cases

The cure rate of new smear-positive cases was maintained at high levels (above 85\%) for all years in the study period; although this increased slightly from $89.8 \%$ in 2007 to $91.1 \%$ in 2012 , the difference was not statistically significant $(P=0.089$, Figure 6$)$. The average annual cure rate for six years was $90.3 \%$. The six-year annual averages for the other treatment outcomes were: treatment completed at $2.0 \%$, death at $3.0 \%$, treatment failure at $1.1 \%$, treatment after default at $1.7 \%$ and transferred out at $1.9 \%$.

Table 2. Male-to-female ratio of new smear-positive TB cases by age group and year, Viet Nam, 2007-2012

\begin{tabular}{cccccccc}
\hline Age group (years) & $\mathbf{2 0 0 7}$ & $\mathbf{2 0 0 8}$ & $\mathbf{2 0 0 9}$ & $\mathbf{2 0 1 0}$ & $\mathbf{2 0 1 1}$ & $\mathbf{2 0 1 2}$ & Total \\
\hline $0-14$ & 0.8 & 0.7 & 0.8 & 1.0 & 0.9 & 0.6 & 0.8 \\
$15-24$ & 1.8 & 1.6 & 1.6 & 1.7 & 1.6 & 1.6 & 1.6 \\
$25-34$ & 3.2 & 3.1 & 3.0 & 2.9 & 2.9 & 2.7 & 3.0 \\
$35-44$ & 4.4 & 4.6 & 4.6 & 4.7 & 4.7 & 4.8 & 4.6 \\
$45-54$ & 4.3 & 4.6 & 4.6 & 4.9 & 5.0 & 5.3 & 4.7 \\
$55-64$ & 3.2 & 3.3 & 3.5 & 3.7 & 3.8 & 3.9 & 3.6 \\
65 and above & 2.5 & 2.6 & 2.7 & 2.6 & 2.8 & 2.6 & 2.7 \\
Total & 2.9 & 2.9 & 2.9 & 3.0 & 3.1 & 3.0 & 3.0 \\
\hline
\end{tabular}


Figure 5. Case notification rates of new smear-positive TB cases by province, Viet Nam, 2012

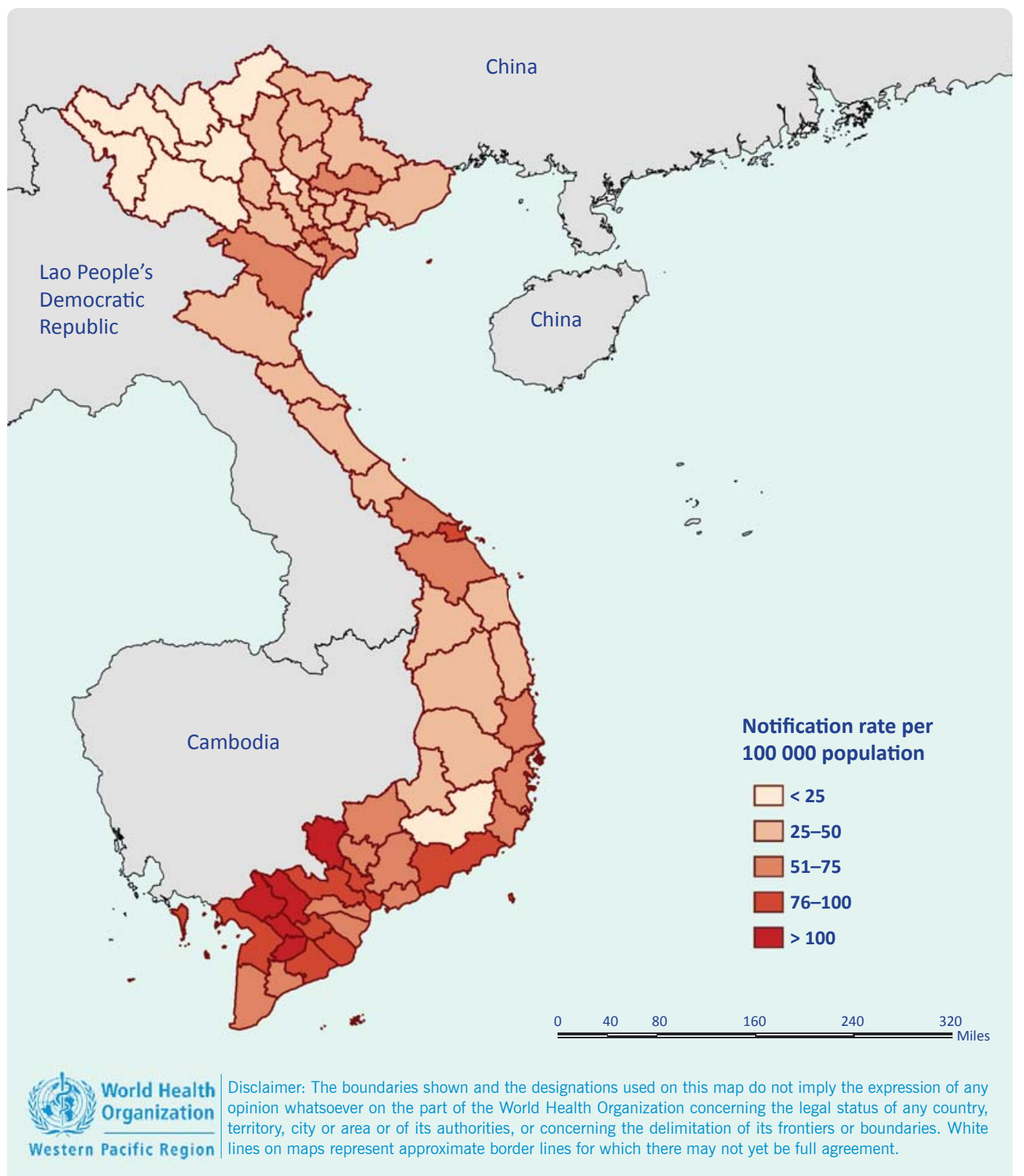

\section{DISCUSSION}

The data from the NTP in Viet Nam showed a stable case notification rate of all forms of TB for the years 2007 to 2012, ranging from 114 to 117 per 100000 population, yet a significant decrease for new smear-positive TB cases, dropping from 65 to 57 per 100000 population from 2007 to 2012, respectively. Whether this decrease in the new smear-positive case notification rate reflects an actual decrease in infectious cases of TB is an important programmatic question. The number of presumptive TB cases who had a sputum smear examination increased over the study period, with the positivity rate significantly decreasing from $9.4 \%$ to $7.8 \%$. During the same period the Viet Nam NTP focused on improving TB case finding by engaging private and public facilities outside the NTP; improving chest X-ray (CXR) availability and quality; introducing activities to reach presumptive TB cases in remote areas; and through advocacy, communication and social mobilization activities. The Viet Nam NTP also expanded TB activities in prisons, with the number of TB units in prisons expanding from 24 in 2007 to 51 in 2012. In addition, the quality of sputum examination has improved each year and the proportion of HIV coinfection among TB patients was reduced from 8\% 
Figure 6. Proportion of new smear-positive TB cases by treatment outcome and year, Viet Nam, 2007-2012

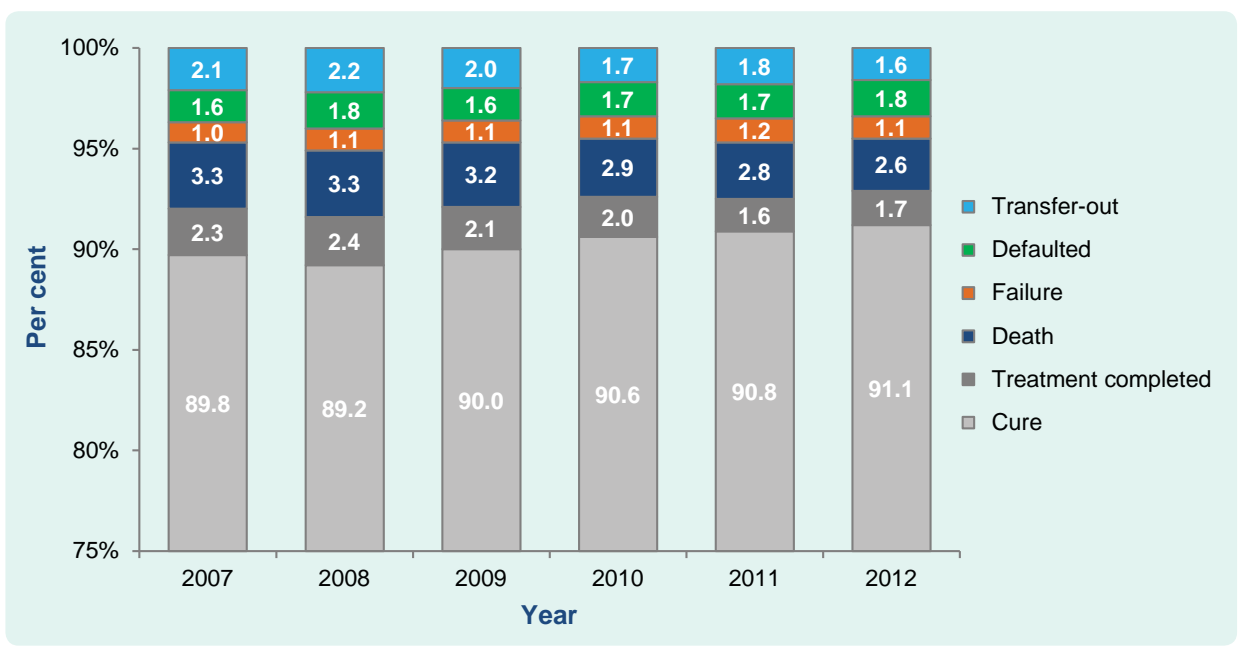

in 2007 to $7 \%$ in $2012 .^{2}$ Case reporting has also been improved by introducing electronic reporting systems. The latest World Health Organization (WHO) estimates for TB (January 2013) also suggest that the burden of TB in Viet Nam is estimated to decline in incidence by $2.6 \%$ per year, and in prevalence and mortality by $4 \%$ and $5 \%$ per year, respectively. ${ }^{5}$

In our study, the smear-positive TB case notification rates in Viet Nam increased with age; this trend has also been reported in many other countries., The first national tuberculosis prevalence survey in Viet Nam (2006-2007) also showed a strong and statistically significant correlation between increasing age and the prevalence of CXR abnormalities, persistent productive cough and bacteriologically confirmed TB. ${ }^{6}$ In our study, more than half of new smear-positive cases notified in 2012 (54\%) were aged 45 years and above. This is in contrast to the proportion of persons in the population aged 45 years and above, which was only $28 \% .^{7}$

Demographic changes in population over time may affect trends in TB notification rates. ${ }^{8}$ Viet Nam population data have shown an increase in the proportion of persons aged 45 and above from 25\% in 2007 to $28 \%$ in $2012 .{ }^{7}$ This increase in the older population may cause a lower-than-expected decline in the overall case notification rates of TB as older people have higher TB notification rates, and younger persons have lower TB notification rates but make up a smaller proportion of the population.
To control for this demographic factor, we also analysed the notification rates of new smear-positive TB by age group over time. There was a significant decline in the smear-positive notification rate between 2007 and 2012 for all age groups except for males aged 0-14 years and females aged 15-24 years. This finding differs from that observed for notified cases in the period 1997 to 2004, when TB notification decreased in all age groups except among young men in the $15-35$ year age group. ${ }^{9}$

In this study, the proportion of children among new smear-positive TB cases was less than the estimated proportion of TB in children in low-income countries. ${ }^{10}$ This low proportion may be due to there being less smear-positive TB occurring in children or because of underreporting of childhood TB to the NTP. As a result of this, since 2012, the NTP has been intensifying efforts to diagnose and notify childhood TB by strengthening TB case finding and screening of children in close contact with an adult TB case.

We observed that the male-to-female ratio in notified smear-positive cases increased from 2.85:1 in 2007 to $3.02: 1$ in 2012 . This ratio is much higher than the male-to-female ratio among notified new smear-positive cases in the 22 high-burden countries (1.9:1), in the WHO Western Pacific Region (2.4:1) and worldwide $(1.9: 1){ }^{2}$ The 2006 to 2007 national TB prevalence survey in Viet Nam reported a maleto-female ratio of $4.8: 1$ for new smear-positive cases and 4.6:1 in bacteriologically confirmed $\mathrm{TB}^{6}{ }^{6}$ These 
data support the hypothesis that the difference in TB notification rates in males and females in Viet $\mathrm{Nam}$ reflects a true difference in disease occurrence rather than a difference in access to diagnosis and treatment as previous studies have suggested. ${ }^{11}$ The higher maleto-female ratio in the prevalence survey compared to the notification data suggests lower case detection in males compared with females. A possible explanation for this preponderance of males might be the large difference in the proportion of men and women who smoke. ${ }^{12}$ In 2008, WHO reported that in Viet Nam, $2 \%$ of women are current smokers compared to $44 \%$ of men, and $34 \%$ of men smoke daily. ${ }^{13}$ Other possible explanations for the high male-to-female ratio may be the higher HIV prevalence among males compared to females ${ }^{14}$ and higher alcohol consumption among men. ${ }^{15}$ More research to investigate potential differences in the epidemiology of TB between males and females is needed as well as improved case finding among men.

This analysis was based on notification data reported to VITIMES. For the results to truly reflect the TB epidemiology in Viet Nam, they are dependent on not only the quality of the surveillance system but also the capacity of the NTP to detect and treat all TB cases in the community. The TB surveillance system in Viet Nam has been assessed as a well-functioning system with many strengths but also several gaps. ${ }^{5}$ First, the Viet Nam NTP does not yet collect information on sex and age for all TB cases; this is currently only available for new smear-positive TB cases. Second, not all care providers in the public and private sectors report to the NTP, resulting in notification gaps, including for childhood TB, which suggests an underreporting of cases. Third, as VITIMES is not yet implemented in all districts providing individual case-based data, our data analysis was based on aggregated reports from district levels which restrict analysis. The TB information system should be further strengthened to improve data quality and coverage in non-NTP facilities.

Despite these limitations, this report has shown an increase in TB testing in Viet Nam coupled with a decrease in notification of new smear-positive cases between 2007 and 2012. The case notification rate of smear-positive TB increased significantly with age, and a male-to-female ratio lower than the 2007 prevalence survey suggests lower case detection in males compared with females. These results suggest that the NTP activities to increase case detection have been effective, although further research is required to increase case detection among young males and children.

\section{Conflicts of interest}

None declared.

\section{Funding}

None.

\section{Acknowledgements}

The authors would like to thank the staff of the Viet Nam National Tuberculosis Control Programme, especially the data management team.

\section{References}

1. General Statistics Office. Ha Noi, Government of Viet Nam, 2014 (http://www.gso.gov.vn/default.aspx?tabid=386\&idmid=3\&Item $I D=14154$, accessed 18 December 2014).

2. Global tuberculosis report 2013. Geneva, World Health Organization, $2013 \quad$ (http://apps.who.int/iris/ bitstream/10665/91355/1/9789241564656_eng.pdf, accessed 18 December 2014).

3. Huong NT et al. Establishment and development of the National Tuberculosis Control Programme in Viet Nam. International Journal of Tuberculosis and Lung Disease, 2005, 9:151-156. pmid: 15732733

4. World Health Organization, International Union Against Tuberculosis and Lung Disease, Royal Netherlands Tuberculosis Association. Revised international definitions in tuberculosis control. International Journal of Tuberculosis and Lung Disease, 2001, 5:213-215. pmid:11326818

5. Bloss $\mathrm{E}$ et al. Evaluation of TB surveillance in Viet Nam. Mission report, 14-18 January 2013.

6. Hoa NB et al. National survey of tuberculosis prevalence in Viet Nam. Bulletin of the World Health Organization, 2010, 88:273-280. doi:10.2471/BLT.09.067801 pmid: 20431791

7. Population pyramid, Viet Nam 2012. Belgium, Cooperation Exchange Training, 2014 (http://www.coopami.org/en/countries/ other_countries/vietnam/country_description/index.htm, accessed 18 Décember 2014).

8. Hiatt T, Nishikiori N. Epidemiology and control of tuberculosis in the Western Pacific Region: analysis of 2012 case notification data. Western Pacific Surveillance and Response Journal, 2014, 5:2534. doi:10.5365/wpsar.2014.5.1.013 pmid:24734214

9. Vree $\mathrm{M}$ et al. Tuberculosis trends, Vietnam. Emerging Infectious Diseases, 2007, 13:796-797. doi:10.3201/eid1305.060904 pmid: 18044050

10. Marais BJ et al. The burden of childhood tuberculosis and the accuracy of community-based surveillance data. International Journal of Tuberculosis and Lung Disease, 2006, 10:259-263. pmid: 16562704

11. Rieder HL. Epidemiologic basis of tuberculosis control. Paris, International Union Against Tuberculosis and Lung Disease, 1999. 
12. Holmes CB, Hausler H, Nunn P. A review of sex differences in the epidemiology of tuberculosis. International Journal of Tuberculosis and Lung Disease, 1998, 2:96-104. pmid:9562118

13. WHO report on the global tobacco epidemic, 2008. Geneva, World Health Organization, 2008 (http://www.who. int/tobacco/mpower/mpower_report_full_2008.pdf, 18 December 2014).
14. Thanh $\mathrm{DH}$ et al. HIV infection among tuberculosis patients in Viet Nam: prevalence and impact on tuberculosis notification rates. International Journal of Tuberculosis and Lung Disease, 2010, 14:986-993. pmid:20626943

15. Global status report on alcohol and health 2011. Geneva, World Health Organization, 2011 (http://www.who.int/substance_abuse/ publications/global alcohol report/msbgsruprofiles.pdf, accessed

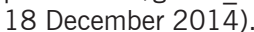




\title{
Tuberculosis case-finding in Cambodia: analysis of case notification data, 2000 to 2013
}

\author{
Fukushi Morishita, ${ }^{a}$ Valérie Burrus Furphy, ${ }^{b}$ Miwako Kobayashi, ${ }^{b}$ Nobuyuki Nishikiori, ${ }^{a}$ Mao Tan Eang ${ }^{c}$ and \\ Rajendra-Prasad Yadav \\ Correspondence to Fukushi Morishita (e-mail: morishita.fukushi@gmail.com).
}

The routine tuberculosis (TB) surveillance system in Cambodia has been strengthened under the National TB Programme (NTP). This paper provides an overview of the TB surveillance data for Cambodia at the national level for the period 2000 to 2013 and at the subnational level for 2013.

The proportion of the total population that were screened for TB rose from $0.4 \%$ in 2001 to $1.1 \%$ in 2013 , while the smear-positivity rate decreased from $28.9 \%$ to $8.1 \%$ in the same period. The total number of notified TB cases increased steadily from 2000; this has stabilized in recent years with 39055 cases notified in 2013. The proportion of all TB cases that were smear-positive decreased from $78 \%$ in 2000 to $36 \%$ in 2013 . Case notification rates (CNRs) for all forms of TB and new smear-positive TB in 2013 were 261 and 94 per 100000 population, respectively. Higher CNRs were found in the north-west and south-east parts of the country and were higher for males especially in older age groups.

The increase in TB screening, decline in the smear-positive rate and decline in notified smear-positive TB cases likely reflect a long-term positive impact of the NTP. A negative correlation between the proportion of the population screened and the smear-positivity rate at the subnational level helped identify where to find undiagnosed cases. Subnational differences in case notification of the elderly and in children provide more specific targets for case-finding and further encourage strategic resource allocation.

S urveillance was one of the five core components in the original World Health Organization (WHO) Framework for Effective Tuberculosis Control (the DOTS Strategy). ${ }^{1}$ Routine surveillance data can be used to assess disease burden and epidemiological trends as well as to identify underserved populations and potential outbreaks. ${ }^{2}$ Its effective use is essential for informed programmatic decision-making. ${ }^{3}$ Tuberculosis (TB) programmes establish and maintain a recording and reporting (R\&R) system with regular communication between the central and peripheral levels of the health system as its surveillance system. . $^{1,2,4}$

Since the relaunch of the National TB Programme (NTP) in 1994, Cambodia has strengthened its routine TB surveillance system. ${ }^{5,6}$ As in many other countries, Cambodia's NTP has adopted a case-based registry and cohort monitoring of treatment outcomes using standardized tools in line with the WHO-recommended
R\&R framework. ${ }^{4}$ Recognizing the importance of evidence-based policy and practice in public health, Cambodia's NTP has invested substantial efforts and resources in quarterly data collection and the periodic analysis of routine surveillance data and its documentation at the national level while intensifying subnational data analysis. ${ }^{5}$

This paper provides an overview of TB case-finding in Cambodia at the national level for 2000 to 2013 and the subnational level for 2013.

\section{METHODS}

We performed a retrospective descriptive analysis of TB case-finding in Cambodia using routine TB surveillance data sourced from the national TB database. The data were analysed by disease category, demographic variables and geographical areas.

\footnotetext{
World Health Organization Regional Office for the Western Pacific, Manila, Philippines.

Office of the WHO Representative in Cambodia, Phnom Penh, Cambodia.

National Center for Tuberculosis and Leprosy Control, Ministry of Health, Phnom Penh, Cambodia.

Submitted: 18 November 2014; Published: 26 February 2015

doi: 10.5365/wpsar.2014.5.4.005
} 
Figure 1. Population-screening rate and smear-positivity rate for TB, Cambodia, 2001-2013

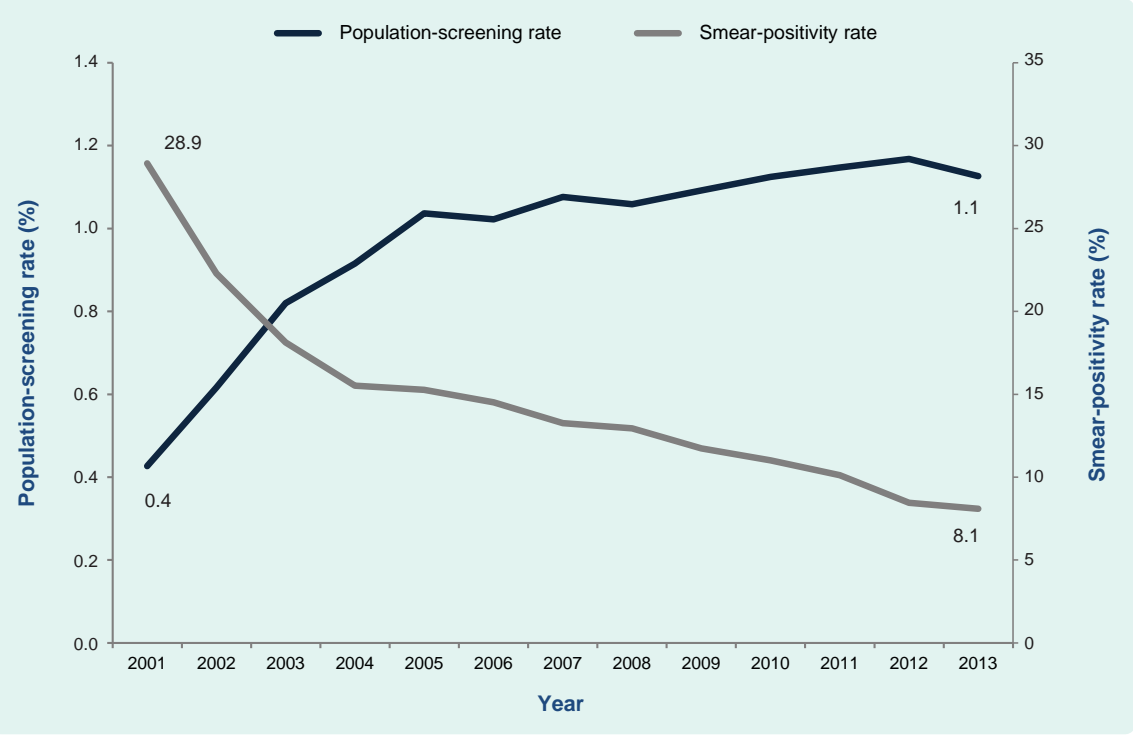

Note: Population-screening rate: the number of suspected TB cases examined by smear microscopy divided by the total population in each year. Smear-positivity rate: the number of positive slides divided by the total number of slides examined for TB diagnosis.

In the Cambodian health system, the basic organizational and reporting unit is an operational district (OD), which also serves as a basic management unit of the TB programme. Each OD provides services through health centres and district referral hospitals to an average population of $180000 .^{7}$

The standardized data collection of the TB surveillance system operates in a stepwise approach in accordance with the WHO-recommended R\&R framework. ${ }^{4,8}$ A TB case register comprising a line listing of individual patient records is kept at the health centre level. This is regularly consolidated into an OD TB register that references all notified cases within the OD. Although provinces play a certain role within the reporting line, the OD TB register is the basis of a quarterly TB report from which data are entered into several national databases including case notification data by age/sex and patient/ disease category, treatment outcomes and laboratory data.

The disaggregated national databases, which are maintained in Excel, were consolidated for analysis and producing tables and figures. QGIS 2.2 software (GNU General Public License, 2013) was used to produce maps depicting geographical distribution of case notification rates (CNRs).

We defined a population-screening rate as the number of suspected TB cases examined by smear microscopy divided by the total population in each year.
The population-screening rate is used as a proxy to assess case-finding efforts. The smear-positivity rate was defined as the number of positive slides divided by the total number of slides examined for TB diagnosis. The smear-positivity rate is also a useful indicator to assess the impact of the programme in reducing TB prevalence. ${ }^{9}$

National population data were sourced from the WHO global TB database; subnational population data were sourced from the Cambodian Health Management Information System. Age-specific and province-specific population data were sourced from the projected population in the Census. Pearson's correlation coefficient was used to determine any correlation between smearpositivity rate and population-screening rate, adult and childhood TB notifications and elderly and non-elderly TB notifications.

Ethics clearance was not required as this report used routinely available data and no personal identifying information was collected.

\section{RESULTS}

\section{TB screening and smear-positivity rate}

The population-screening rate for TB at the national level rose from $0.4 \%$ in 2001 to $1.1 \%$ in 2013 , while the smear-positivity rate decreased from $28.9 \%$ to $8.1 \%$ over the same period (Figure 1). By province, the population-screening rate ranged from $0.3 \%$ in Kep 
Figure 2. Scatterplot of population-screening rate and smear-positivity rate by province, Cambodia, 2013*

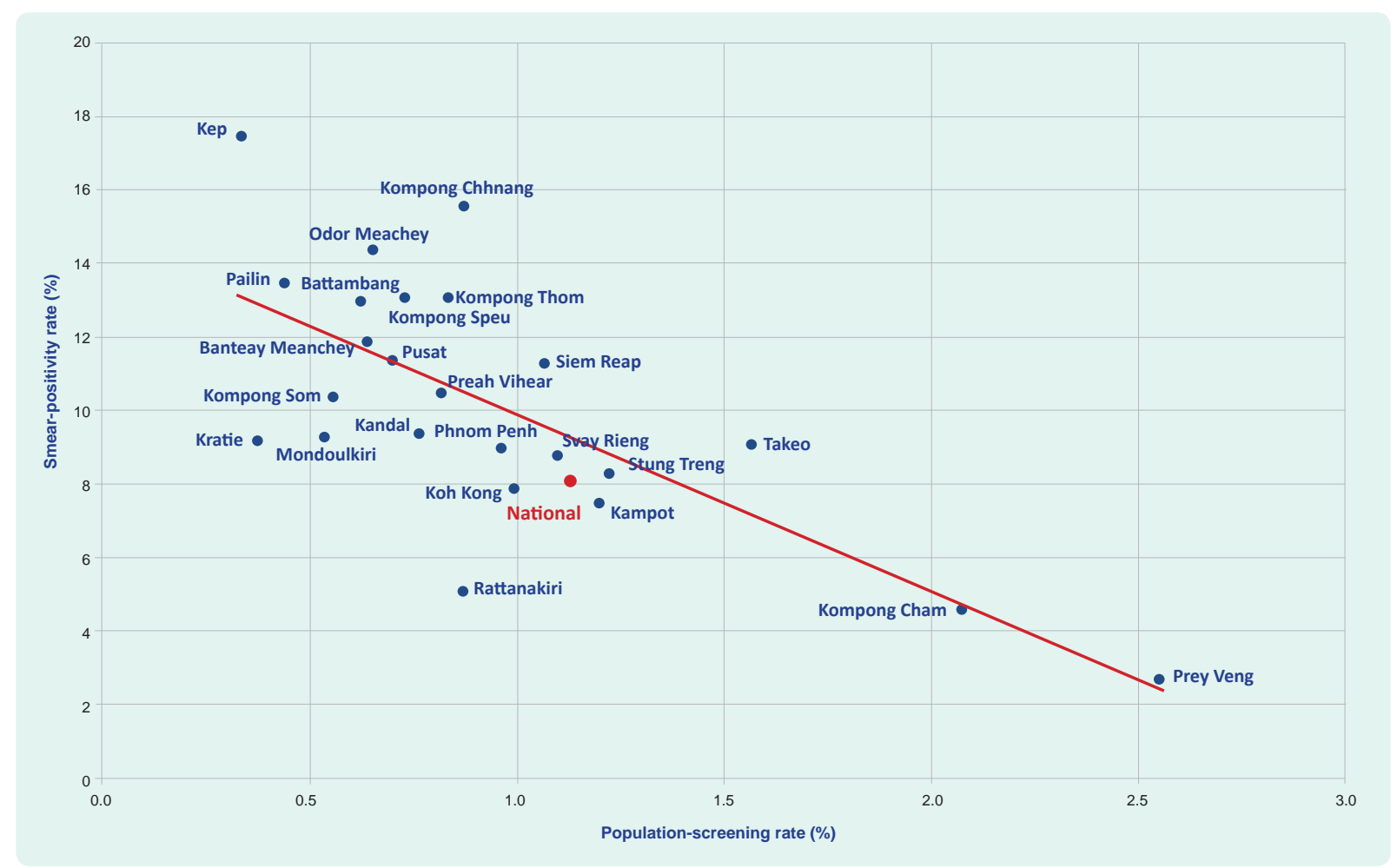

Population-screening rate $=$ the number of suspected TB cases examined by smear microscopy divided by the total population in each year. Smear-positivity rate $=$ the number of positive slides divided by the total number of slides examined for TB diagnosis.

to $2.5 \%$ in Prey Veng, whereas the smear-positivity rate ranged from $2.7 \%$ in Prey Veng to $17.5 \%$ in Kep. There was a negative correlation between these two indicators (Pearson's correlation coefficient -0.71 , $P<0.001$ ), suggesting that a higher smear-positivity rate is likely to be reported in provinces with a lower population-screening rate and vice versa (Figure 2 ).

\section{National TB case notification}

The total number of notified cases increased steadily from 19007 to 41628 between 2000 and 2010 (Figure 3a) and stabilized in recent years with 39055 cases notified in 2013. Notifications of extrapulmonary and smear-negative cases have increased consistently; since 2005, notifications of smear-positive cases have decreased (Figures $3 a$ and $3 \mathrm{~b}$ ). The proportion of new smear-positive cases of all TB notifications decreased from $78 \%$ in 2000 to $36 \%$ in 2013 (Figure 3b). In 2013, the national CNR for all forms of TB and smear-positive TB were 261 and 94 per 100000 population, respectively (Figure $3 \mathrm{c}$ ).

In every year from 2011 to 2013, the highest number of notifications of new smear-positive TB was reported in the 45-54 year age group (Figure 4). Males had higher sex-specific CNRs compared with females, especially in the older age groups; males had nearly twice the CNR rate as females in the same age group (Figure 4). The highest rate was found in males aged over 65 years in 2013 at 618 per 100000 population, more than six times higher than the total CNR for 2013 at 94 per 100000 population.

\section{Subnational TB case notification}

There are considerable differences between provinces in CNR (Figure 5). For all forms of TB, Mondolkiri had the lowest CNR at 62 per 100000 population; Svay Rieng's CNR was more than seven times higher at 477 per 100000 population. The percentage of smear-positive cases among new pulmonary cases ranged from $42.2 \%$ to $90.0 \%$ by province compared with the national figure of $60.8 \%$ (Table 1 ).

ODs with high CNRs were found in the north-west and south-east parts of the country (Figure 6); these are areas with major population concentration and movement within the country and high cross-border migration from and into Thailand and Viet Nam. 
Figure 3a. Number of TB notifications by category, Cambodia, 2000-2013

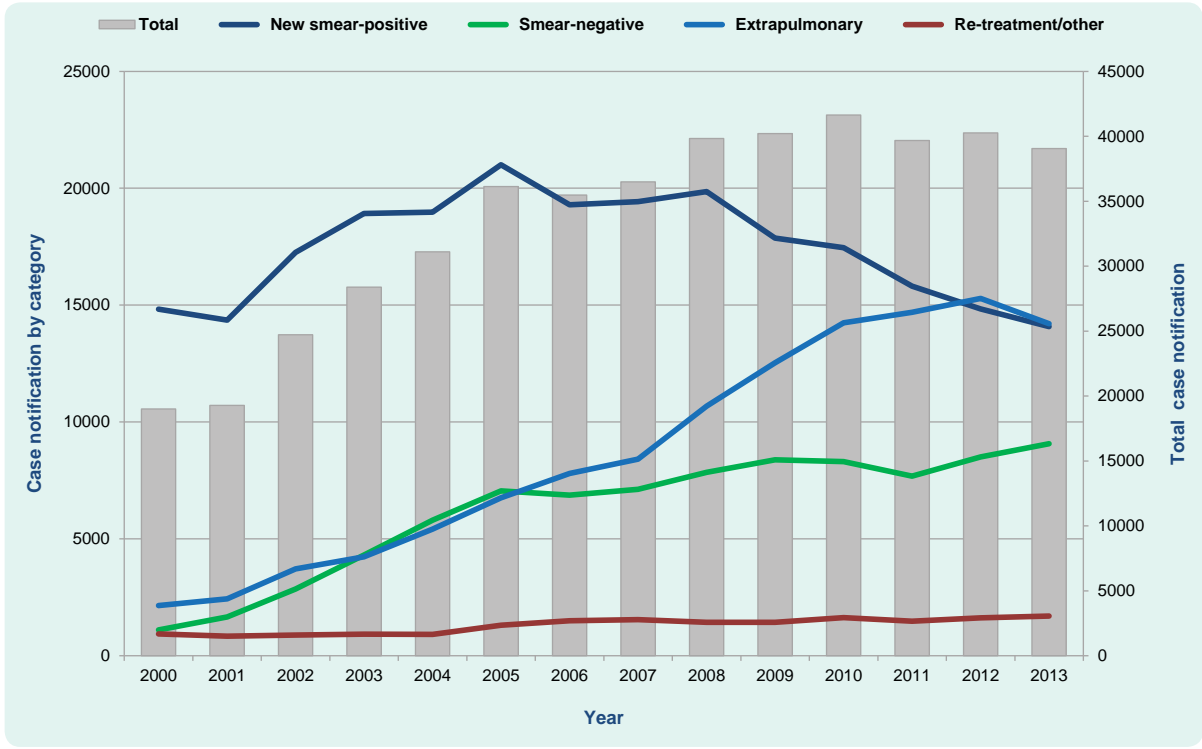

Figure 3b. Proportion of TB notifications by category, Cambodia, 2000-2013

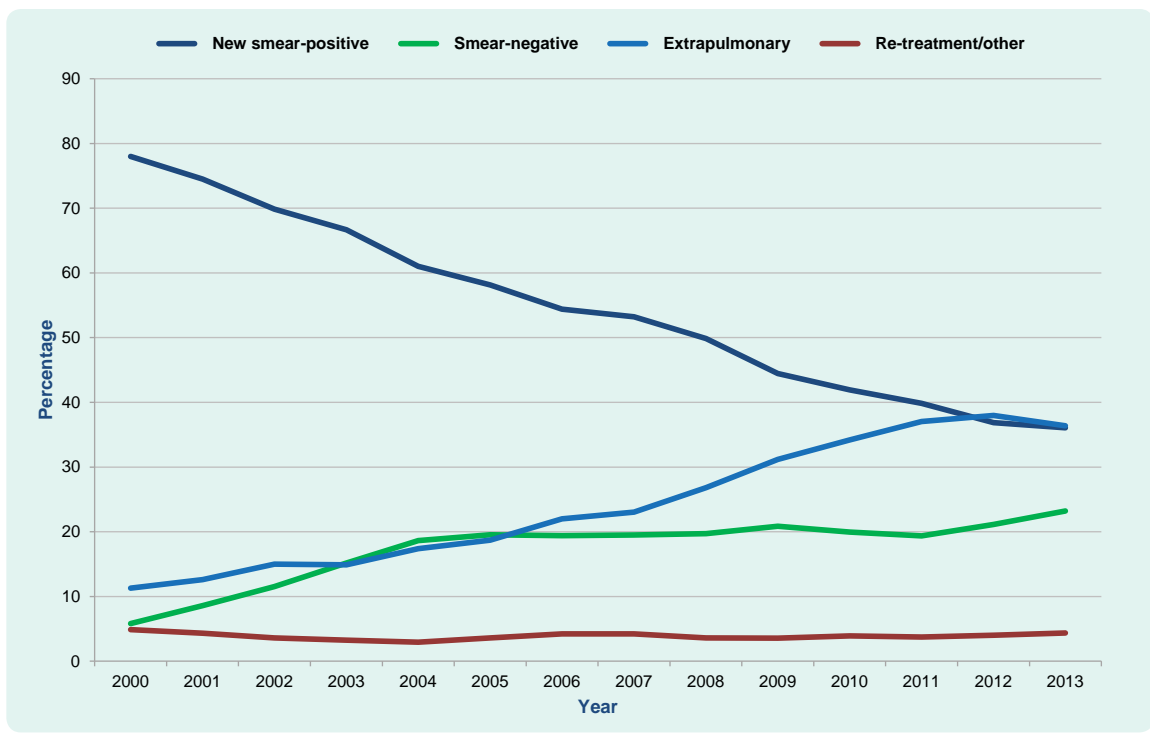

Figure 3c. Case notification rates for all forms of TB and smear-positive TB, Cambodia, 2000-2013

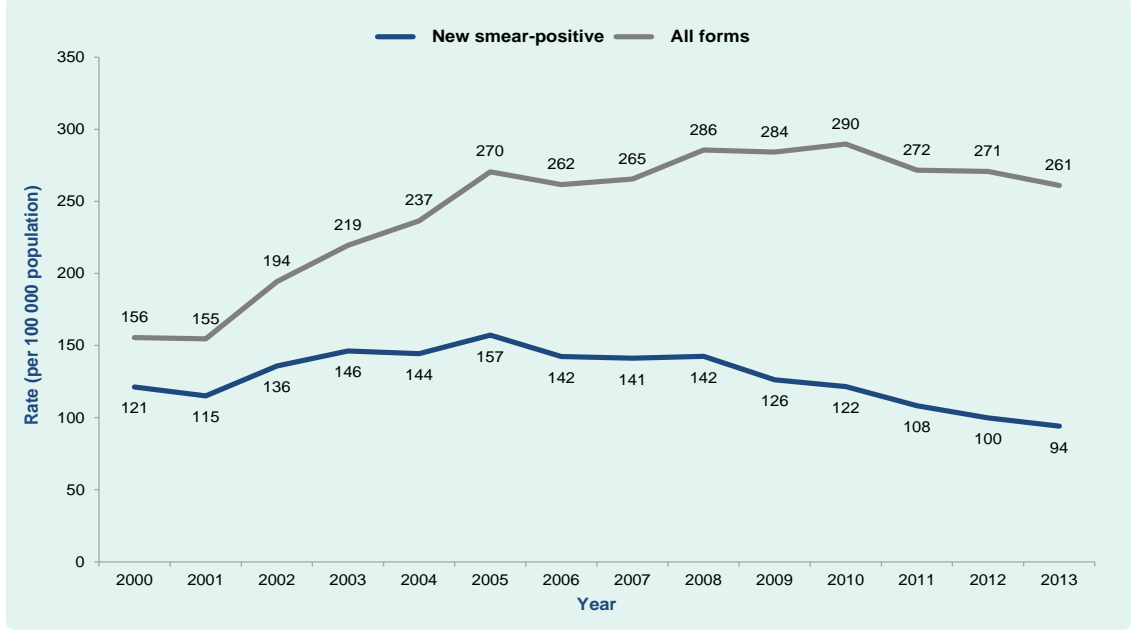


Figure 4. Number and case notification rate of new smear-positive TB cases by age and sex, Cambodia, 2011-2013

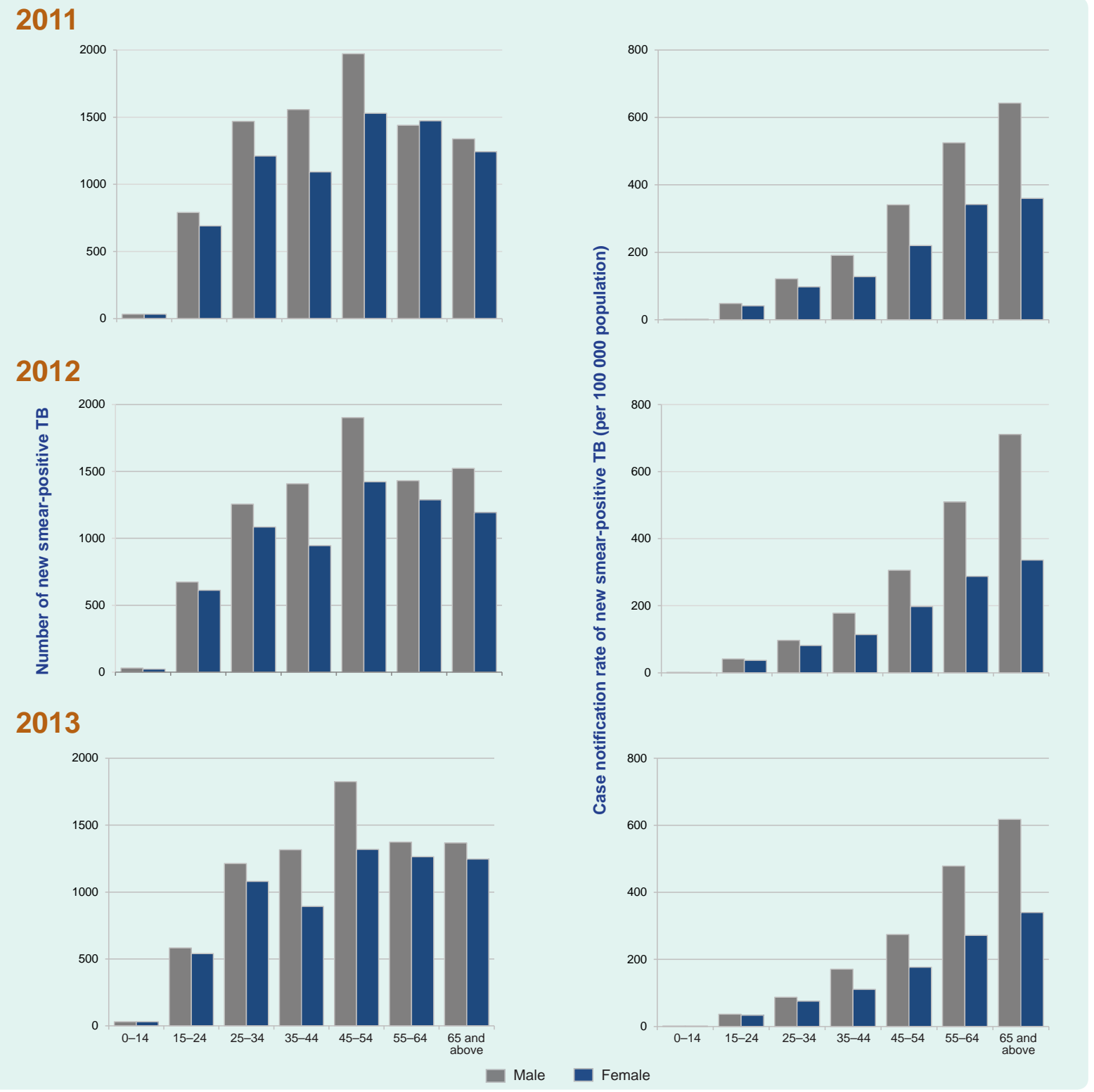

\section{TB notifications in the elderly}

In 2013, elderly people (aged 55 or over) accounted for $37 \%$ of new smear-positive TB notifications; CNRs of smear-positive TB in the elderly and nonelderly (people aged 54 or less) were 347 and 66 per 100000 population, respectively. By province, there was a positive correlation between elderly and nonelderly CNRs (Pearson's correlation coefficient 0.79, $P<0.001$; Figure 7). There were some provinces that had very different CNRs in these two groups, including Rattanakiri (109 per 100000 for the elderly and 37 per 100000 for the non-elderly) and Odor Meanchey (533 per 100000 for the elderly and 83 per 100000 for the non-elderly).

\section{TB notifications in children}

The NTP has expanded childhood TB services in recent years; in 2013, 13 of the 24 provinces routinely provide childhood TB services including case-finding, case management, contact tracing and provision of isoniazid preventive therapy. In 2013, the total number of TB notifications in children was $6916(17.7 \%$ of all notified TB cases), of which $87.0 \%$ and $12.1 \%$ were extrapulmonary and smear-negative, respectively. The proportion of TB in children among total notifications ranged from $1.3 \%$ to $39.4 \%$ across provinces (Table 2). Pailin City had the highest proportion (39.4\%) followed by Banteay Meanchey (36.3\%) and Battambang (33.5\%). 
Table 1. Number of notified TB cases by category and province, Cambodia, 2013

\begin{tabular}{|c|c|c|c|c|c|c|c|c|c|}
\hline Province & $\begin{array}{l}\text { New and } \\
\text { relapse }\end{array}$ & $\begin{array}{l}\text { Smear- } \\
\text { positive }\end{array}$ & $\begin{array}{c}\text { New cases } \\
\text { Smear- } \\
\text { negative }\end{array}$ & $\begin{array}{c}\text { Extra- } \\
\text { pulmonary }\end{array}$ & Relapse & $\begin{array}{l}\text { Re-treatment, } \\
\text { excluding } \\
\text { relapse }\end{array}$ & $\begin{array}{c}\text { Total } \\
\text { re-treatment }\end{array}$ & $\begin{array}{l}\text { Otherl } \\
\text { history } \\
\text { known }\end{array}$ & $\begin{array}{l}\% \text { of smear- } \\
\text { positive } \\
\text { among new } \\
\text { pulmonary }\end{array}$ \\
\hline Banteay Meanchey & 2038 & 584 & 460 & 970 & 24 & 3 & 27 & 110 & 55.9 \\
\hline Battambang & 2651 & 836 & 635 & 1147 & 33 & 9 & 42 & 74 & 56.8 \\
\hline Kampot & 1265 & 661 & 244 & 347 & 13 & 3 & 16 & 42 & 73.0 \\
\hline Kandal & 2635 & 1160 & 550 & 902 & 23 & 5 & 28 & 40 & 67.8 \\
\hline Kep & 78 & 37 & 11 & 30 & 0 & 0 & 0 & 0 & 77.1 \\
\hline Koh Kong & 181 & 92 & 30 & 58 & 1 & 0 & 1 & 0 & 75.4 \\
\hline Kompong Cham & 4233 & 1392 & 1021 & 1766 & 54 & 17 & 71 & 62 & 57.7 \\
\hline Kompong Chhnang & 1226 & 638 & 208 & 374 & 6 & 0 & 6 & 19 & 75.4 \\
\hline Kompong Som & 422 & 150 & 60 & 208 & 4 & 0 & 4 & 7 & 71.4 \\
\hline Kompong Speu & 2621 & 950 & 916 & 739 & 16 & 2 & 18 & 61 & 50.9 \\
\hline Kompong Thom & 1078 & 711 & 157 & 204 & 6 & 0 & 6 & 4 & 81.9 \\
\hline Kratie & 374 & 175 & 77 & 122 & 0 & 0 & 0 & 2 & 69.4 \\
\hline Mondolkiri & 47 & 36 & 4 & 5 & 2 & 0 & 2 & 0 & 90.0 \\
\hline National Hospital & 1310 & 369 & 377 & 488 & 76 & 13 & 89 & 121 & 49.5 \\
\hline Odor Meanchay & 583 & 270 & 78 & 230 & 5 & 0 & 5 & 11 & 77.6 \\
\hline Pailan City & 270 & 73 & 51 & 145 & 1 & 0 & 1 & 12 & 58.9 \\
\hline Phnom Penh & 2107 & 1143 & 383 & 548 & 33 & 9 & 42 & 22 & 74.9 \\
\hline Preah Vihear & 275 & 151 & 66 & 55 & 3 & 2 & 5 & 16 & 69.6 \\
\hline Prey Veng & 4345 & 997 & 863 & 2472 & 13 & 1 & 14 & 41 & 53.6 \\
\hline Pursat & 1075 & 439 & 305 & 321 & 10 & 0 & 10 & 19 & 59.0 \\
\hline Rattanakiri & 132 & 72 & 12 & 44 & 4 & 1 & 5 & 4 & 85.7 \\
\hline Siem Reap & 3067 & 1293 & 707 & 1044 & 23 & 9 & 32 & 137 & 64.7 \\
\hline Stung Treng & 251 & 136 & 19 & 95 & 1 & 0 & 1 & 0 & 87.7 \\
\hline Svay Rieng & 2109 & 556 & 762 & 766 & 25 & 0 & 25 & 284 & 42.2 \\
\hline Takeo & 3370 & 1161 & 1073 & 1123 & 13 & 0 & 13 & 150 & 52.0 \\
\hline Total & 37743 & 14082 & 9069 & 14203 & 389 & 74 & 463 & 1238 & 60.8 \\
\hline
\end{tabular}

CNRs of adults and children by province were weakly correlated (Pearson's correlation coefficient $0.34, P<0.107$; Figure 8), suggesting the scale of case-finding and registration activities for childhood TB relative to those for adult TB varies across provinces. Although provinces such as Siem Reap and Svay Rieng had high CNRs in adults, they had relatively low CNRs in children (Figure 8).

\section{DISCUSSION}

This analysis of routine TB surveillance data revealed several key findings that demonstrated improved programmatic indicators at the national level while highlighting some subnational gaps.

A decrease in new smear-positive cases provides some indication of the long-term impact of Cambodian's NTP. Case notifications can be driven by various determinants such as changes in case-finding efforts, laboratory quality and capacity, R\&R systems, case notification system as well as underlying TB incidence. ${ }^{10}$ While recognizing multiple contributors, the decrease of new smear-positive TB in Cambodia is likely due to the continued efforts of the NTP to detect and treat the most infectious cases through nationwide DOTS expansion. The National Prevalence Survey 2011 reported a $38 \%$ reduction of smear-positive TB prevalence from 2002 to $2011 .^{11}$ The decrease in the smear-positivity in spite of rise in population-screening rate could be another indication of the reduced prevalence of smearpositive cases.

The increase in smear-negative and extrapulmonary TB notifications could be explained by multiple epidemiological and operational factors including the expansion of childhood TB services, nationwide implementation of targeted active case-finding using mobile X-ray equipment and improved accessibility to $X$-ray and biopsy services through the decentralization of TB services. Increases in smear-negative notifications could also indicate a reduction in the most infectious 


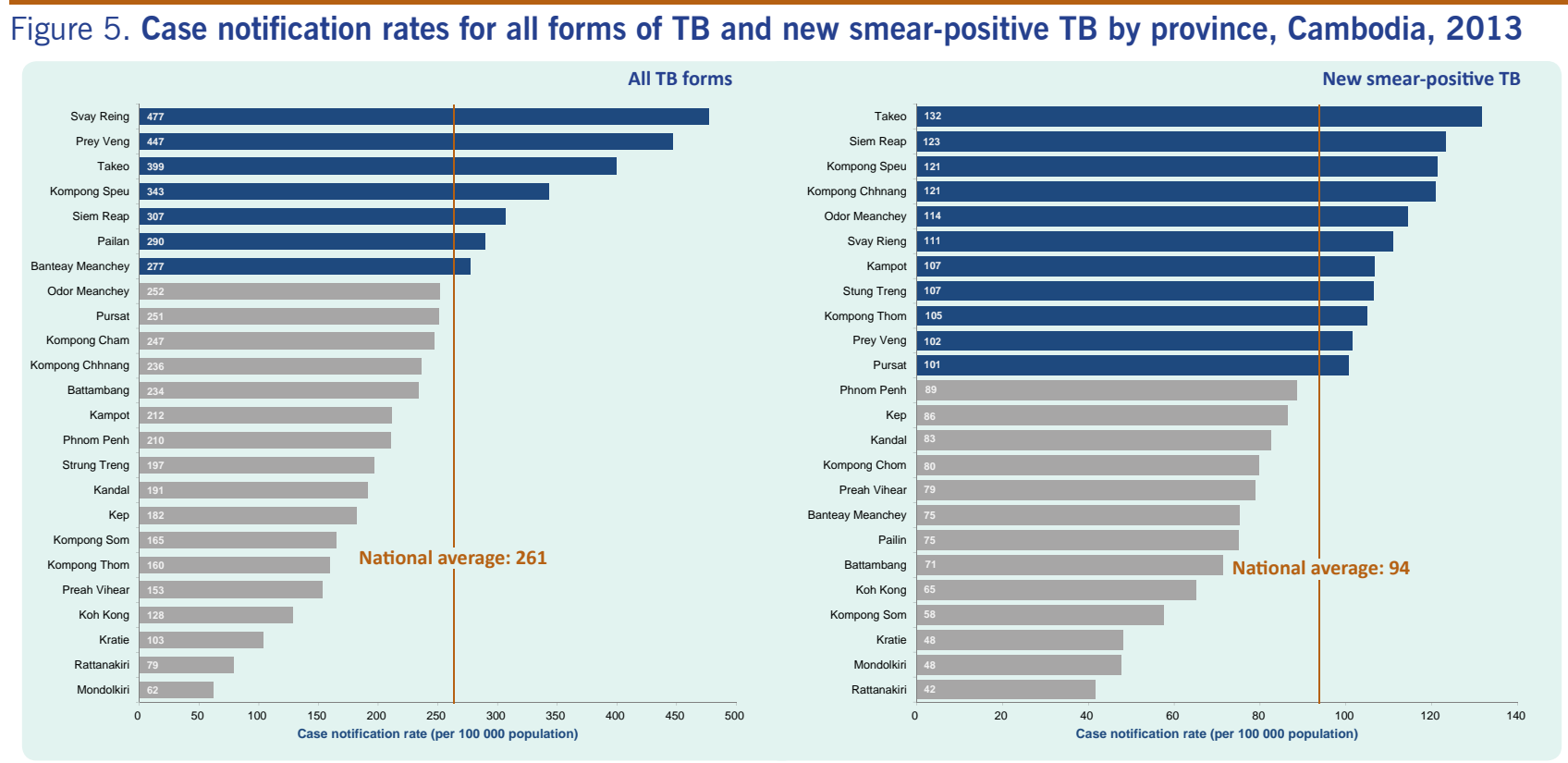

Note: The gray bars indicate those that are below national average while the blue bars indicate those that are above national average.

Figure 6. Case notification rates for all forms of TB and new smear-positive TB by operational district, Cambodia, 2013

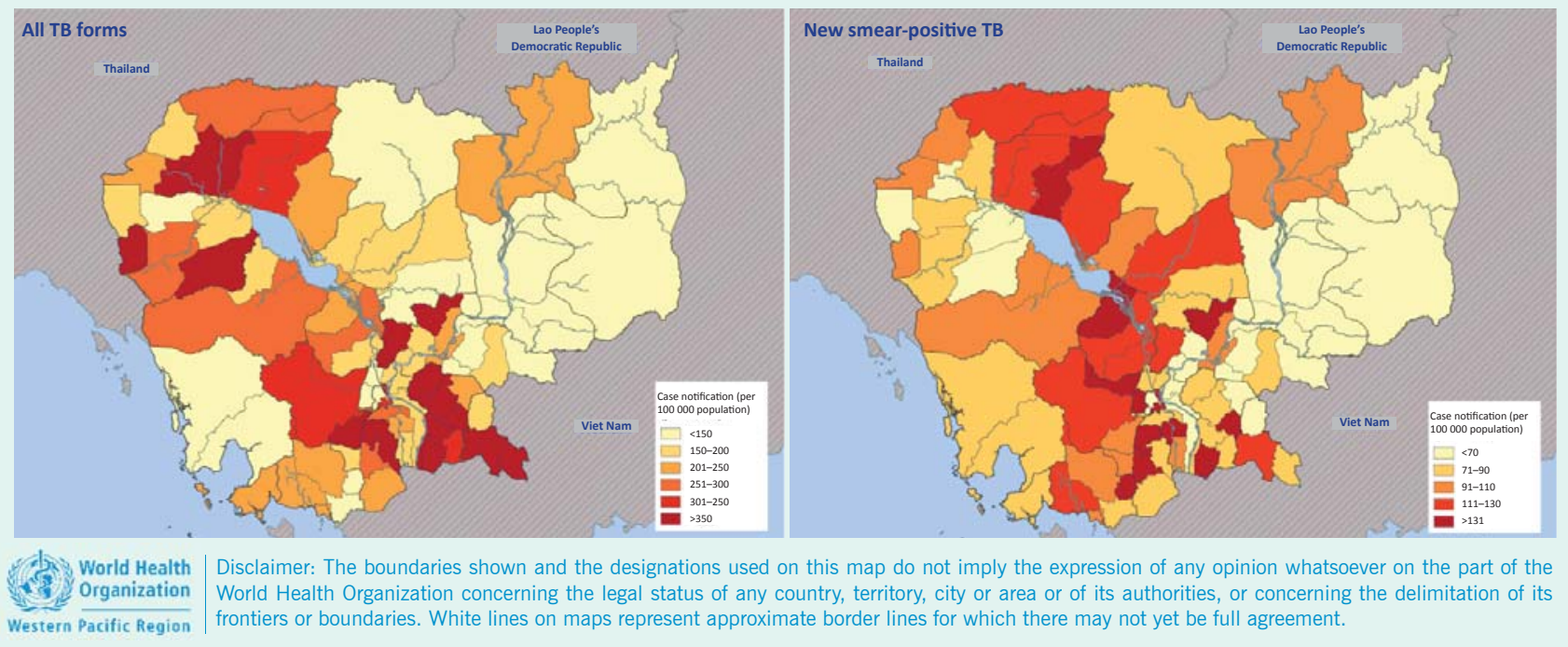

cases in the community. Despite the significant reduction in the prevalence of smear-positive TB, the 2011 National Prevalence Survey found a twofold higher prevalence of smear-negative, culture-positive (SNCP) cases. ${ }^{11}$ However, the notifications of smear-negative TB in 2013 was less than that of smear-positive TB (9069 vs 14 082), suggesting that many SNCP cases are still undiagnosed within the current routine diagnostic algorithm. To detect these undiagnosed SNCP cases, the existing diagnostic tool should be strengthened, for example, by promoting widespread use of $\mathrm{X}$-ray and Xpert MTB/RIF assay, ensuring cost-effectiveness.
Subnational analysis can help identify areas with possible undiagnosed cases. Wong et al. found a negative association between household poverty rates and smear-positive CNR in Cambodia and highlighted the potential to geo-target areas with high poverty rates for case-finding. ${ }^{7}$ Our subnational analysis, comparing population-screening rates and smear-positivity rates, provided additional criteria for geo-targeted case-finding. Provinces with a low population-screening rate yet a high smear-positivity rate can still strengthen case-finding to help reduce the smear-positivity rate. Prioritizing these areas may ensure more effective case-finding. 
Figure 7. Scatterplot of elderly and non-elderly case notification rates for new smear-positive TB by province, Cambodia, 2013

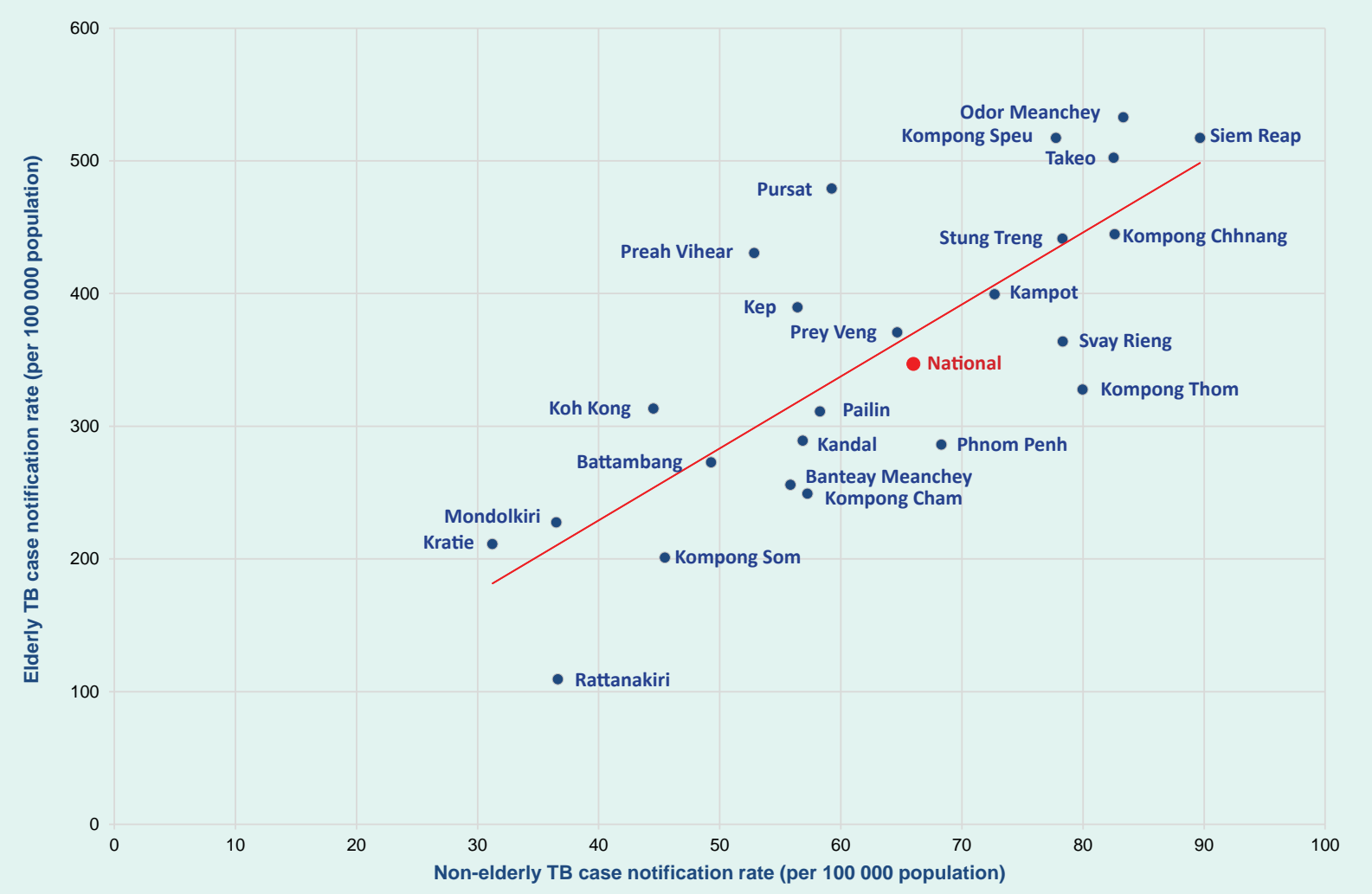

Note: Age-specific, province-specific population data were sourced from the projected population for 2013 in the 2008 Census and used for the CNR calculation.

Figure 8. Scatterplot of TB case notification rate in adults and children by province, Cambodia, 2013

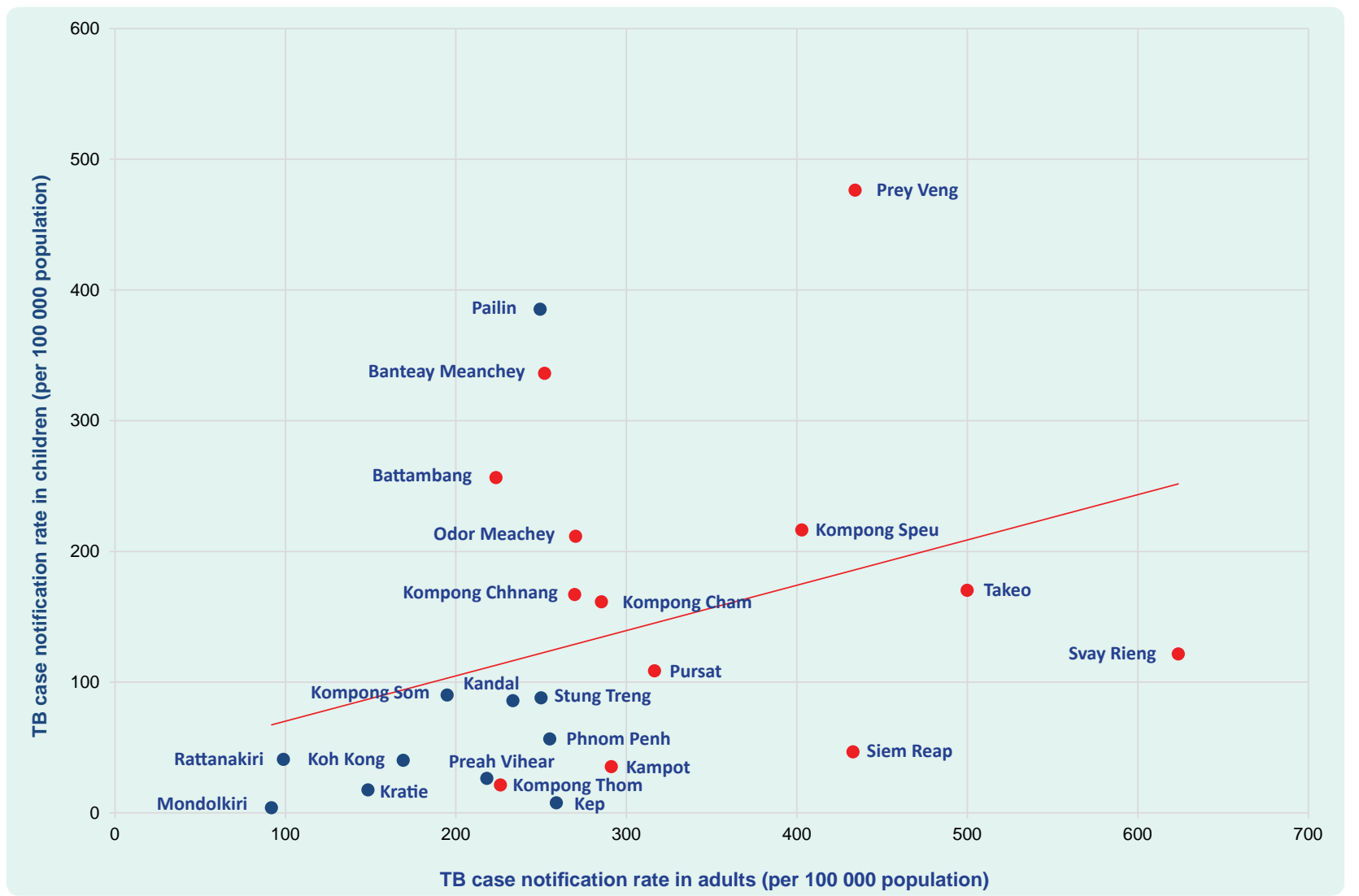

Red dots represent provinces with routine childhood TB services available in 2013, while blue dots represent provinces with routine childhood TB services unavailable in 2013. 
Table 2. Number of notified TB cases in children by province, Cambodia, 2013

\begin{tabular}{|c|c|c|c|c|c|c|c|c|c|}
\hline \multirow{2}{*}{ Province } & \multirow{2}{*}{$\begin{array}{l}\text { Total } \\
\text { cases }\end{array}$} & \multicolumn{2}{|c|}{ Extrapulmonary } & \multicolumn{2}{|c|}{ Smear-negative } & \multicolumn{2}{|c|}{ All other } & \multirow{2}{*}{$\begin{array}{l}\% \text { of childhood } \\
\text { TB among total } \\
\text { notifications }\end{array}$} & \multirow{2}{*}{$\begin{array}{l}\text { Childhood TB } \\
\text { service routinely } \\
\text { available in } 2013\end{array}$} \\
\hline & & $n$ & $\%$ & $n$ & $\%$ & $\mathbf{n}$ & $\%$ & & \\
\hline Banteay Meanchey & 780 & 682 & 87.4 & 98 & 12.6 & 0 & 0.0 & 36.3 & + \\
\hline Battambang & 915 & 697 & 76.2 & 216 & 23.6 & 2 & 0.2 & 33.5 & + \\
\hline Kampot & 68 & 64 & 94.1 & 3 & 4.4 & 1 & 1.5 & 5.2 & + \\
\hline Kandal & 346 & 324 & 93.6 & 22 & 6.4 & 0 & 0.0 & 12.9 & \\
\hline Kep & 1 & 1 & 100 & 0 & 0.0 & 0 & 0.0 & 1.3 & \\
\hline Koh Kong & 18 & 17 & 94.4 & 1 & 5.6 & 0 & 0.0 & 9.9 & \\
\hline Kompong Cham & 870 & 801 & 92.1 & 59 & 6.8 & 10 & 1.1 & 20.2 & + \\
\hline Kompong Chhnang & 291 & 199 & 68.4 & 85 & 29.2 & 7 & 2.4 & 23.4 & + \\
\hline Kompong Som & 67 & 66 & 98.5 & 0 & 0.0 & 1 & 1.5 & 15.6 & + \\
\hline Kompong Speu & 548 & 361 & 65.9 & 176 & 32.1 & 11 & 2.0 & 20.4 & + \\
\hline Kompong Thom & 47 & 29 & 61.7 & 13 & 27.7 & 5 & 10.6 & 4.3 & + \\
\hline Kratie & 22 & 21 & 95.5 & 1 & 4.5 & 0 & 0.0 & 5.9 & \\
\hline Mondolkiri & 1 & 0 & 0.0 & 1 & 100 & 0 & 0.0 & 2.1 & \\
\hline National Hospital & 138 & 99 & 71.7 & 36 & 26.1 & 3 & 2.2 & 9.6 & \\
\hline Odor Meanchay & 157 & 150 & 96 & 6 & 3.8 & 1 & 0.6 & 26.4 & + \\
\hline Pailan City & 111 & 105 & 94.6 & 5 & 4.5 & 1 & 0.9 & 39.4 & \\
\hline Phnom Penh & 80 & 69 & 86 & 3 & 3.8 & 8 & 10.0 & 3.7 & \\
\hline Preah Vihear & 17 & 16 & 94.1 & 0 & 0.0 & 1 & 5.9 & 5.8 & + \\
\hline Prey Veng & 1433 & 1392 & 97.1 & 37 & 2.6 & 4 & 0.3 & 32.7 & \\
\hline Pursat & 150 & 118 & 78.7 & 31 & 20.7 & 1 & 0.7 & 13.7 & + \\
\hline Rattanakiri & 24 & 22 & 91.7 & 2 & 8.3 & 0 & 0.0 & 17.5 & \\
\hline Siem Reap & 160 & 154 & 96.3 & 4 & 2.5 & 2 & 1.3 & 5.0 & + \\
\hline Stung Treng & 37 & 37 & 100 & 0 & 0.0 & 0 & 0.0 & 14.7 & \\
\hline Svay Rieng & 178 & 150 & 84.3 & 26 & 14.6 & 2 & 11 & 7.4 & + \\
\hline Takeo & 457 & 444 & 97.2 & 9 & 2 & 4 & 0.9 & 13.0 & + \\
\hline Total & 6916 & 6018 & 87.0 & 834 & 12.1 & 64 & 0.9 & 17.7 & \\
\hline
\end{tabular}

Elderly TB cases accounted for $37 \%$ of the total notifications in 2013. This was lower than the 55\% of smear-positive cases reported for the elderly during the 2011 National Prevalence Survey, ${ }^{11}$ suggesting that many elderly cases may still be undiagnosed. We reported on provinces with lower elderly CNRs relative to non-elderly CNRs that may have many undiagnosed elderly TB cases. These provinces may therefore benefit from interventions that systematically screen the elderly for TB.

Internationally, the proportion of TB in children among total notifications varies from $3 \%$ to more than $25 \% .^{12}$ Through rapidly expanding childhood TB services in Cambodia's NTP, the proportion of TB notifications in children in 2013 reached $17.7 \%$ of all notifications; however, subnational differences exist. Differences at the subnational level in the proportion of childhood TB, as well as CNRs in children relative to
CNRs in adults, may help identify areas with possible over-diagnosis or underreporting. For instance, provinces with high CNR in adults but low CNR in children may have many undiagnosed or unreported childhood TB cases. In Cambodia, where the role of the private sector in public health programmes has been rapidly expanding, engagement of the private sector in national TB surveillance system needs strengthening. Given the complexity in diagnosing childhood TB that requires thorough assessment of all evidence, ${ }^{13}$ possible overdiagnosis of TB in children should be investigated in provinces with high CNRs in children but relatively low CNRs in adults.

There are some inherent limitations in using surveillance data. Although the NTP has a strengthened R\&R system, including training and supervision, the reliability of the data is heavily dependent on local facility and staff capacity. Operational challenges in 
nationwide data collection using paper-based forms and manual data entry might have led to inconsistencies in some of the data reported. While Public-Private Mix initiatives to control TB have recently expanded, TB patients diagnosed in the private sector may not be notified to the NTP, limiting the capture of all TB cases. TB notification may not be a direct measure or close proxy of TB incidence, particularly when the level of case detection is insufficient. ${ }^{3,7}$

In many developing countries, TB surveillance data is often underutilized despite substantial resources invested in the data collection and its availability. For effective use of surveillance data, subnational analysis should be strengthened as it can provide robust evidence to set criteria for geo-targeted case-finding. If combined with a demographic-targeted approach, the subnational analysis enables the progamme to target a more specific segment of the population, which further encourages strategic resource allocation. However, strengthening data quality and management is of particular importance at every level to ensure reliability and validity of the analysis. Increasing efforts should be encouraged to create and maintain a well designed database enabling systematic data entry and analysis. Similarly, existing data verification tools and methods to ensure internal/ external consistency of surveillance data should be actively employed. ${ }^{14}$ Development of innovative tools to reproduce graphics and tables could further facilitate the periodic data analysis and dissemination at different operational levels, which helps motivate programme staff in their routine activities. Finally, the ultimate goal of such analyses is to explore reasons behind the findings and inform corrective actions, always involving diverse voices from the field.

\section{Conflicts of interest}

None declared.

\section{Funding}

None.

\section{Acknowledgements}

The authors wish to thank all health-care workers at the front lines of service delivery of TB diagnosis and treatment in Cambodia. The authors extend our thanks to all staff members of the national and subnational tuberculosis programmes of Cambodia for their tremendous efforts in data collection and reporting.

\section{References:}

1. The Stop TB Strategy. Building on and enhancing DOTS to meet the TB-related Millennium Development Goals. Geneva, World Health Organization, 2006 (http://www.who.int/tb/publications/2006/ stop_tb_strategy.pdf, accessed 28 January 2015).

2. Nishikiori N, Morishita F. Using tuberculosis surveillance data for informed programmatic decision-making. Western Pacific Surveillance and Response Journal, 2013, 4(1):1-3. doi:10.5365/wpsar.2013.4.1.007 pmid:23908948

3. Castro KG. Tuberculosis surveillance: data for decisionmaking. Clinical Infectious Diseases, 2007, 44:1268-1270. doi:10.1086/514351 pmid:17443461

4. Revised TB recording and reporting forms and registers - version 2006. Geneva, World Health Organization, 2006 (http://www.who.int/tb/err/rr final forms en.pdf, accessed 28 January 2015)

5. Tuberculosis Report 2013 - Kingdom of Cambodia. Phnom Penh, National Center for Tuberculosis and Leprosy Control, Ministry of Health, 2014.

6. Hill PS, Tan Eang M. Resistance and renewal: health sector reform and Cambodia's national tuberculosis programme. Bulletin of the World Health Organization, 2007, 85:631-636. doi:10.2471/ BLT.06.036822 pmid:17768522

7. Wong MK et al. The association between household poverty rates and tuberculosis case notification rates in Cambodia, 2010 Western Pacific Surveillance and Response Journal, 2013, 4:2533. doi:10.5365/wpsar.2013.4.1.002 pmid:23908952

8. Revised definitions and reporting framework for tuberculosis Geneva, World Health Organization, 2013 (http://apps.who. int/iris/bitstream/10665/79199/1/9789241505345 eng.pdf accessed 28 January 2015).

9. Frieden $\mathrm{T}$, editor. Toman's tuberculosis case detection, treatment and monitoring: questions and answers, Second edition. Geneva, World Health Organization, 2004 (http:// whqlibdoc.who.int/publications/2004/9241546034.pdf accessed 28 January 2015)

10. Tuberculosis in the region of the Americas - Regional Report 2011 Epidemiology, Control and Financing. Washington, DC, Pan American Health Organization, Regional Office for the Americas of the World Health Organization, 2012 (http://www. paho.org/hq/index.php?option =com_docman\&task $=$ doc view\&gid $=22953 \&$ Itemid $=$, accessed 28 January 2015)

11. Report: Second National Tuberculosis Prevalence Survey Cambodia, 2011. Phnom Penh, National Center for Tuberculosis and Leprosy Control, Ministry of Health, 2012.

12. Guidance for national tuberculosis programmes on the management of tuberculosis in children. Geneva, World Health Organization, 2006 (http://whqlibdoc.who.int/hq/2006/WHO HTM_TB_2006.371_eng.pdf, accessed 28 January 2015).

13. Guidance for national tuberculosis programmes on the management of tuberculosis in children, Second edition. Geneva, World Health Organization, 2014 (http://apps.who.int/ medicinedocs/documents/s21535en/s21535en.pdf, accessed 28 January 2015).

14. Standards and benchmarks for tuberculosis surveillance and vital registration systems: checklist and user guide. Geneva, World Health Organization, 2014 (http://apps.who.int/iris/ bitstream/10665/112673/1/9789241506724 eng.pdf accessed 28 January 2015). 


\title{
Mass poisoning after consumption of a hawksbill turtle, Federated States of Micronesia, 2010
}

\author{
Boris I Pavlin, a Jennie Musto, ${ }^{b}$ Moses Pretrick, ${ }^{c}$ Joannes Sarofalpiy, ${ }^{c}$ Perpetua Sappa, ${ }^{d}$ Siana Shapucy ${ }^{d}$ and \\ Jacobus Kool ${ }^{b}$ \\ Correspondence to Boris I Pavlin (e-mail: pavlinb@wpro.who.int).
}

Background: Marine turtles of all species are capable of being toxic. On 17 October 2010, health authorities in the Federated States of Micronesia were notified of the sudden death of three children and the sickening of approximately 20 other people on Murilo Atoll in Chuuk State. The illnesses were suspected to be the result of mass consumption of a hawksbill turtle (Eretmochelys imbricata). An investigation team was assembled to confirm the cause of the outbreak, describe the epidemiology of cases and provide recommendations for control.

Methods: We conducted chart reviews, interviewed key informants, collected samples for laboratory analysis, performed environmental investigations and conducted a cohort study.

Results: Four children and two adults died in the outbreak and 95 others were sickened; $84 \%$ of those who ate the turtle became ill $(n=101)$. The relative risk for developing illness after consuming the turtle was 11.1 (95\% confidence inteval: 4.8-25.9); there was a dose-dependent relationship between amount of turtle meat consumed and risk of illness. Environmental and epidemiological investigations revealed no alternative explanation for the mass illness. Laboratory testing failed to identify a causative agent.

Conclusion: We concluded that turtle poisoning (also called chelonitoxism) was the cause of the outbreak on Murilo. The range of illness described in this investigation is consistent with previously reported cases of chelonitoxism. This devastating incident highlights the dangers, particularly to children, of consuming turtle meat. Future incidents are certain to occur unless action is taken to alter turtle-eating behaviour in coastal communities throughout the world.

$\mathrm{M}$ arine turtles of all species, particularly hawksbill turtles (Figure 1 ), can contain a variety of toxins including heavy metals (e.g. cadmium and mercury), organic compounds (e.g. pesticides such as chlordane and polychlorinated biphenyls) and biotoxins such as those produced by various blue-green algae on which turtles feed (e.g. lyngbyatoxin $A$ from Lyngbya majuscula). ${ }^{1}$ Intoxication from consumption of marine turtles is called chelonitoxism. Symptoms of chelonitoxism vary according to the toxin and include gastrointestinal symptoms of nausea, vomiting and oro-esophageal ulceration, sometimes followed in severe cases by neurologic signs such as paresis, agitation and coma. ${ }^{1,2}$ Despite these hazards and despite the endangered status of all marine turtle species, these turtles are eaten by coastal communities throughout the world. $^{1}$

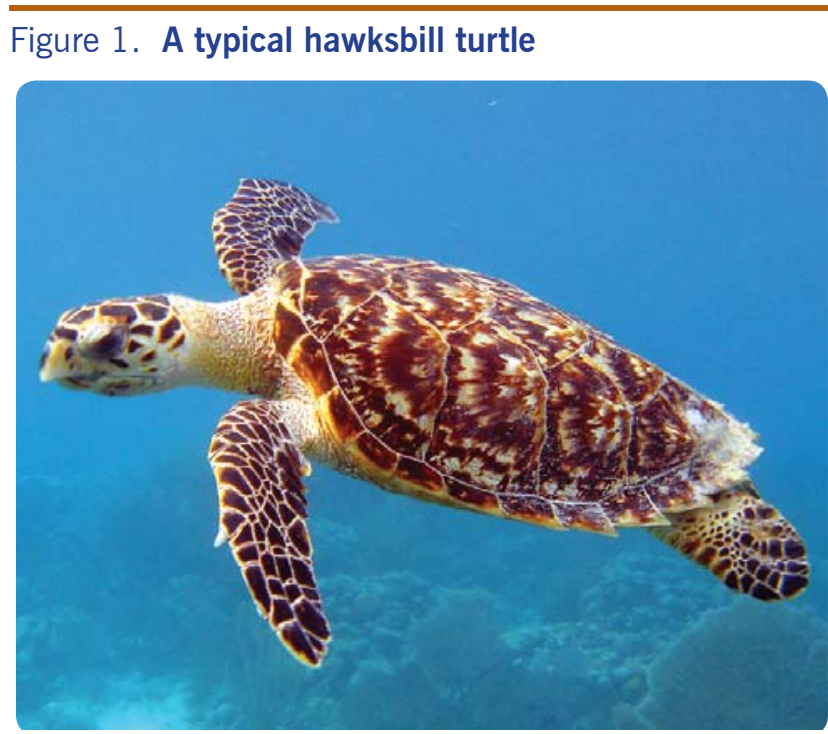

Source: http://commons.wikimedia.org/wiki/File:Hawksbill_Sea_Turtle_ Carey de Concha_(5840602412).jpg.

\footnotetext{
Office of the WHO Representative in Papua New Guinea, Port Moresby, Papua New Guinea.

Division of Pacific Technical Support, World Health Organization, Suva, Fiji.

Department of Health and Social Affairs, Government of the Federated States of Micronesia, Pohnpei, Federated States of Micronesia.

Chuuk State Hospital, Chuuk State Department of Health Services, Chuuk, Federated States of Micronesia.

Submitted: 9 September 2014; Published: 26 January 2015

doi: 10.5365/wpsar.2014.5.3.006
} 
The island nation of the Federated States of Micronesia comprises of 607 islands in the North Pacific Ocean (Figure 2). On Sunday, 17 October 2010, the Federated States of Micronesia Department of Health and Social Affairs and the World Health Organization (WHO) were notified of the sudden death of three children and the sickening of approximately 20 other people on Murilo Atoll, a small, isolated island with a population of approximately 330, located $161 \mathrm{kms}$ from the main population centre of Weno Island in Chuuk State. The illnesses were suspected to be the result of mass consumption of a hawksbill turtle (Eretmochelys imbricata) that had been prepared and served on the afternoon of 15 October. Shortly after the initial report, the deaths of another child and two hospitalized adults were reported; dozens of residents developed sore throats, and several dogs died suddenly.

A response team set up a field hospital on Murilo, and a investigation team was sent to confirm the cause of the outbreak, describe the epidemiology of cases and provide recommendations to prevent additional cases or outbreaks.

\section{METHODS}

\section{Hypothesis generation}

The outbreak investigation team held a community meeting on Murilo on 25 October to explain the purpose of the investigation, gather initial information and answer questions from community members. On the morning of 26 October, the team interviewed all 20 community members who did not eat the turtle but who reported feeling ill to determine whether there was any other threat besides turtle poisoning that could explain the reported illnesses.

Information on all known ill persons was reviewed to determine onset dates and whether there was an ongoing environmental threat or an infectious condition. Key informants from the community were interviewed about foods consumed as part of the turtle feast; presence of unusual environmental conditions such as chemical spills, algal blooms, fish die-offs and changes in reef ecology; and possibility of infectious etiology.

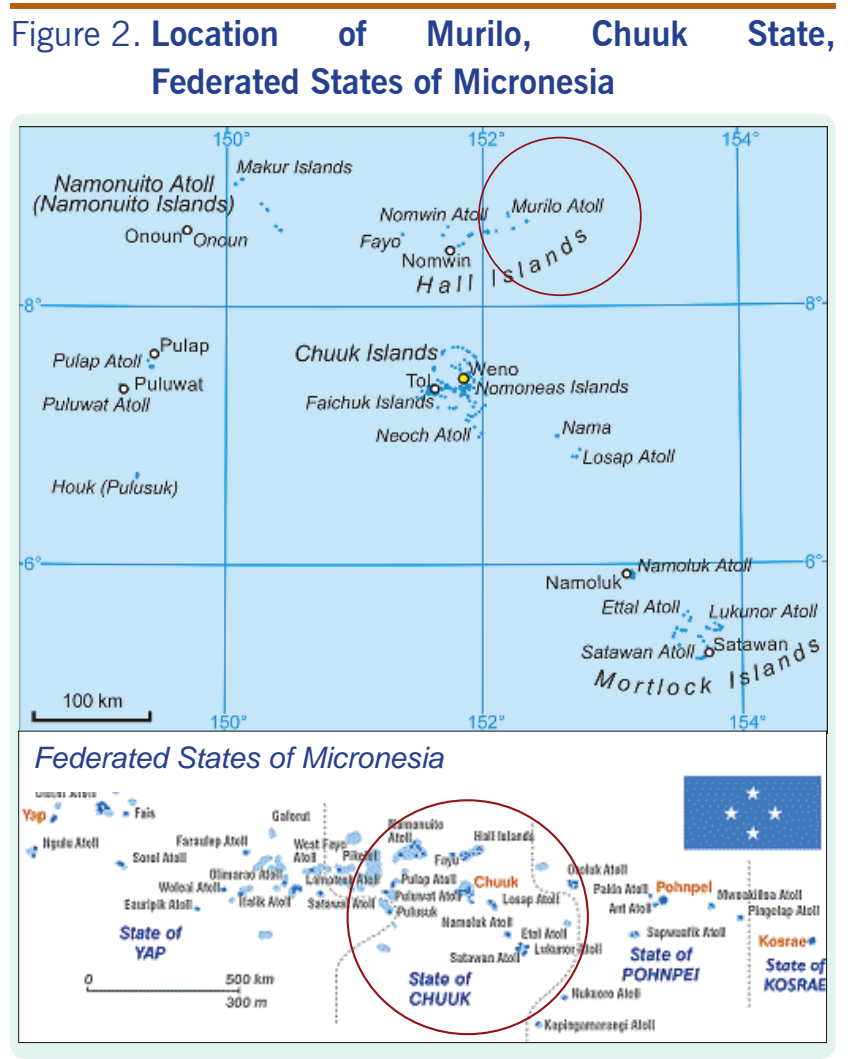

Source: Chuuk State map was reproduced from Wikimedia Commons, the free media repository (http://commons.wikimedia.org/wiki/File:Chuuk.png), and the inset map of the Federated States of Micronesia was reproduced from WHO Division of Pacific Technical Support (http://www.wpro.who. int/southpacific/pacelf/countries/fsm/en/).

\section{Case series}

A case was defined as a resident of Murilo who had sudden death, sore throat, mouth sores or excessive thirst with onset of illness between 15 and 28 October. All cases who had eaten turtle (or were breastfed by mothers who had eaten turtle) were interviewed to determine the amount of turtle consumed, onset of illness and clinical features. Proxy interviews were conducted with the local health assistant and family members of the six deceased cases; the medical charts of the two decedents who had been hospitalised were also reviewed.

\section{Cohort study}

A retrospective cohort study was conducted to determine risk factors for illness using the same case definition as per the case series. All persons present at the turtle feast and their household members were 
Figure 3. Cases of turtle poisoning by date of onset of illness

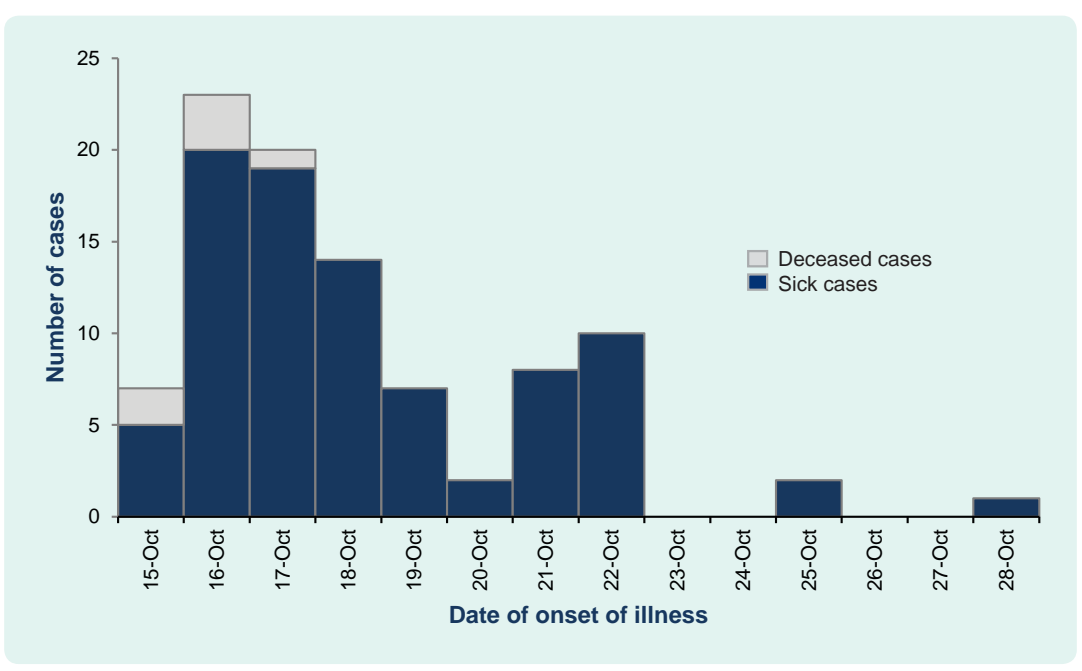

interviewed (or proxy interviewed if deceased) with a standardized questionnaire. This comprised questions on demographics, preparation and consumption of turtle, consumption of other foods and drinks and illness. The quantity of turtle soup consumed was reported as multiples of a $70 \mathrm{~mL}$ jar. Data were analysed using Microsoft Excel 2003, Epilnfo 3.5.1 and STATA 9. Univariate logistic regression was used to examine the relationship between illness and the risk factors of age, sex, village, involvement in turtle preparation and consumption of turtle (no other food items were common to more than a few people). Any risk factors approaching significance $(P<0.2)$ in univariate analysis were retained for multivariate logistic regression. As this research was part of an emergency public health response, no institutional review board consultation was needed.

\section{Environmental investigation}

Members of the investigation team together with an authority from the Chuuk Department of Marine Resources conducted an environmental assessment to look for evidence of contamination. Additionally, every effort was made to secure food samples from the turtle feast for laboratory testing.

\section{Laboratory testing}

Samples of patient sera, turtle scutes (shell plates) and bones, internal organs from a deceased dog and algae were sent to Forensic and Scientific Services, Queensland, Australia. Testing was conducted for the following marine toxins: lyngbyatoxin $A$, debromoaplysiatoxin, okadaic acid, gymnodimine, pectenotoxin 2 and dinophysistoxins.

Ethics approval was not required as this investigation was conducted as part of a public health intervention. Informed consents were obtained from all participants and for deceased cases from their next-of-kin.

\section{RESULTS}

\section{Hypothesis generation}

Of the 20 ill people who did not eat turtle, 10 were diagnosed as influenza-like illness. The remaining 10 were attributed to various other ailments. All cases had a date of illness onset on or before 22 October, with the exception of three outliers (Figure 3 ). Many residents of Murilo reported eating reef fish and other local foods around the time of the turtle feast and they did not become ill.

The turtle was captured alive from the Murilo lagoon using a hook and line (no chemicals) approximately one week before the turtle feast and was apparently healthy. It was kept alive on land, but it died in the early part of the day of the feast, thought to be from heat exhaustion. The turtle was prepared simply by chopping all meat and organs into a single pot for cooking; it was not gutted. The turtle was then boiled in fresh water for two to three hours, after which the contents of the first pot were further chopped and boiled a second time after discarding and replacing the water. Each household 
(including members of the extended family) was given a bowl of the turtle stew. In almost all instances, the stew was consumed immediately after it was served. Each household ate its own variety of accompaniments with the stew (e.g. rice, breadfruit, taro); there were no other common foods served at the feast.

The two adult brothers who died were involved in the butchering and cooking of the turtle. They were reported to have eaten a large amount of turtle at the feast (but not more than several others); it was also reported that the brothers may have eaten large amounts of turtle during cooking and preparation.

Residents reported that sea turtles were consumed on the island approximately twice a month, with most being green sea turtles (Chelonia mydas). There had never been an incident related to turtle poisoning on Murilo before this outbreak. Other hawksbill turtles had been consumed in the past year without incident. Residents were not aware of any hazard associated with consuming sea turtles.

\section{Case series}

Of the 120 people who consumed turtle (or who were breastfed by mothers who ate turtle), 101 ill people met the case definition. Females accounted for $51 \%$ of cases $(n=52)$ and the median age was 17 years (range $=$ 5 weeks to 70 years). The median incubation period from turtle consumption to illness was two days (range $=$ 2 hours to 13 days) (Figure 3 ). Medical attention was sought by $37 \%$ of cases $(n=37)$. Interviews were conducted 12 days after the exposure; only 27 of the 101 ill people had recovered at the time of interview. All surviving patients have reportedly since recovered.

Sore throat was the most frequently reported symptom, occurring in $84 \%$ of cases for whom responses were available $(n=76)$. Other commonly reported symptoms included mouth pain $(78 \%, n=72)$, thirst $(71 \%, n=66)$ and burning throat $(54 \%, n=47$; Table 1).

Eight cases were breastfed infants whose mothers had consumed turtle, although two infants also consumed turtle themselves.

Several patients whose mouths were examined had pale yellow exudative lesions of the oropharynx and
Table 1. Number and proportion of reported symptoms in cases, Murilo, 2010*

\begin{tabular}{lccc}
\hline \multicolumn{1}{c}{ Symptom } & Number & Respondents & Percentage (\%) \\
\hline Sore throat & 76 & 90 & 84 \\
Mouth pain & 72 & 92 & 78 \\
Thirst & 66 & 93 & 71 \\
Burning throat & 47 & 87 & 54 \\
Fever & 40 & 94 & 43 \\
Cough & 35 & 93 & 38 \\
Vomiting & 29 & 94 & 31 \\
Abdominal pain & 26 & 93 & 28 \\
Epigastric pain & 22 & 87 & 25 \\
Constipation & 20 & 94 & 21 \\
\hline
\end{tabular}

* Number of respondents varies as not all patients responded to all questions because several of the patients were infants.

tongue. This was ubiquitous among those who had eaten turtle and had presented to the field hospital.

\section{Fatal cases}

Case 1 was a previously healthy five-year-old girl who consumed turtle meat at approximately $16: 00$ on 15 October and then vomited once approximately 24 hours after the meal. Her only other reported symptom was thirst; however, she refused all oral liquids when offered. Within 36 hours after consumption, on the morning of 17 October, she developed seizures and died shortly afterwards.

Case 2 was a previously healthy two-year-old girl and the sister of Case 1 who died within minutes of her sibling. The mother reported that the child woke irritable, distressed and with abdominal pain at approximately 03:00 on 16 October, was settled after breastfeeding but died shortly after. Case 2 consumed a small amount of turtle meat but was breastfed by her mother within three hours after she had eaten turtle.

Case 3 was a previously healthy two-year-old boy who did not consume turtle but was breastfed by his mother after she consumed large amounts of turtle. Case 3 had two episodes of diarrhoea on the night of 16 October and another in the early morning of 17 October. He died shortly thereafter.

Case 4 was a previously healthy 21-year-old man who consumed turtle at approximately $16: 00$ on 15 October. Approximately two hours later he experienced 
Table 2. Dose-response of odds of illness for amounts of turtle consumed, Murilo, 2010

\begin{tabular}{cccc}
\hline \multirow{2}{*}{ Dose* $^{*}$ Odds ratio } & \multicolumn{2}{c}{$95 \%$ confidence interval } \\
& & Lower & Upper \\
\hline None & 1.00 (Reference) & \\
$<1$ & 32.0 & 9.4 & 108.7 \\
1 & 61.0 & 10.4 & 358.4 \\
2 & 122.0 & 12.9 & 1156.0 \\
3 & 146.4 & 15.6 & 1367.5 \\
$4+$ & 77.3 & 22.0 & 270.8 \\
Chi-square $=111.75$ & & & \\
$p$-value $<0.0001$ & & & \\
\hline
\end{tabular}

* 1 dose was a $70 \mathrm{~mL}$ cup. This table does not include five cases who were exclusively breastfed and 11 cases where the amount consumed is unknown.

nausea but did not vomit. The following evening, he vomited more than 10 times and then eventually went to sleep. The next morning he felt better and went fishing. While at sea he developed haematemesis and was evacuated to Chuuk State Hospital on Weno Island by a nearby supply ship. On the boat he became restless and disoriented and did not recognize his relatives. Ambulance drivers on Weno noted the case had an unusual smell unlike anything they had experienced. At the hospital he was agitated and combative and required sedation. He was placed in the intensive care unit and administered intravenous fluids: mannitol for presumed ciguatera intoxication and dexamethasone for presumed cerebral oedema. A neurologic exam found the patient was profoundly lethargic with pinpoint pupils and hyperreflexia in all extremities. Vital signs were within normal ranges except for an elevated respiratory rate. Laboratory values were unremarkable except for mild neutrophilia. Radiography was unremarkable.

Over the following two days, the patient remained profoundly lethargic and developed sustained clonus and an upward plantar reflex. His chemistries were notable for markedly elevated aminotransferases, amylase and creatinine. The patient remained in this state until 21 October when he developed respiratory distress requiring intubation and mechanical ventilation. He was removed from the ventilator at the request of his family in the early morning of 22 October and died at 04:00.
Case 5 was the brother of Case 4, a previously healthy 22-year-old man who consumed turtle at approximately 16:00 on 15 October. Three hours later he felt nauseated, did not vomit but reported thirst. On the following evening he vomited several times. On the third day he was noted to have headache, weakness, fever, disorientation and agitation. $\mathrm{He}$ was taken to the hospital intensive care unit on the morning of 19 October and placed on intravenous fluids: mannitol for presumed ciguatera intoxication and dexamethasone for presumed cerebral oedema. He was restless and disoriented but otherwise unremarkable. Initial radiography and laboratory investigation were also unremarkable.

Over the course of the evening the patient became agitated and combative and required sedation. On the evening of 21 October the patient developed severe respiratory distress, and he died at 01:00 on 22 October.

Case 6 was a previously healthy four-year-old boy who ate turtle on the evening of 15 October. He vomited on the evenings of 16 and 17 October, then appeared well until he reported a sore throat on 21 October. In the early morning of 22 October he vomited once, collapsed and died.

For all decedent cases, except for Case 4 whose serum was sent for laboratory analysis, no samples were taken and autopsies were not performed.

\section{Cohort study}

The cohort study included 186 residents of Murilo present at the turtle feast and their household members. Consumption of turtle stew had an attack rate of $84 \%$ (101/120). The relative risk of developing illness after consuming turtle stew in univariate analysis was 11.1 (95\% confidence interval: 4.8-25.9). In multivariate analysis, consumption of turtle was the only risk factor associated with illness; village and involvement in the preparation of turtle were not (data not shown).

There was a statistically significant dose-response relationship between the amount of turtle consumed and the odds of developing illness (Table 2) with the 
Figure 4. Abundant algae presumed to be Lyngbya majuscula, also known as "Mermaid's Hair"

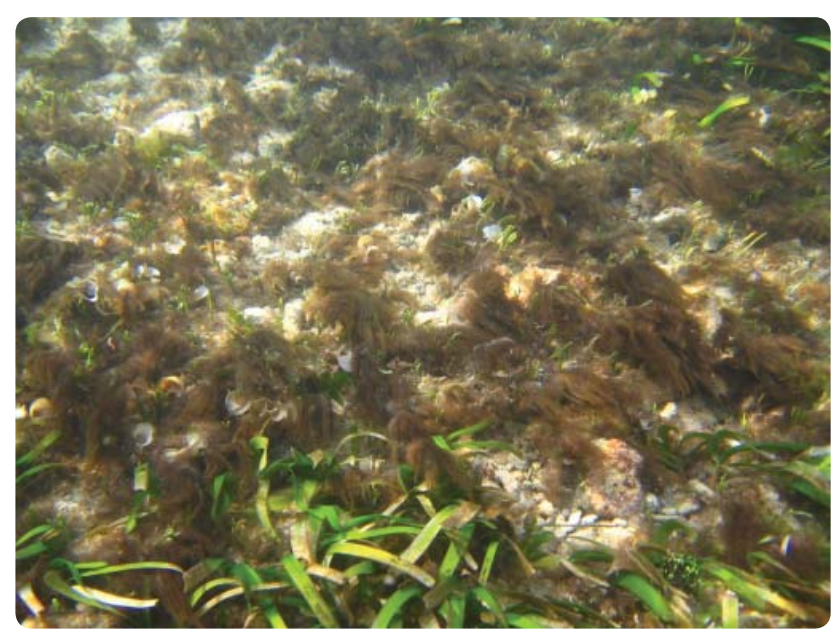

exception of the last category (four or greater). When stratified by age under 10 versus 10 and above, the dose response was similar (data not shown).

\section{Environmental investigation}

No obvious evidence of acute environmental contamination was found. The reef surrounding Murilo was in extremely poor condition, consisting of predominantly dead coral and large amounts of algae presumed to be Lyngbya majuscula (Figure 4).

No leftover turtle meat was found - the community consumed most of the turtle and discarded the remainder into the sea. However, the scutes and several bones were recovered. These were sent for laboratory analysis.

At least six dogs were reported to have died suddenly, presumably after eating turtle leftovers. All but two of these dogs were subsequently discarded or consumed (there were no reports of illness after consuming dogs). Of the remaining two, one was completely decomposed; the other, not completely decomposed, was dissected and stomach contents were sent for laboratory analysis.

\section{Laboratory testing}

All samples tested were below the limit of detection for all toxins tested.

\section{DISCUSSION}

This investigation shows strong epidemiologic evidence that chelonitoxism was the cause of mass illness on Murilo. The convincing dose-response relationship particularly supports that turtle was the cause of illness. We suspect that the dose-response failure of those who reported eating more than four servings of turtle was due to consumption of less turtle stew than reported.

Children are expected to be more severely affected by chelonitoxism due to their lower body mass and more sensitive immune systems. ${ }^{1}$ Therefore the death of several children in this outbreak was not unexpected. It is not clear why one child (Case 6) reported a nonserious illness only to die suddenly several days later. It is also not clear why the two adult males developed serious disease and died, although there was some evidence that they may have eaten larger amounts of turtle than the others before the feast. That they alone may have eaten raw turtle during preparation is likely not relevant as most chelonitoxins are heat-stable. ${ }^{3}$

Several infants developed illness without eating turtle but had been breastfed from a mother who ate turtle. Passage of chelonitoxins through breast milk has been documented. ${ }^{3}$ The toxins are thought to be virtually eliminated from the body within a few days, posing no ongoing threat for breastfeeding infants in Murilo. There is no other known transmission of these toxins from person to person.

The range of illness described in this investigation, from relatively mild mucus membrane irritation to coma and death, is consistent with previously reported cases of chelonitoxism. ${ }^{2}$ Despite intensive care, patients may succumb to the effects of the poison as there is no specific treatment for chelonitoxism. One possible toxin that may explain the widespread illness reported during this investigation is lyngbyatoxin $A$, the major toxin produced by Lyngbya majuscula. It has a direct inflammatory effect on tissue, ${ }^{4}$ which my have caused the sore throats, mouth pain and oral ulcers. Although there is little evidence of neurotoxicity of lyngbyatoxin, it is possible that large quantities may account for the neurologic toxicity seen in the fatal cases and as documented in other reports of severe chelonitoxism. ${ }^{2}$ 
Lyngbyatoxin A has been isolated from a green sea turtle (Chelonia mydas) associated with fatal chelonitoxism ${ }^{5}$ but not a hawksbill turtle. Although hawksbill turtles are carnivores, they also feed on sea grasses and algae, ${ }^{6}$ so algal toxins cannot be ruled out. The clinical picture of chelonitoxism caused by Chelonia mydas is the same as that caused by Eretmochelys imbricata, ${ }^{7,8}$ hence the toxin could have been either of these.

In this study, all human, animal and algal samples were negative for all toxins tested. Chelonitoxins have not been found previously in patient sera and only have been isolated from turtle meat, ${ }^{5}$ not scutes or bones, which were the only turtle tissues available here. Although the algae tested, which appeared to be Lyngbya majuscula, did not contain toxins, we cannot rule out the possibility that lyngbyatoxin was the etiology, as the turtle may have grazed from a different algal site. Alternatively, another toxin that was not tested for may have been the cause. It is possible that failure to detect toxins resulted from inadequate sampling or incomplete testing rather than true absence of toxins in the consumed turtle.

Hawksbill turtles have routinely been eaten on Murilo, yet there is no history of previous poisoning related to turtle consumption. It is not clear why the turtle eaten in this outbreak was toxic; however, most sea turtle species, particularly hawksbill turtles, can be poisonous if consumed. ${ }^{3}$ There is no reliable way to determine which individual turtles are poisonous, but it is more likely if they have eaten foods that may contain high levels of toxins. Foods high in toxins include bluegreen algae such as Lyngbya majuscula and also a variety of sponges and other marine organisms. Turtle eggs are also known to have high levels of a variety of toxins. ${ }^{1}$ There have been reports of other turtle poisonings in the Federated States of Micronesia including on Sapwuahfik, Pohnpei State, in April 2010 (D Buden, personal communication) and two previous incidents (including three deaths) on the same island in $1997 .^{9}$

As any turtle or turtle eggs may be toxic (i.e. Murilo is not unique), we recommended the cessation of consumption of turtle products throughout the Federated States of Micronesia. Even though sea turtles are relatively abundant in Chuuk, they are endangered throughout the world (hawksbill turtles are critically endangered); ${ }^{10}$ therefore, efforts to protect human health by reducing turtle capture have the added benefit of helping to conserve these rapidly disappearing species.
The devastating incident on Murilo highlights the dangers, particularly to children, of consuming turtle meat. Future incidents are certain to occur unless action is taken to alter turtle-eating behaviour in coastal communities throughout the world. Since all turtles and their eggs are capable of being toxic, we strongly recommend ceasing consumption of any turtles or their eggs to protect public health.

\section{Funding}

None.

\section{Conflicts of interest}

None declared.

\section{Acknowledgements}

We would like to acknowledge the assistance of the community of Murilo, the Chuuk Police Department, Department of Health Services, and Department of Marine Resources in our investigation. Particular thanks goes to Ismael Shapucy, Kachusy Farek, Pastor Mesenty, Dave Williander, Tafson Menesio, Riten Billias, Curtis Sos, Alex Narruhn and Benty Rickson. We also thank Forensic and Scientific Services, Queensland, Australia, for providing laboratory testing of samples. Finally, we acknowledge the clinical care and data provided by the late Herliep Nowell.

\section{References:}

1. Aguirre AA et al. Hazards associated with the consumption of sea turtle meat and eggs: A review for health care workers and the general public. EcoHealth, 2006, 3:141-153. doi:10.1007/ s10393-006-0032-x

2. Fussy $A$ et al. Chelonitoxism: new case reports in French Polynesia and review of the literature. Toxicon, 2007, 49:827-832. doi:10.1016/j.toxicon.2006.12.002 pmid:17250862

3. Limpus CJ. Sea turtles. In: Covacevich J, editor. Toxic Plants and Animals: A Guide for Australia. Brisbane, Queensland Museum, 1987:189-194.

4. Osborne NJT, Webb PM, Shaw GR. The toxins of Lyngbya majuscula and their human and ecological health effects. Environment International, 2001, 27:381-392. doi:10.1016/ S0160-4120(01)00098-8 pmid:11757852

5. Yasumoto T. Fish poisoning due to toxins of microalgal origins in the Pacific. Toxicon, 1998, 36:1515-1518. doi:10.1016/ S0041-0101(98)00142-1 pmid:9792166

6. Limpus CJ. Hawksbill turtle, Eretmochelys imbricata (Linnaeus). In: Fein L, editor. A biological review of Australian marine turtle species. Brisbane, Queensland Environmental Protection Agency, 2009: 1-53. 
7. Champetier de Ribes $\mathrm{G}$ et al. Un problème de santé réémergent à Madagascar: les intoxications collectives par consommation d'animaux marins. Aspects épidémiologiques, cliniques et toxicologiques des épisodes notifiés de janvier 1993 à janvier 1998 [In French]. Archives de I'Institut Pasteur de Madagascar, 1998, 64:71-76.

8. Champetier de Ribes $\mathrm{G}$ et al. Intoxications par animaux marins vénéneux à Madagascar (ichtyosarcotoxisme et chélonitoxisme): données épidémiologiques récentes [In French]. Bulletin de la Société de Pathologie Exotique, 2007, 90:268-272.

9. Buden DW. The reptiles of Sapwuahfik Atoll, Federated States of Micronesia. Micronesia, 2000, 32:245-56.

10. IUCN Red List of Threatened Species. Version 2014.3. Cambridge, United Kingdom, International Union of Conservation of Nature and Natual Resources, 2014 (http://www.iucnredlist. org, accessed 4 December 2014). 


\title{
Hospital preparedness for Ebola virus disease: a training course in the Philippines
}

\author{
Celia Carlos, ${ }^{a}$ Rowena Capistrano, ${ }^{a}$ Charissa Fay Tobora, ${ }^{a}$ Mari Rose delos Reyes, ${ }^{a}$ Socorro Lupisan, ${ }^{\circ}$

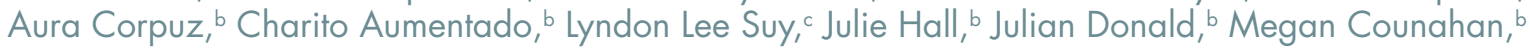 \\ Melanie S Curless, ${ }^{d}$ Wendy Rhymer, ${ }^{,}$Melanie Gavin, ${ }^{d}$ Chelsea Lynch, ${ }^{d}$ Meredith A Black, ${ }^{d}$ Albert D Anduyon, ${ }^{a}$ \\ Petra Buttner ${ }^{f}$ and Rick Speare ${ }^{f, g}$ \\ Correspondence to Rick Speare (e-mail: rickspeare@gmail.com).
}

Objective: To develop, teach and evaluate a training workshop that could rapidly prepare large numbers of health professionals working in hospitals in the Philippines to detect and safely manage Ebola virus disease (EVD). The strategy was to train teams (each usually with five members) of key health professionals from public, private and local government hospitals across the Philippines who could then guide Ebola preparedness in their hospitals.

Methods: The workshop was developed collaboratively by the Philippine Department of Health and the country office of the World Health Organization. It was evaluated using a pre- and post-workshop test and two evaluation forms. Chi-square tests and linear regression analyses were conducted comparing pre- and post-workshop test results.

Results: A three-day workshop was developed and used to train 364 doctors, nurses and medical technologists from 78 hospitals across the Philippines in three initial batches. Knowledge about EVD increased significantly $(P<0.009)$ although knowledge on transmission remained suboptimal. Confidence in managing EVD increased significantly $(P=0.018)$ with $96 \%$ of participants feeling more prepared to safely manage EVD cases.

Discussion: The three-day workshop to prepare hospital staff for EVD was effective at increasing the level of knowledge about EVD and the level of confidence in managing EVD safely. This workshop could be adapted for use as baseline training in EVD in other developing countries to prepare large numbers of hospital staff to rapidly detect, isolate and safely manage EVD cases.

$\mathrm{T}$ he West African Ebola virus disease (EVD) outbreak was declared a public health emergency of international concern (PHEIC) by the World Health Organization (WHO) on 8 August 2014. ${ }^{1}$ This outbreak is unprecedented with ongoing transmission in Guinea, Liberia and Sierra Leone and secondary localized outbreaks in Mali, Nigeria, Spain and the United States of America. Although estimates vary, the case fatality rate (CFR) is high in the three West African countries with ongoing transmission: cumulative CFR for the outbreak on 24 December 2014 was 70\% for cases with a recorded definitive outcome; 58-60\% for hospitalized patients; and 55\% (359/649) for healthcare workers (HCWs). ${ }^{2}$ Using the total figures for Guinea, Liberia and Sierra Leone gives a cumulative CFR of 39\% (7574/19 463) for all probable and confirmed cases, an underestimate of CFR since the fate of apparently more than 2000 cases are unknown. ${ }^{2}$ This EVD outbreak was the third PHEIC to be declared under the International Health Regulations (2005) (IHR). IHR is a legal framework that directs and governs its signatories' activities, aiming to protect the global community from public health risks and emergencies that cross international borders. ${ }^{3}$ At its core is the obligation for countries to develop, strengthen and maintain public health capacities for surveillance and response to be able to detect, assess, notify and report events and respond to a PHEIC. ${ }^{3}$

The West African EVD outbreak is considered such a threat to global security that for the first time the United Nations General Assembly, supported by the United Nations Security Council, mandated a United Nations Mission for Ebola Emergency Response for a public health event. ${ }^{4}$

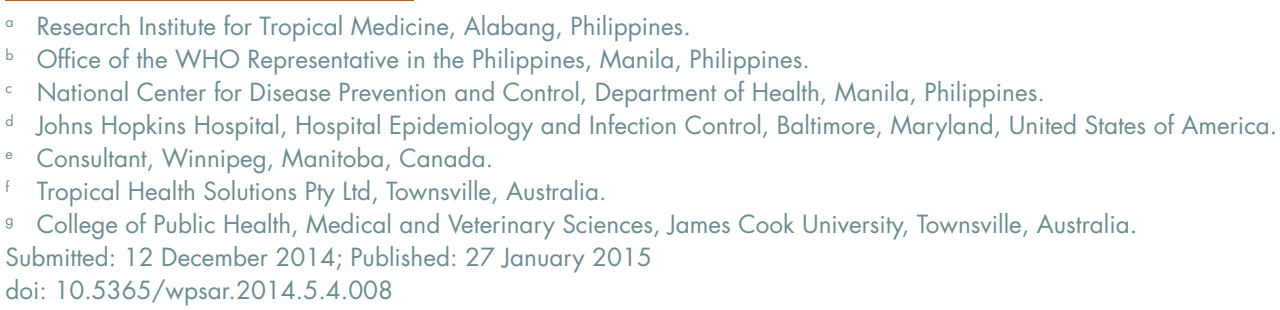


In 2013 the Philippines had 2.295 million formally registered Overseas Filipino Workers (OFW). ${ }^{5}$ Of these, $1700(<1 \%)$ were registered in Africa. However, the Commission on Filipinos Overseas estimated in 2012 there were 10.46 million Filipinos working overseas either permanently or temporarily. ${ }^{6}$ Their estimate in the Ebola-affected countries of Guinea, Liberia and Sierra Leone was $1212 .{ }^{6}$ It is traditional for OFW to return to the Philippines in high numbers at Christmas time. ${ }^{7}$

The Philippine Department of Health ( $\mathrm{DOH})$ is experienced with the management of outbreaks and has a relatively robust surveillance system with a history of managing imported emerging and re-emerging infectious diseases. ${ }^{8-10}$

It was against this backdrop of the risk of global transmission and returning OFW that the $\mathrm{DOH}$ asked the WHO country office in the Philippines for assistance in designing and implementing a training workshop to prepare government and private health practitioners in the management of EVD.

Countries unaffected by EVD, such as the Philippines, are required to prepare for the introduction of cases. Even if no EVD cases occur, symptomatic travellers from West Africa who meet the case definition of suspected EVD have to be managed according to the same protocols until they are confirmed as EVD negative. ${ }^{11}$ Although briefings for health care workers (HCWs) in Ebola treatment centres have been published, ${ }^{12-14}$ we were unable to locate a course designed to prepare clinicians for imported EVD in developing country settings.

The goal of the training was to increase capacity to rapidly detect, isolate and safely care for EVD cases within the Philippine health system, both public and private. The aims of the workshop were for participants to be able to provide safer care for patients with EVD and to prevent disease transmission in the health-care and community settings. The strategy was to train teams (each usually with five members) of key health professionals from public, private and local government hospitals across the Philippines who could then guide Ebola preparedness in their hospitals. The focus was on hospitals since it is highly likely that, if EVD occurs in the Philippines, patients will be identified and managed in hospitals. Although the DOH has appointed some hospitals to be EVD referral hospitals, the initial presentation of cases could occur at any hospital.
To ensure that participants followed official guidelines, the workshop was based on the DOH's Interim Guidelines for the Prevention and Management of Ebola Virus Disease (26 August 2014).

The aim of this paper is to describe the training programme and its evaluation.

\section{METHODS}

\section{Setting}

The curriculum and content were developed collaboratively by the Research Institute for Tropical Medicine (RITM, the $\mathrm{DOH}$ research institute for infectious diseases), the WHO country office and consultants employed by WHO as private individuals or from Johns Hopkins Hospital and Tropical Health Solutions. RITM, located in Metropolitan Manila, has a large training centre and staff experienced in running workshops. Training laboratories were available for practical sessions, and RITM's infection control team (experienced with SARS and other emerging infectious diseases) played a major role.

\section{Workshops}

Each workshop extended over three days and consisted of 18 lectures and 10 practical or small group sessions, including three practical sessions to don (put on) and doff (take off) personal protective equipment (PPE) (Table 1). ${ }^{15}$ Everyone participated in at least two PPE sessions. At registration, every participant was given one set of PPE (apart from rubber boots) for personal use during the workshop. The PPE sessions were conducted in large groups numbering from 50 to 120 (Figure 1). PPE donning and doffing skills were first demonstrated to the whole group, which was then divided into two groups for demonstration and practice. Rigid donning and doffing protocols were followed and overseen by participants acting as trained observers supervised by course facilitators. In the final PPE session, red water-based paint was applied to the PPE to simulate contamination by body fluids, adding a sense of realism to the doffing process (Figure 2).

A specialized series of three lectures and a practical session were run separately for medical technologists (Table 1). In the Philippines, medical technologists have a Bachelor of Science in Medical Technology and are equivalent to laboratory scientists in other countries. The 


\section{Table 1. Structure of workshop on hospital management of EVD*}

\begin{tabular}{|c|c|c|}
\hline Session & Type of activity & Materials used \\
\hline \multicolumn{3}{|l|}{ Day 1} \\
\hline Opening & $\begin{array}{l}\text { Formal opening with support from } \\
\text { WHO country office and DOH }\end{array}$ & \\
\hline Introduction & Lecture & \\
\hline $\begin{array}{l}\text { Ebola - basics, natural history and epidemiology of the } \\
\text { West African outbreak; Reston Ebolavirus in the Philippines }\end{array}$ & Lectures & \\
\hline Screening and triage & Lecture; small group work on six cases & $\begin{array}{l}\text { Participants' sheet of cases } \\
\text { Facilitators' guide } \\
\text { Appendices B.2 and B. } 3 \text { of the DOH } \\
\text { Interim Guidelines }\end{array}$ \\
\hline Treatment and discharge & Lecture & \\
\hline Laboratory support and biosafety & Lecture & \\
\hline Laboratory confirmation of EVD & Lecture & \\
\hline Infection control for EVD & $\begin{array}{l}\text { Lecture } \\
\text { Practical session on removing gloves }\end{array}$ & $\begin{array}{l}\text { Individual gloves and alcohol-based hand } \\
\text { rub } \\
\text { Glow-powder and UV lights }\end{array}$ \\
\hline Ethical issues about clinical activities in EVD patients & Group discussion & \\
\hline PPE for EVD: donning and doffing & $\begin{array}{l}\text { Demonstration } \\
\text { Practical sessions }\end{array}$ & $\begin{array}{l}\text { Donning and doffing schedules } \\
\text { Facilitators' guide } \\
\text { Individual PPE }\end{array}$ \\
\hline \multicolumn{3}{|l|}{ Day 2} \\
\hline Isolation and patient flow & Lecture & \\
\hline Designing isolation units for your hospital & Practical session & Plans of each participant's hospital \\
\hline $\begin{array}{l}\text { Management of sharps and post-exposure management for } \\
\text { EVD }\end{array}$ & $\begin{array}{l}\text { Lecture } \\
\text { Role plays }\end{array}$ & \\
\hline Environmental cleaning and waste management for EVD & Lecture & \\
\hline Transport of EVD patients & Lecture & \\
\hline PPE for EVD: donning and doffing & Practical sessions & $\begin{array}{l}\text { Donning and doffing schedules } \\
\text { Facilitators' guide } \\
\text { Individual PPE }\end{array}$ \\
\hline \multicolumn{3}{|l|}{ Day 3} \\
\hline Comment on plans for isolation units & $\begin{array}{l}\text { Commentary of each group's plans for an } \\
\text { isolation unit in their hospital }\end{array}$ & $\begin{array}{l}\text { Photographs of individual hospital isolation } \\
\text { plans in PowerPoint presentation }\end{array}$ \\
\hline Community health and support & Lecture & \\
\hline Staff health and support in EVD & Lecture & \\
\hline Safe and dignified burial for EVD & Lecture & \\
\hline Epidemic management and surveillance & Lecture & \\
\hline Contact tracing & Lecture & \\
\hline Role of subnational laboratories & Lecture & \\
\hline $\begin{array}{l}\text { Question and answer session relevant to hospital } \\
\text { management of EVD }\end{array}$ & Interactive session with $\mathrm{DOH}$ representative & \\
\hline PPE for EVD: donning and doffing & $\begin{array}{l}\text { Practical session with addition of red water- } \\
\text { based paint used to contaminate PPE }\end{array}$ & $\begin{array}{l}\text { Donning and doffing schedules } \\
\text { Facilitators' guide } \\
\text { Individual PPE } \\
\text { Water-based paint }\end{array}$ \\
\hline \multicolumn{3}{|l|}{ Additional specialized workshop for medical technologists } \\
\hline EVD risk assessment and biosafety for laboratory personnel & Lecture & \\
\hline Referral system, transport and storage of EVD specimens & Lecture & \\
\hline $\begin{array}{l}\text { Laboratory waste management, decontamination and } \\
\text { laboratory emergencies for EVD }\end{array}$ & Lecture & \\
\hline Laboratory procedures with PPE for EVD & $\begin{array}{l}\text { Practical sessions: blood collection, } \\
\text { specimen processing; packaging for } \\
\text { transport }\end{array}$ & $\begin{array}{l}\text { Individual PPE } \\
\text { Blood collection equipment } \\
\text { Safety cabinet } \\
\text { Packaging and transportation materials }\end{array}$ \\
\hline Closing ceremony & $\begin{array}{l}\text { Speeches by WHO country office and DOH; } \\
\text { presentation of certificates }\end{array}$ & Certificates of participation \\
\hline
\end{tabular}

* Modules and guide are available at http://www.wpro.who.int/philippines/mediacentre/features/ebolatraining_materials/en/.

DOH, Department of Health; EVD, Ebola virus disease; PPE, personal protective equipment; UV, ultraviolet rays; WHO, World Health Organization. 
Figure 1. Donning and doffing PPE during the Philippine DOH Ebola preparedness workshop*

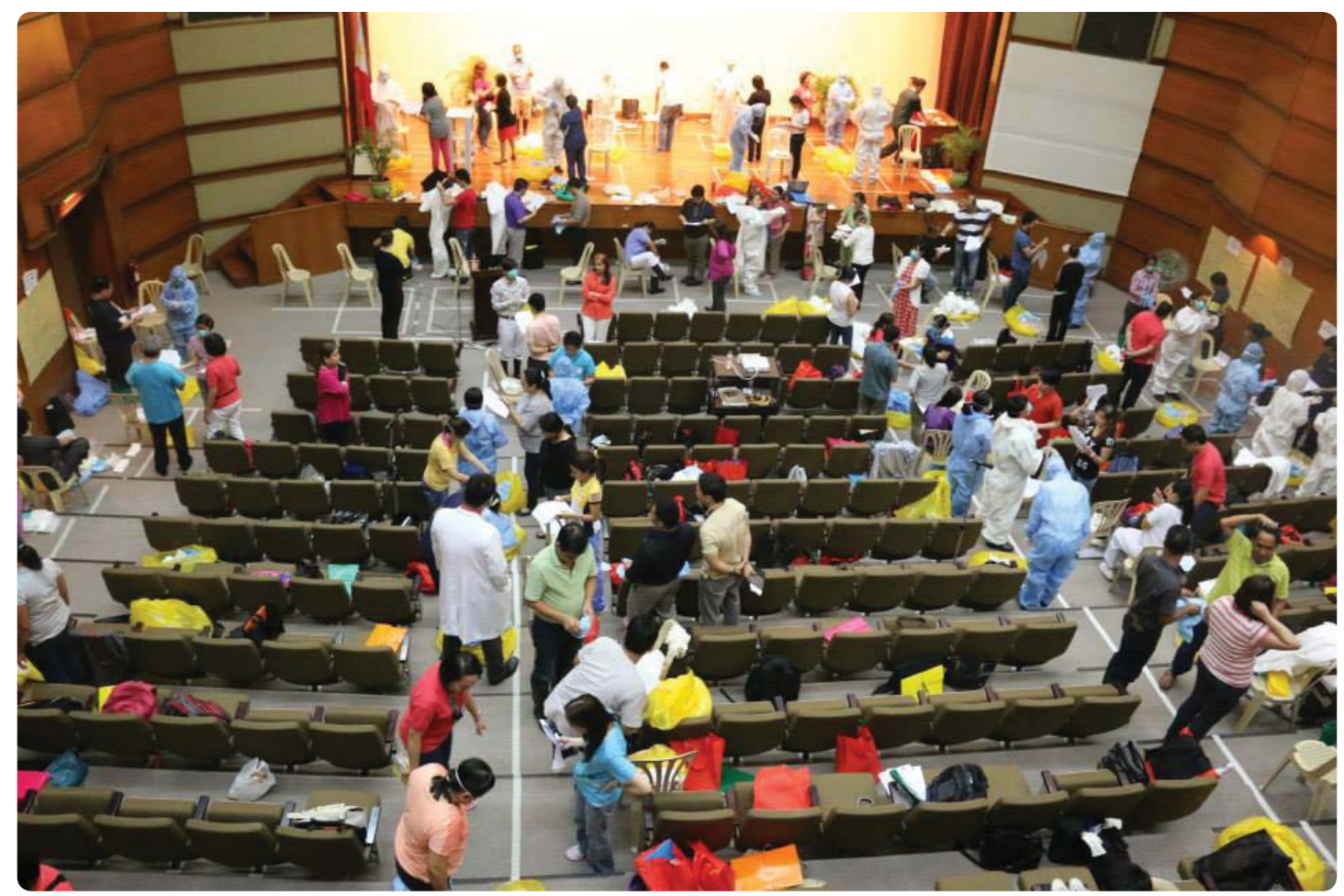

Source: Photo taken by the Research Institute for Tropical Disease photographer.

* Each health-care provider worked with a trained observer. Facilitators supervised groups of participants. Tape on the floor helped simulate workflow by identifying low and high risk areas.

medical technologists' sessions included opportunities to practise venepuncture and use laboratory equipment while wearing EVD PPE. At the closing session on Day 3, the Director of RITM and the WHO Representative or their delegates gave closing speeches and presented certificates of participation.

\section{Participants}

Since the $\mathrm{DOH}$ wished to rapidly and extensively distribute EVD knowledge and skills, three initial workshops were held from 28 October to 13 November 2014 with large numbers of participants in each. Hospitals selected by the $\mathrm{DOH}$ from each province were asked to send a team of five including at least one doctor, one nurse and one medical technologist. Batches 1, 2 and 3 had 127, 115 and 122 participants, respectively, from 78 hospitals (21 regional hospitals, 22 private hospitals and 35 local government hospitals).

The training programme for each batch of trainees was modified as new evidence was reported in the
Figure 2. Water-based red paint has been applied to a participant's PPE to simulate extensive contamination by blood*

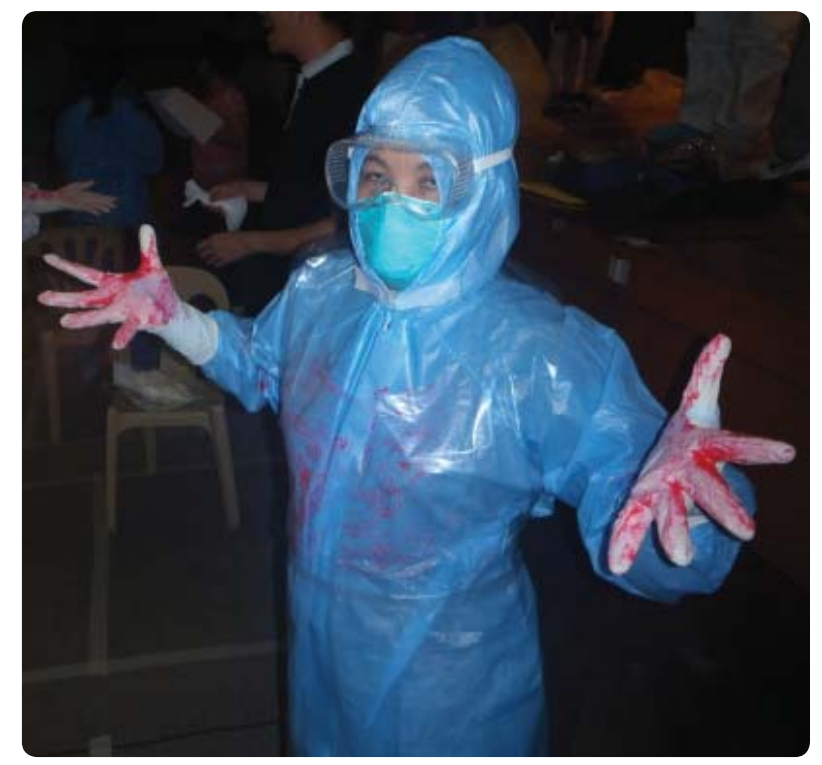

Source: Photo taken by Rick Speare.

* Gloves, gown and the clear, disposable apron are contaminated. 
literature or online by the Centers for Disease Control and Prevention (CDC) and WHO. ${ }^{16,17}$

\section{Evaluation}

Three tools were used to evaluate the workshop: a preand post-workshop test, two evaluation forms postworkshop and One Minute Reflections $(\mathrm{OMR})^{18}$ on Days 1 and 2. The test was developed by WHO consultants and piloted on RITM medical and nursing staff; the RITM evaluation form is used routinely for all workshops, and the EVD-specific evaluation form was a modification of the Johns Hopkins Hospital form used for EVD training workshops. All questionnaires were anonymous.

Effect of the workshop on knowledge was tested using a pre- and post-workshop test administered on Day 1 before the lectures commenced and on Day 3 before the final practical session on PPE. Knowledge was assessed by 10 questions. Confidence in being able to manage EVD was assessed on a five-point Likert scale (strongly disagree, disagree, neither disagree or agree, agree and strongly agree) in response to the statement: "I am confident that I can be safe when caring for a patient with Ebola virus disease." The answers were summarized and presented to the participants before the closing session on Day 3.

At the end of activities on Days 1 and 2 participants were asked to complete the OMR which asked two questions: (1) What was the most useful, meaningful or intriguing thing/s that you learnt during this day? and (2) what question/s remains uppermost in your mind as we end this day? The responses from the OMR were addressed in the first sessions on Days 2 and 3.

\section{Statistical analysis}

Pre- and post-workshop test: If a response to one of the 10 knowledge questions was missing, it was set to being incorrect. The number of missing values per question ranged between 0 and 4 for the pre-workshop test and between 0 and 6 for the post-workshop test. The 10 knowledge questions were recoded to 0 for "incorrect" and 1 for "correct" and added up to get the overall number of correct answers. This outcome variable was logarithmically transformed to achieve approximate normal distribution for the linear regression analysis. Pre- and post-workshop participants were analysed as unpaired data because questionnaires could not be linked.

Categorical characteristics are described using absolute and relative frequencies. Age was described using mean value and standard deviation. The number of correct answers given to the 10 knowledge questions was described using median value and inter-quartile range (IQR). Confidence intervals of $95 \%$ were calculated for correct answers to knowledge questions; pre- and postworkshop test results were compared using chi-square tests. The overall logarithmically transformed number of correct answers pre- and post-training were compared using linear regression. Linear regression analysis was conducted to analyse whether profession, age and gender showed independent effects on the logarithmically transformed number of correct answers post-workshop. Profession and gender were dummy coded for this analysis, while age was initially considered, continuous and was then categorized using the quartiles and dummy coded. Chi-square tests were used to assess effects of age, gender and profession on level of confidence dealing with an Ebola case post-workshop. All analysis was adjusted for the cluster effect of the three workshops. Analysis was conducted using STATA, release 12.1 (StataCorp LP, College Station, Texas). Since this was an operational evaluation and findings were progressively used to improve the workshop, ethics approval was not sought.

\section{RESULTS}

\section{Participants}

A total of 285 participants ( $78.3 \%$ of all participants) and 364 participants (100\% of all participants) completed the pre- and post-workshop tests, respectively. For the pre- and post-workshop tests, respectively, participants were doctors (33.3\% and $35.0 \%$ ), nurses $(42.9 \%$ and $40.0 \%$ ), medical technologists ( $20.3 \%$ and $20.1 \%$ ) and others (3.4\% and $4.5 \%$ ). Participants who completed the pre- and post-training participants were predominantly female (59.6\% and $61.9 \%$, respectively) and mean ages and ranges were the same at 38.2 (21 to 62 years).

\section{Improvement in knowledge}

Of the 10 knowledge questions, three were correctly answered by more than $90 \%$ of pre-workshop test 
Table 2. Percentage and $95 \%$ confidence interval of correct responses to 10 knowledge questions pre- and post-workshop*

\begin{tabular}{|c|c|c|c|c|}
\hline Knowledge questions & $\begin{array}{l}\text { Pre-workshop } \\
\quad(n=285) \\
\%[95 \% \mathrm{Cl}]^{\dagger}\end{array}$ & $\begin{array}{l}\text { Post-workshop } \\
(n=364) \\
\%[95 \% \mathrm{Cl}]^{\dagger}\end{array}$ & $\%$ change & $p$-value \\
\hline $\begin{array}{l}\text { 1. The maximum incubation period of Ebola virus disease } \\
\text { (EVD) is } 42 \text { days (False) }\end{array}$ & $80.0 \% ;[62.4,97.6]^{*}$ & $90.1 \% ;[77.8,100]$ & 10.1 & 0.254 \\
\hline $\begin{array}{l}\text { 2. A person infected with Ebola virus can pass on the } \\
\text { virus before symptoms begin (False) }\end{array}$ & $55.8 \% ;[26.1,85.5]$ & $89.0 \% ;[73.8,100]$ & 33.2 & 0.018 \\
\hline $\begin{array}{l}\text { 3. Multiple choice question on transmission routes for } \\
\text { EVD }\end{array}$ & $34.0 \% ;[11.3,56.7]$ & $59.1 \% ;[44.6,73.5]$ & 25.1 & 0.055 \\
\hline $\begin{array}{l}\text { 4. Soap and water is an effective method of hand hygiene } \\
\text { when caring for patients with EVD (True) }\end{array}$ & $80.4 \% ;[70.8,89.9]$ & $63.5 \% ;[33.6,93.3]$ & -16.9 & 0.081 \\
\hline $\begin{array}{l}\text { 5. While working in personal protective equipment, } \\
\text { health-care workers caring for patients with EVD } \\
\text { should have no skin exposed (True) }\end{array}$ & $96.8 \% ;[94.0,99.7]$ & $98.9 \% ;[96.7,100]$ & 2.1 & 0.073 \\
\hline $\begin{array}{l}\text { 6. The World Health Organization recommends double } \\
\text { gloves when caring for a suspect or confirmed Ebola } \\
\text { patient (True) }\end{array}$ & $85.3 \% ;[71.7,98.8]$ & $98.4 \% ;[96.2,100]$ & 13.1 & 0.007 \\
\hline $\begin{array}{l}\text { 7. Multiple choice question on the correct proportions to } \\
\text { make up } 0.5 \% \text { bleach disinfectant }\end{array}$ & $19.6 \% ;[12.8,26.5]$ & $86.5 \% ;[65.0,100]$ & 66.9 & 0.009 \\
\hline $\begin{array}{l}\text { 8. Waste from an Ebola patient in a typical Philippine } \\
\text { hospital can be discarded as is usual (False) }\end{array}$ & $95.1 \% ;[91.1,99.1]$ & $96.7 \% ;[96.3,97.1]$ & 1.6 & 0.161 \\
\hline $\begin{array}{l}\text { 9. When caring for a suspected or confirmed Ebola } \\
\text { patient, do not perform any blood tests except an } \\
\text { Ebola test (False) }\end{array}$ & $76.1 \% ;[60.7,91.6]$ & $75.0 \% ;[47.2,100]$ & -1.1 & 0.719 \\
\hline 10. A person dead from EBV can be embalmed (False) & $90.5 \% ;[80.8,100]$ & 97.3\%; [91.8, 100] & 6.8 & 0.188 \\
\hline
\end{tabular}

participant (no skin exposed in EVD PPE, EVD waste handled differently, no embalming with EVD) (Table 2). For the other seven questions, pre-workshop knowledge was poor $(<50 \%$ correct) for two questions (transmission routes of EVD, correct mixing of $0.5 \%$ bleach). The percentage of correct answers rose by $10 \%$ or more in the post-workshop test for five questions (three of them statistically significant), remained largely unchanged for four (three of which had more than 90\% correct in pretest) and one fell by 16.9\% (soap and water in EVD) but this did not achieve statistical significance (Table 2).

The percentage of participants who correctly answered all 10 questions was $2.8 \%$ (8 of 285) and $22.5 \%$ (82 to 364 ) pre- and post-workshop, respectively. The number of questions correctly answered by participants rose from a pre-workshop median of seven (IQR $=6$ to 8 ; range 3 to 10 ) to a post-workshop median of nine $(I Q R=8$ to 9 ; range 4 to 10$)(P<0.009)$ (Figure 3).
Figure 3. Percentage of knowledge questions correctly answered pre- and post-workshop

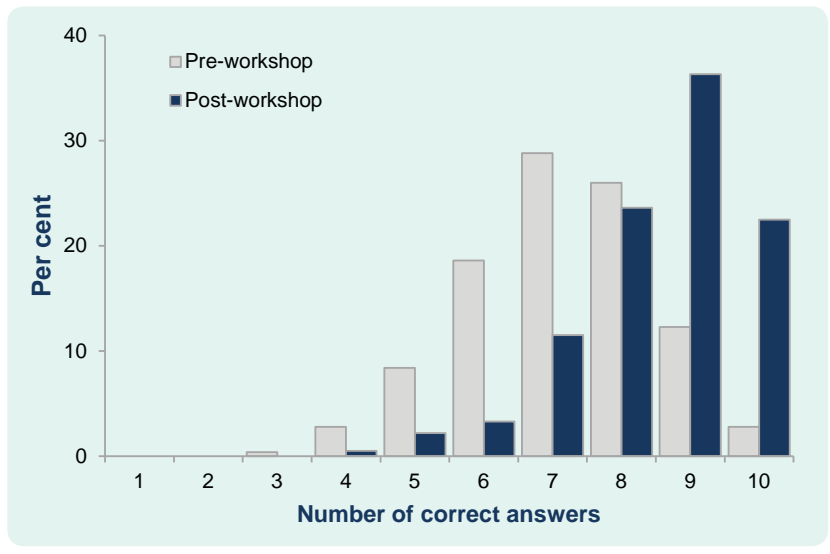

Profession (nurse $P=0.775$, medical technologist $P=0.431$, other $P=0.335$, doctor $=$ reference group), age (30-39 years $P=0.271 ; 40-44$ years $P=0.273$; $\geq 45$ years $P=0.728$; reference $<30$ years) and gender $(P=0.071)$ showed no significant independent 
Table 3. Associations between number of correct responses and level of confidence post-workshop and age, gender and profession

\begin{tabular}{|c|c|c|c|c|}
\hline Influencing characteristics & $\begin{array}{l}\text { Median number of } \\
\text { correct responses } \\
\text { post-workshop [IQR] }\end{array}$ & $p$-value* & $\begin{array}{l}\% \text { agreeing or strongly agreeing with } \\
\text { "I am confident that I can be safe when } \\
\text { caring for a patient with Ebola virus } \\
\text { disease" post-workshop }\end{array}$ & $p$-value ${ }^{\dagger}$ \\
\hline \multicolumn{5}{|l|}{ Profession } \\
\hline Doctor & $9[8,9]$ & Reference & 91.7 & \multirow{4}{*}{0.458} \\
\hline Nurse & $9[8,10]$ & 0.775 & 86.6 & \\
\hline Laboratory worker & $8[8,9]$ & 0.431 & 84.7 & \\
\hline Other & $9[6.75,9]$ & 0.335 & 78.6 & \\
\hline \multicolumn{5}{|l|}{ Gender } \\
\hline Female & $9[8,10]$ & Reference & 86.7 & \multirow{2}{*}{0.404} \\
\hline Male & $9[8,9]$ & 0.071 & 91.3 & \\
\hline \multicolumn{5}{|l|}{ Age } \\
\hline $0-29$ years & $9[8,9]$ & Reference & 91.5 & \multirow{4}{*}{0.412} \\
\hline 30-39 years & $9[8,10]$ & 0.271 & 91.2 & \\
\hline 40-44 years & $9[8,9]$ & 0.273 & 88.2 & \\
\hline 45 years and above & $9[8,10]$ & 0.728 & 80.8 & \\
\hline
\end{tabular}

* $p$-values are results of a linear regression model for post-workshop outcome including profession, age and gender as independent variables. $p$-values are results of chi-square tests. $P$-values are adjusted for cluster-effect.

$I Q R$, inter-quartile range.

Table 4. Evaluation of the EVD workshop using the general RITM form $(n=328)$

\begin{tabular}{lccccc}
\hline Section & Poor & Acceptable & Satisfactory & Very good & Excellent \\
\hline Content of lectures & $0.0 \%$ & $0.0 \%$ & $2.1 \%$ & $34.8 \%$ & $63.1 \%$ \\
Lecturers/presentations & $0.0 \%$ & $0.3 \%$ & $4.6 \%$ & $42.7 \%$ & $52.4 \%$ \\
Overall rating & $0.0 \%$ & $0.4 \%$ & $4.7 \%$ & $53.3 \%$ & $41.6 \%$ \\
\hline
\end{tabular}

effects on the number of correct answers post-workshop Evaluations

(Table 3).

\section{Improvement in confidence}

Participants' level of confidence in safely caring for an EVD patient rose markedly by the end of the workshop $(P=0.018)$. Pre-workshop, $27.3 \%$ disagreed or strongly disagreed with the statement: "I am confident that I can be safe when caring for a patient with Ebola virus disease," while post-training this percentage fell to $2.6 \%(P=0.018)$. On the other hand, pre-workshop, $32.5 \%$ of participants agreed or strongly agreed with the statement; post-training this percentage had risen to $87.2 \%$. The post-workshop level of confidence was not influenced by age $(P=0.412)$, gender $(P=0.404)$ or profession $(P=0.458)$ (Table 3 ).
Ratings in both evaluation forms were at the high end of a five-point Likert scale (Tables 4 and 5 ). The overall rating for the workshop was excellent $(72.2 \%)$, good (26.7\%), fair $(0.6 \%)$ and poor (0\%) (Table 4$)$. To the question "Do you feel you are more prepared to screen for and manage patients with Ebola?" 96.4\% of participants answered "Yes" (Table 5).

The OMR provided a useful means of addressing questions that were left unanswered after Days 1 and 2 . These questions fell mainly into two areas: knowledge and support. The former category consisted largely of questions about the disease. The latter category included detailed questions about how things would be done if an EVD case occurred; where the specialized items of PPE 
Table 5. Ratings on EVD-specific evaluation form $(n=333)$

\begin{tabular}{|c|c|c|c|c|c|}
\hline Section & $\begin{array}{l}\text { Strongly } \\
\text { disagree }\end{array}$ & Disagree & $\begin{array}{l}\text { Neither agree } \\
\text { or disagree }\end{array}$ & Agree & $\begin{array}{l}\text { Strongly } \\
\text { agree }\end{array}$ \\
\hline \multicolumn{6}{|l|}{ Course objectives and content } \\
\hline $\begin{array}{l}\text { I understood what the training was designed to } \\
\text { achieve. }\end{array}$ & $0.0 \%$ & $0.0 \%$ & $1.8 \%$ & $37.8 \%$ & $60.1 \%$ \\
\hline I feel like the training objectives were achieved. & $0.0 \%$ & $0.3 \%$ & $3.3 \%$ & $43.1 \%$ & $53.3 \%$ \\
\hline I know how to prepare for Ebola at my facility. & $0.0 \%$ & $0.6 \%$ & $9.8 \%$ & $53.4 \%$ & $36.2 \%$ \\
\hline $\begin{array}{l}\text { I understand infection control in management of } \\
\text { Ebola patients. }\end{array}$ & $0.0 \%$ & $0.3 \%$ & $3.8 \%$ & $49.1 \%$ & $46.7 \%$ \\
\hline $\begin{array}{l}\text { I feel confident applying infection control in } \\
\text { managing Ebola patients. }\end{array}$ & $0.0 \%$ & $1.2 \%$ & $11.5 \%$ & $58.0 \%$ & $29.0 \%$ \\
\hline $\begin{array}{l}\text { I know how to screen and triage potential Ebola } \\
\text { patients. }\end{array}$ & $0.0 \%$ & $0.3 \%$ & $3.9 \%$ & $48.5 \%$ & $47.3 \%$ \\
\hline \multicolumn{6}{|l|}{ Training materials } \\
\hline $\begin{array}{l}\text { I found the training materials for this workshop } \\
\text { to be well organized. }\end{array}$ & $0.0 \%$ & $1.8 \%$ & $2.4 \%$ & $46.7 \%$ & $49.1 \%$ \\
\hline $\begin{array}{l}\text { I think the training materials will be useful in the } \\
\text { future. }\end{array}$ & $0.0 \%$ & $1.5 \%$ & $8.0 \%$ & $46.3 \%$ & $43.7 \%$ \\
\hline \multicolumn{6}{|l|}{ Trainers' skills } \\
\hline $\begin{array}{l}\text { The trainers stated all session objectives clearly } \\
\text { and they were easy to follow. }\end{array}$ & $0.0 \%$ & $0.6 \%$ & $2.9 \%$ & $48.4 \%$ & $47.8 \%$ \\
\hline $\begin{array}{l}\text { The trainers were able to assist participants } \\
\text { when questions or difficulties arose. }\end{array}$ & $0.0 \%$ & $0.0 \%$ & $4.4 \%$ & $47.2 \%$ & $48.4 \%$ \\
\hline $\begin{array}{l}\text { The trainers were knowledgeable about the } \\
\text { topic. }\end{array}$ & $0.0 \%$ & $0.6 \%$ & $1.8 \%$ & $39.9 \%$ & $57.7 \%$ \\
\hline \multicolumn{6}{|l|}{ Course administration } \\
\hline The training schedule was acceptable. & $0.0 \%$ & $0.9 \%$ & $6.5 \%$ & $48.7 \%$ & $43.9 \%$ \\
\hline The training was well organized. & $0.0 \%$ & $0.9 \%$ & $6.5 \%$ & $50.4 \%$ & $42.1 \%$ \\
\hline
\end{tabular}

could be obtained; funding for preparedness; and the roles of institutions, DOH and other government bodies. Questions on knowledge were answered by lecturers and RITM staff. On the last day, the Director of the Emerging Infectious Disease Control Programme was on hand to answer operational questions. His attendance was very important because the policy situation is dynamic and evolving.

\section{DISCUSSION}

This three day workshop on hospital preparedness for EVD raised knowledge and increased the confidence of participants. Two areas of knowledge that were not as well understood that soap and water is effective for hand hygiene in EVD and the transmission of Ebola virus. The change in the former is understandable since during the practical sessions alcohol-based hand rubs were used for hand hygiene practice and to decontaminate gloves between doffing steps. Soap and water is recommended by WHO for hand hygiene for EVD if alcohol-based hand rubs are unavailable and as the preferred method if hands are visibly soiled. ${ }^{19}$ However, in many resourcelimited settings sinks may not be available at point of care or may not have adequate soap or hand drying materials. ${ }^{20}$ This has been identified as a current problem in Liberian hospitals. ${ }^{21,22}$ In the workshop, emphasizing the indications for soap and water may have increased the correct answers.

Epidemiological studies in African communities have shown that direct contact with a symptomatic case of EVD is required for transmission. ${ }^{23-25}$ Interestingly, although an early study showed no transmission in the community without direct contact, one of the primary cases acquired EVD while visiting the local hospital in Sudan with no identified contact with a hospitalized EVD case. ${ }^{23}$ Conveying how EVD transmits in the health care setting remains a difficult issue. ${ }^{26,27}$ When two nurses in the USA acquired EVD while wearing PPE with no apparent breaches in protocol, ${ }^{28}$ standards of PPE for EVD were upgraded by both $C D C$ and 
WHO. ${ }^{16,17}$ In hospitals and EVD isolation units the concept of "direct transmission" is confusing when it also includes transmission by needlestick, droplets and splashes and when aerosol transmission associated with clinical procedures (i.e. intubation) must be regarded as a risk. ${ }^{26}$ Although the correct answers for the question on transmission increased, it remained unacceptably low at $59.1 \%$. We need to develop a new approach for conveying the complexities of transmission routes in the health-care setting.

The increase in level of confidence was verified by the pre- and post-workshop test and by two questions in the EVD-specific evaluation form. Improvement in the knowledge of participants, particularly in the practice of proper donning and doffing of PPE, could have contributed to this. In the OMR, participants often mentioned PPE as the most useful thing learnt. This workshop appeared to be effective in providing baseline training to raise awareness in large numbers of HCWs.

Screening and triage procedures were emphasized in lectures and reinforced by a practical session using the DOH screening and triage forms on six cases, illustrating the importance of a detailed travel, contact and illness history in the non-Ebola setting. Failure to screen and triage patients in the hospital setting in Liberia has led to infection of HCWs. ${ }^{21,29}$ Screening and triage are essential strategies to rapidly detect EVD patients in countries not affected by EVD and to prevent transmission.

We could find no specific guidelines for training $\mathrm{HCWs}$ in non-Ebola countries to be prepared to manage EVD. The approach we used trained teams of key health professionals from hospitals across the Philippines in the basics with the aim that they would train others when they returned home. In developed countries the focus appears to be on major hospitals, some designated for high consequence pathogens such as Ebola, to do intensive preparatory training. ${ }^{30-32}$ The former approach may be more suitable in countries with limited resources. In these settings, non-specialized clinicians will be expected to participate in the management of EVD patients. Providing basic training for large groups of HCWs may best protect the majority of HCWs. Once a case of EVD is identified, more intensive training can be provided to those HCWs managing the case to quickly build on the basic knowledge and skills. Although knowledge may not always be retained over time, brief educational intervention of eight hours duration for disaster preparedness was proven to be effective and to have a long-term impact on nurses' knowledge. ${ }^{33}$

Limitations include use of the same test pre- and post-workshop, meaning increases in knowledge may be test- rather than disease-specific; and the $27.7 \%$ increase in responses for the post-workshop test which may breach the assumption that the pre- and postworkshop populations were the same. However, the professional, age and gender compositions of the samples were similar.

Although the context of the training was specific to the Philippines, the workshop could be adapted to other countries by substituting the Philippine $\mathrm{DOH}$ Interim Guidelines for EVD with each country's own guidelines and omitting the lecture on Ebola Reston since this species of Ebola virus is endemic only in the Philippines and China. ${ }^{34,35}$ Mention of Ebola Reston in the background lecture on Ebola would be adequate for other developing countries.

\section{CONCLUSIONS}

The three-day workshop developed by the Philippine DOH to prepare hospital staff for EVD was effective at increasing the level of knowledge about EVD and the level of confidence in managing EVD safely. In an Ebola outbreak, additional specialized training in use of PPE would be needed for those caring for EVD patients in hospital to reinforce the baseline training. This workshop could be adapted for use in other developing countries preparing their hospital staff to rapidly detect, isolate and safely manage EVD cases.

\section{Conflicts of interest}

None declared.

\section{Funding}

Development and evaluation of the workshop and employment of WHO consultants (Speare, Rhymer, Curless, Lynch, Gavin and Black) was funded by the Department for International Development. Philippine 
DOH provided funds to conduct the workshop. All staff of RITM, DOH and WHO were funded by their respective employers under routine funding.

\section{Acknowledgement}

We thank a large number of staff of RITM for logistic support in developing and conducting workshops and the IT staff of RITM in particular for assistance in entering data from evaluation forms.

\section{References:}

1. Statement on the first meeting of the IHR Emergency Committee on the 2014 Ebola outbreak in West Africa. Geneva, World Health Organization, 8 August 2014 (http://www.who.int/media centre/news/statements/2014/ebola-20140808/en/, accessed 25 November 2014).

2. Ebola Response Roadmap Situation Report 24 December 2014. Geneva, World Health Organization, 2014 (http://apps.who.int/ iris/bitstream/10665/146311/1/roadmapsitrep_24Dec14_eng. pdf?ua=1, accessed 30 December 2014).

3. International Health Regulations (2005) Second edition. Geneva, World Health Organization, 2008 (http://whqlibdoc. who.int/publications/2008/9789241580410_eng.pdf, accessed 24 November 2014).

4. Resolution adopted by the General Assembly on 19 September: 69/1. Measures to contain and combat the recent Ebola outbreak in West Africa. New York, United Nations General Assembly, 2014 (http://www.un.org/en/ga/search/view_doc.asp?symbol=A/ RES/69/1, accessed 25 November 2014).

5. Statistical Tables on Overseas Filipino Workers (OFW): 2013. Manila, National Statistics Office, 2014 (http://census.gov.ph/ content/statistical-tables-overseas-filipino-workers-ofw-2013, accessed 25 November 2014).

6. Stock estimate of Filipinos overseas as of December 2012. Manila, Commission on Filipinos Overseas, 2013 (http://cfo. gov.ph/images/stories/pdf/StockEstimate2012.pdf, accessed 26 November 2014).

7. Anonymous. Overseas Filipino Workers. Manila, Wikipilipinas, 2008 (http://en.wikipilipinas.org/index.php/Overseas_Filipino_ Workers, accessed 21 December 2014).

8. Pamaran RR et al. Epidemiological characterization of influenza A(H1N1)pdm09 cases from 2009 to 2010 in Baguio City, the Philippines. PLoS One, 2013, 11;8(11):e79916. doi:10.1371/ journal.pone.0079916 pmid:24244578

9. Anonymous. SARS outbreak in the Philippines. Weekly Epidemiological Record, 2003, 78:189-192. pmid:12836452

10. Corpuz A, Banatin CA. Surveillance in Post Extreme Emergencies and Disasters (SPEED). Early Warning Alert and Response Network Humanitarian Emergency Conference, Geneva, 17-19 March 2014.

11. Ebola and Marburg virus disease epidemics: preparedness, alert, control, and evaluation - Interim version 1.1. Geneva, World Health Organization, 2014 (http://www.who.int/csr/ disease/ebola/PACE_outbreaks_ebola_marburg_en.pdf, accessed 26 November 2014).

12. Med Box. Ebola toolbox. Wursburg, Department of Humanitarian Collaboration Medical Missions Institute, Advisory Organization for International Health (http://www.medbox.org/ebola-trainingmaterial/listing, accessed 20 November 2014).

13. Ebola ebriefing. Barcelona, Médecins Sans Frontières, 2014 (http://ecampus.msf.org/moodlemsf/mod/page/view.php?id = 22246, accessed 26 November 2014).

14. 2014 West Africa Ebola virus disease outbreak briefing pack: Foreign Medical Teams International Response. Geneva, World Health Organization, 2014: p. 24. (http://ecampus. msf.org/moodlemsf/pluginfile.php/30615/block_html/content/ WHO\%20Briefing\%20Foreign\%20Medical\%20Teams.pdf, accessed 26 November 2014).

15. Training on hospital management of Ebola Virus Disease (EVD). Manila, World Health Organization Regional Office for the Western Pacific, 2014 (http://www.wpro.who.int/philippines/ mediacentre/features/ebolatraining_materials/en/, accessed 5 January 2015).

16. Guidance on personal protective equipment to be used by healthcare workers during management of patients with Ebola virus disease in U.S. hospitals, including procedures for putting on (donning) and removing (doffing). Atlanta, Centers for Disease Control and Prevention, 2014 (http://www.cdc.gov/vhf/ebola/hcp/ procedures-for-ppe.html, accessed 27 November 2014).

17. WHO updates personal protective equipment guidelines for Ebola response. Geneva, World Health Organization, 2014 (http://www.who.int/mediacentre/news/releases/2014/ebola-ppeguidelines/en/, accessed 27 November 2014).

18. Redman-Maclaren ML et al. Research workshop to research work: initial steps in establishing health research systems on Malaita, Solomon Islands. Health Research Policy and Systems/ BioMed Central, 2010, 8:33. doi:10.1186/1478-4505-8-33 pmid:21034512

19. Guideline on hand hygiene in health care in the context of filovirus disease outbreak response. Geneva, World Health Organization, 2014 (http://apps.who.int/iris/bitstream/10665/ 144578/1/WHO_HIS_SDS_2014.15_eng.pdf?ua=1, accessed 5 December 2014).

20. Devnani $M$ et al. A survey of hand-washing facilities in the outpatient department of a tertiary care teaching hospital in India. Journal of Infection in Developing Countries, 2011, 5:114-118. doi:10.3855/jidc.1003 pmid:21389590

21. Matanock $A$ et al. Ebola virus disease cases among health care workers not working in ebola treatment units - Liberia, JuneAugust, 2014. Morbidity and Mortality Weekly Report, 2014, 63:1077-1081. pmid:25412067

22. Kilmarx PH et al. Ebola virus disease in health care workers Sierra Leone, 2014. Morbidity and Mortality Weekly Report, 2014, 63:1168-1171. pmid:25503921

23. Baron RC, McCormick JB, Zubeir OA. Ebola virus disease in southern Sudan: hospital dissemination and intrafamilial spread. Bulletin of the World Health Organization, 1983, 61:997-1003. pmid:6370486

24. Dowell SF et al. Transmission of Ebola hemorrhagic fever: a study of risk factors in family members, Kikwit, Democratic Republic of the Congo, 1995. Commission de Lutte contre les Epidémies à Kikwit. The Journal of Infectious Diseases, 1999, 179 Suppl 1;S87-91. doi:10.1086/514284 pmid:9988169

25. Review of human-to-human transmission of Ebola virus. Atlanta, Centers for Disease Control and Prevention, 2014 (http://www.cdc. gov/vhf/ebola/transmission/human-transmission.html, accessed 2 November 2014).

26. Jones RM, Brosseau LM. Ebola virus transmission via contact and aerosol - a new paradigm. Center for Infectious Disease Research and Policy, 2014 (http://www.cidrap. umn.edu/news-perspective/2014/11/commentary-ebola- 
virus-transmission-contact-and-aerosol-new-paradigm, accessed 20 November 2014).

27. Brisseau LM, Jones RM. Health workers need optimal respiratory protection for Ebola. Center for Infectious Disease Research and Policy, 2014 (http://www.cidrap.umn.edu/newsperspective/2014/09/commentary-health-workers-need-optimalrespiratory-protection-ebola, accessed 5 November 2014).

28. Chevalier MS et al. Ebola virus disease cluster in the United States - Dallas county, Texas, 2014. Morbidity and Mortality Weekly Report, 2014, 63:1087-1088. pmid:25412069

29. Forrester JD et al.; Centers for Disease Control and Prevention (CDC). Cluster of Ebola cases among Liberian and U.S. health care workers in an Ebola treatment unit and adjacent hospital Liberia, 2014. Morbidity and Mortality Weekly Report, 2014, 63:925-929. pmid:25321070

30. Bannister B, Prygodzicz A, Ippolito G; ETIDE Working Group. Training health care workers to face highly infectious diseases. Clinical Microbiology and Infection, 2009, 15:740-742. doi:10.1111/j.1469-0691.2009.02872.x pmid: 19486076
31. Anonymous. Hospitals prepare plans, drill staff to ensure that potential Ebola patients are identified, isolated, and managed safely. ED Magazine, 2014, 26:138-141.

32. Ashino $Y$ et al. Ebola Virus Disease: Preparedness in Japan. Disaster Medicine and Public Health Preparedness, 2014 1-5. Epub ahead of print. doi:10.1017/dmp.2014.130 pmid:25399765

33. Pesiridis $T$ et al. Development, implementation and evaluation of a disaster training programme for nurses: A Switching Replications randomized controlled trial. Nurse Education in Practice, 2014, pii: S1471-5953(14)00016-X. Epub ahead of print. doi:10.1016/j.nepr.2014.02.001 pmid:24560740

34. Miranda ME, Miranda NLJ. Reston ebolavirus in humans and animals in the Philippines: a review. The Journal of Infectious Diseases, 2011, 204 Suppl 3;5757-760. doi:10.1093/infdis/ jir296 pmid:21987747

35. Pan $Y$ et al. Reston virus in domestic pigs in China. Archives of Virology, 2014, 159:1129-1132. doi:10.1007/s00705-0121477-6 pmid:22996641 


\title{
Intestinal parasites of children and adults in a remote Aboriginal community of the Northern Territory, Australia, 1994-1996
}

\author{
Jennifer Shield, ${ }^{a}$ Kieran Aland, ${ }^{b}$ Thérèse Kearns, ${ }^{c}$ Glenda Gongdjalk, ${ }^{d}$ Deborah Holt, ${ }^{c}$ Bart Currie ${ }^{c e}$ and Paul Prociv ${ }^{f}$ \\ Correspondence to Jennifer Shield (e-mail: J.Shield@latrobe.edu.au).
}

Introduction: Parasitic infections can adversely impact health, nutritional status and educational attainment. This study investigated hookworm and other intestinal parasites in an Aboriginal community in Australia from 1994 to 1996.

Methods: Seven surveys for intestinal parasites were conducted by a quantitative formol-ether method on faecal samples. Serological testing was conducted for Strongyloides stercoralis and Toxocara canis IgG by enzyme-linked immunosorbent assays.

Results: Of the 314 participants, infections were as follows: Trichuris trichiura (86\%); hookworm, predominantly Ancylostoma duodenale (36\%); Entamoeba spp. (E. histolytica complex [E. histolytica, E. dispar and E. moskovski], E. coli and E. hartmanni) (25\%); S. stercoralis (19\%); Rodentolepis nana (16\%); and Giardia duodenalis (10\%). Serological diagnosis for 29 individuals showed that $28 \%$ were positive for S. stercoralis and $21 \%$ for T. canis. There was a decrease in the proportion positive for hookworm over the two-year period but not for the other parasite species. The presence of hookworm, T. trichiura and Entamoeba spp. was significantly greater in 5-14 year olds $(n=87)$ than in 0-4 year olds $(n=41)$, while the presence of S. stercoralis, $R$. nana, G. duodenalis and Entamoeba spp. in 5-14 year olds was significantly greater than $15-69$ year olds $(n=91)$.

Discussion: Faecal testing indicated a very high prevalence of intestinal parasites, especially in schoolchildren. The decrease in percentage positive for hookworm over the two years was likely due to the albendazole deworming programme, and recent evidence indicates that the prevalence of hookworm is now low. However there was no sustained decrease in percentage positive for the other parasite species.

$\mathrm{P}$ arasitic infections adversely affect health. Heavy infections with helminths cause clinical disease including anaemia, diarrhoea and malabsorption of nutrients. ${ }^{1-5}$ Moderate or light infections cause morbidity by adversely affecting nutritional status ${ }^{6}$ and affect educational outcomes by impairing cognitive processes. ${ }^{7}$ Trichuris trichiura infection has been associated with poor immunogenicity from BCG vaccination. ${ }^{8}$

Several intestinal parasite species occur in Australia with a high prevalence in rural and remote Aboriginal communities. Hookworm (predominantly Ancylostoma duodenale) occurs mainly north of $22^{\circ} \mathrm{S}$ particularly near the coast. ${ }^{9-12}$ Whipworm (T. trichiura) is rare in Western Australia, ${ }^{9}$ more common in Queensland $^{1}$ and also occurs in the Northern Territory (NT). ${ }^{10,13,14}$ Strongyloides stercoralis is more widespread, occurring throughout the northern two thirds of Australia (Jennifer Shield, La Trobe University, unpublished collated data). Dwarf tapeworm (Rodentolepis nana [= Hymenolepis nana]) and the protozoan Giardia duodenalis are common in Aboriginal communities throughout Western Australia, ${ }^{9}$ Queensland ${ }^{1}$ and the NT. ${ }^{10,13}$ Entamoeba spp. occur throughout Australia. ${ }^{1,15,16}$ Among the pathogenic species of intestinal protozoa, Entamoeba histolytica is the second leading cause of death globally after malaria, ${ }^{15}$ and G. duodenalis causes chronic diarrhoea, malabsorption and weight loss and contributes to stunting and cognitive impairment in children. ${ }^{17}$

In the past, morbidity of parasitic infections was assessed on a species-by-species basis. Current evidence, however, suggests that when a person is

\footnotetext{
La Trobe University, Bendigo, Victoria, Australia.

Queensland Museum, Brisbane, Queensland, Australia

Menzies School of Health Research, Darwin, Northern Territory, Australia.

Ngalkanbuy Health Centre, Galiwin'ku, Northern Territory, Australia

Royal Darwin Hospital, Darwin, Northern Territory, Australia.

University of Queensland, Brisbane, Queensland, Australia.

Submitted: 14 January 2015; Published: 6 March 2015

doi: 10.5365/wpsar.2015.6.1.008
} 
infected with two or more parasite species, there is an additive or multiplicative impact on nutrition and organ pathology. ${ }^{18}$

There is a shortage of published surveys of intestinal parasites in Australia. This paper documents the prevalence of intestinal parasites in a remote Aboriginal community in Australia from 1994 to 1996. Historical data such as these are rare and are of intrinsic value. In addition, they provided baseline data with which to compare the current situation, enabling the evaluation of deworming strategies.

\section{METHODS}

\section{Subjects and location}

This study was conducted in a remote township, in north-eastern Arnhem Land between July 1994 and October 1996. Most residents belong to the Yolngu group of Aboriginal Australians.

\section{Collection of faecal samples}

Each household was visited by the parasitologist and health educator. Information about hookworm infection, transmission and diagnosis was given in the local language using prepared illustrations, and then a faecal sample was requested. Each person was given a labelled rectangular plastic container with a snap-on lid and a brown paper bag. The containers were collected the following morning. Sampling occurred during seven time periods: July 1994 (1 to 18 July), October 1994 (30 September to 19 October), December 1994 (8 to 19 December), May 1995 (5 to 22 May), August 1995 (4 to 19 August), December 1995 (20 November to 19 December) and October 1996 (exact dates not recorded). Collections in November and December were during the wet season; the other collections were during the dry season.

\section{Examination of faecal samples}

In the field laboratory, a direct saline wet preparation of the faecal specimen was examined microscopically as soon as possible, and a $0.91 \mathrm{~mL}$ aliquot of faeces was preserved in $5-10 \mathrm{~mL} 4 \%$ formaldehyde for later examination by a quantitative formol-ether count method. Hookworm and T. trichiura eggs were counted and the presence of $S$. stercoralis larvae, $R$. nana eggs, G. duodenalis, Entamoeba spp. and other protozoa cysts or trophozoites were noted. The number of eggs per $\mathrm{mL}$ faeces was calculated by multiplying the count by a factor (total volume of faecal suspension/[volume of the faecal sample $x$ volume of sample counted]). The size of the multiplication factor varied between 10 and 42 . The counts were adjusted for faecal consistency using the Stoll correction factors. ${ }^{19}$

\section{Determination of hookworm species}

Vermiculite cultures of 10 faecal samples with a substantial number of eggs in the direct smear were prepared to determine the species of hookworm based on the morphology of the cultured filariform larvae. ${ }^{20}$

Adult hookworms were collected from the digestive system on autopsy of stray dogs. Twelve adult worms were measured and examined in detail to determine the species.

\section{Serology (specific serum immunoglobulin G [IgG] tests)}

Venous blood was collected from 39 adults and children as part of their normal routine testing and IgG levels against Strongyloides and Toxocara were measured using enzyme-linked immunosorbent assay. The Strongyloides assay used S. ratti $\mathrm{L} 3$ as the antigen and had an estimated sensitivity of $93 \%$ and specificity of $95 \%{ }^{21}$ The Toxocara assay used $T$. canis excretory/secretory antigen as the antigen and had an estimated sensitivity of $91 \%$ and specificity of $86 \% .^{22}$

\section{Statistical analysis}

A parasitic infection was defined as specimens positive by the quantitative formol-ether method only. Survey results included participants who were present at more than one survey; only the first examination was included. Geometric means of egg counts of infected people were calculated using logarithm to base e (In) transformed data and egg counts recovered by antilogarithm. When the egg counts for $A$. duodenale and $T$. trichiura were grouped using a logarithmic scale, the plotted frequencies had a normal distribution, indicating that statistical methods applicable to normal distributions were valid when performed on logarithmically transformed egg count 
data. Intensity of infection was classified according to World Health Organization categories ${ }^{23}$ (assuming that eggs $/ \mathrm{mL}$ is approximately equivalent to eggs/g faeces).

The difference of two independent proportions was compared by calculation of the $\mathrm{Z}$ ratio, taking the two-tail value for $P$ (probability). Where the number infected or the number negative was too small for this test, the Fisher's Exact Test was used. Ln transformed mean egg counts were compared using a two-tailed $t$-test for independent samples and unequal variances. Frequency data were analysed using Yates' chi-square test. A $P$-value of $<0.05$ was considered significant. All statistical calculations were performed by the online calculators provided by the VassarStats Web Site for Statistical Calculations (http://www.vassarstats. net/). Graphs were drawn using Excel.

\section{Ethics statement}

Ethical approval for this study was obtained from the Human Research Ethics Committee of the NT Department of Health and Menzies School of Health Research (Registration No. EC00153), approval number 94/19. Everyone who tested positive for parasites by the field smear was notified and asked to go to the health centre for treatment.

\section{RESULTS}

\section{Intestinal parasites: combined faecal count data from all surveys}

Of the 314 participants, the overall percentage positive for intestinal parasites was $89 \%$ and for one or more helminth species was $88 \%$. The most common parasite species was T. trichiura $(86 \%$ positive) followed by hookworm (36\%), Entamoeba spp. [E. histolytica complex: E. coli and E. hartmanni] (29\%); S. stercoralis (19\%), R. nana (16\%) and G. duodenalis (10\%). Enterobius vermicularis, Chilomastix mesnilii, Retortamonas intestinalis and Blastocystis spp. were present in a small number of participants.

The intensity of infection for the 314 participants for hookworm was: negative: $64 \%$; light (1-1999 eggs $/ \mathrm{mL}$ faeces): 35\%; moderate (2000-3999 eggs $/ \mathrm{mL})$ : $1 \%$; and for T. trichiura was: negative: $16 \%$; light (1-999 eggs $/ \mathrm{mL}): \quad 50 \%$; moderate (1000-9999 eggs $/ \mathrm{mL}$ ): $30 \%$; and heavy ( $\geq 10000$ eggs $/ \mathrm{mL}$ ): $4 \%$.
Serological diagnosis for 39 individuals showed that $28 \%$ were positive and $18 \%$ equivocal for S. stercoralis, and $21 \%$ were positive and $5 \%$ equivocal for $T$. canis. The median age for the 21 persons for whom ages were known was 32 years (IQR 24 to 41).

\section{Comparison of the seven surveys}

There were 55 (18\%) participants represented in more than one survey. There was significant variation in the percentage of participants positive for hookworm and S. stercoralis over the study period (Table 1). The percentage positive for hookworm was high in October 1994 preceding the wet season, low in August 1995 following the introduction of an albendazole treatment programme and high in December 1995 during the wet season. There was a significant decrease between October 1994 and October $1996(P<0.0002$; Table 1). The percentage positive for $S$. stercoralis was high in May 1995, low in August 1995 following the introduction of albendazole, high in December 1995 (wet season) and low in October 1996 (Table 1). There was no significant variation in the percentage of participants positive for $T$. trichiura, $R$. nana, G. duodenalis or Entamoeba spp.

There was no significant variation in the geometric mean egg counts of positive samples during the seven surveys for hookworm (Table 1). For T. trichiura, the geometric mean of egg counts in December 1995 (2200 eggs $/ \mathrm{mL}$ ) was significantly greater than those of all the other surveys $(P<0.001)$ (Table 1$)$. This result was likely due to markedly increased egg counts in children (0-14 years) at that time.

\section{Comparisons of age groups and sex}

The median age of the 219 participants whose age was known was 11 years (IQR 6 to 29) (Figure 1); 129 children were aged 0-14 years (approximately $37 \%$ of the child population) and 91 adults were aged 15 years and above (approximately $12 \%$ of the adult population). There was a significantly greater percentage positive in schoolchildren (5-14 years) compared with preschool children (0-4 years) for any parasitic infection, hookworm, T. trichiura and Entamoeba spp, and a significantly lower percentage positive in adults $(\geq 15$ years) compared with schoolchildren for S. stercoralis, R. nana, G. duodenalis and Entamoeba spp (Figure 1). Hookworm, T. trichiura, R. nana and 
Table 1. Number and proportion of participants who tested positive for intestinal parasites by survey, Northern Territory, Australia, July 1994 to October 1996, $n=383$

\begin{tabular}{|c|c|c|c|c|c|c|c|c|c|c|}
\hline \multirow[b]{2}{*}{$\begin{array}{l}\text { Survey } \\
\text { date }\end{array}$} & \multirow{2}{*}{$\begin{array}{l}\text { No. } \\
\text { people } \\
\text { tested }\end{array}$} & \multirow{2}{*}{$\begin{array}{l}\text { Any parasite } \\
\text { Positive } \\
\text { No. (\%) }\end{array}$} & \multicolumn{2}{|c|}{ Hookworm } & \multicolumn{2}{|c|}{ T. trichiura } & \multirow{2}{*}{$\begin{array}{c}\text { S. stercoralis } \\
\text { Positive } \\
\text { No. (\%) }\end{array}$} & \multirow{2}{*}{$\begin{array}{c}\text { R. nana } \\
\text { Positive } \\
\text { No. (\%) }\end{array}$} & \multicolumn{2}{|c|}{ G. duodenalis Entamoeba spp } \\
\hline & & & $\begin{array}{c}\text { Positive } \\
\text { No. (\%) }\end{array}$ & $\begin{array}{c}\text { Geom mean } \\
(\operatorname{eggs} / \mathrm{mL}) \\
{[\operatorname{Exp}(\mathrm{Cl})]}\end{array}$ & $\begin{array}{l}\text { Positive } \\
\text { No. (\%) }\end{array}$ & $\begin{array}{c}\text { Geom mean } \\
(\operatorname{eggs} / \mathrm{mL}) \\
{[\operatorname{Exp}(\mathrm{Cl})]}\end{array}$ & & & $\begin{array}{l}\text { Positive } \\
\text { No. (\%) }\end{array}$ & $\begin{array}{l}\text { Positive } \\
\text { No. (\%) }\end{array}$ \\
\hline \multirow[t]{2}{*}{ Jul 1994} & 62 & 57 (92) & $19(31)$ & 200 & $53(86)$ & 820 & $13(21)$ & $15(24)$ & $10(16)$ & $18(29)$ \\
\hline & & & & [120-350] & & [520-1300] & & & & \\
\hline \multirow[t]{2}{*}{ Oct 1994} & 76 & 73 (94) & $43(57)^{*}$ & 180 & 68 (89) & 560 & $13(16)$ & $13(16)$ & $9(11)$ & $22(27)$ \\
\hline & & & & [120-250] & & [390-800] & & & & \\
\hline \multirow[t]{2}{*}{ Dec 1994} & 37 & $31(84)$ & $17(46)$ & 110 & $29(78)$ & 400 & $4(11)$ & $3(8)$ & $3(8)$ & $11(30)$ \\
\hline & & & & [61-190] & & [260-620] & & & & \\
\hline \multirow[t]{2}{*}{ May 1995} & 48 & $44(92)$ & $16(33)$ & 120 & $40(83)$ & 800 & $17(35)^{*}$ & 7 (15) & $0(0)$ & $10(21)$ \\
\hline & & & & [73-200] & & [460-1400] & & & & \\
\hline Jun 1995 & & Single-dose alk & pendazole o & eworming prog & yramme be & gins, replacing & previous progr & mmes usir & ng pyrantel an & bendazole. \\
\hline \multirow[t]{2}{*}{ Aug 1995} & 63 & $57(90)$ & $7(11)^{* \dagger}$ & 130 & $53(84)$ & 520 & $6(19)^{*}$ & $12(19)$ & $4(6)$ & $23(37)$ \\
\hline & & & & [42-420] & & [360-740] & & & & \\
\hline \multirow[t]{2}{*}{ Dec 1995} & 44 & $41(93)$ & $15(34)^{*}$ & 140 & $38(86)$ & $2200^{* \ddagger}$ & $13(30)^{*}$ & $12(27)$ & $5(11)$ & $17(27)$ \\
\hline & & & & [79-250] & & [1400-3300] & & & & \\
\hline \multirow[t]{2}{*}{ Oct 1996} & 53 & $46(87)$ & $13(25)^{\dagger}$ & 100 & $44(83)$ & 380 & $4(8)^{*}$ & 7 (13) & $5(9)$ & $21(40)$ \\
\hline & & & & [53-180] & & [230-640] & & & & \\
\hline \multirow[t]{2}{*}{ Total $^{\S}$} & 383 & 349 (91) & $130(34)$ & 100 & 325 (85) & 650 & 70 (18) & 69 (18) & 36 (9) & $122(32)$ \\
\hline & & & & [110-190] & & [550-780] & & & & \\
\hline
\end{tabular}

Exp (CI), exponentially recovered confidence interval; Geom mean, geometric mean for those infected.

* Significant difference with the previous survey.

$\dagger$ Significant difference with the survey conducted approximately one year earlier.

¥ Significant difference with the geometric means of all other surveys.

$\S$ Some participants were included in more than one survey.

Figure 1. Proportion of intestinal parasites detected among participants by age group* and species, Northern Territory, Australia, July 1994 to October 1996

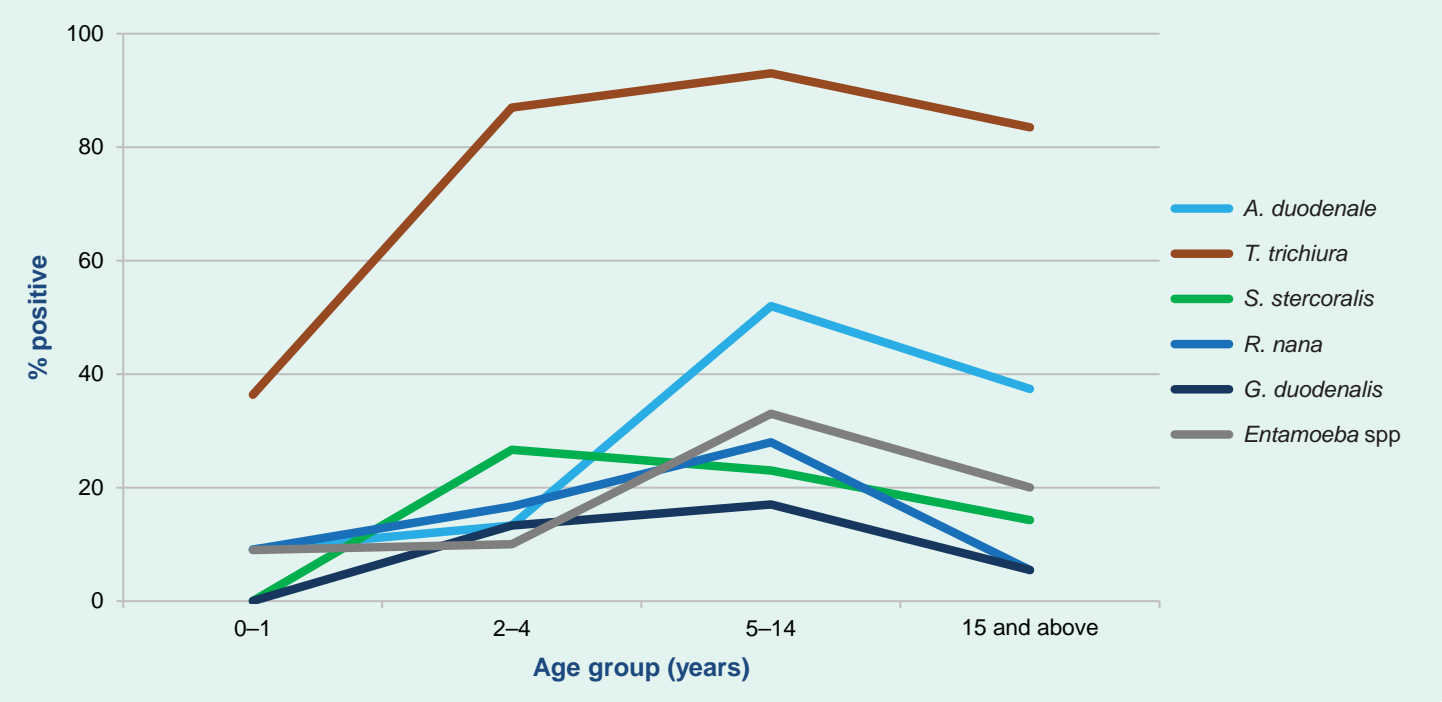

* Age group was available for 219 (70\%) of participants, 0-4 years age group was divided into 0-1 and 2-4. 
Entamoeba spp. were present in 0-1 year old children. All six species were present in 2-4 year old children (Figure 1).

For T. trichiura, the geometric mean egg count in infected schoolchildren (1200 eggs $/ \mathrm{mL}$ ) was significantly greater than in both preschool children (400 eggs $/ \mathrm{mL})$ and adults $(410$ eggs $/ \mathrm{mL})(P<0.001)$. For hookworm, there was no significant difference between geometric mean egg counts for the age groups.

The sex was known for 100 males and 115 females. The percentage positive in males was significantly greater than in females for hookworm (47\% compared with $30 \% ; P=0.012)$, T. trichiura (92\% compared with $79 \% ; P=0.0082$ ) and $G$. duodenalis ( $17 \%$ compared with $6 \% ; P=0.011$ ).

\section{Polyparasitism}

Of the 314 participants, 152 (48\%) were infected with more than one helminth species and 109 (35\%) were infected with one or more helminth species and one or more protozoa species (Figure 2). There was a statistically significant association of helminth infection with protozoa infection $(P=0.0039)$.

\section{Hookworm species}

Infective larvae recovered from vermiculite faecal cultures were all identified as Ancylostoma spp., most likely Ancylostoma duodenale. Adult Ancylostoma collected from dogs were all identified as $A$. caninum.

\section{DISCUSSION}

These results indicate a very high prevalence of infection with one or more intestinal parasite species in this community between 1994 and 1996 despite the deworming programme in children that was conducted from before the study began until the present time. In 1994, the standard treatment was pyrantel $20 \mathrm{mg} / \mathrm{kg}$ as a single dose or mebendazole $100 \mathrm{mg}$ twice daily for three days. From May 1995 albendazole (single dose of $200 \mathrm{mg}$ for children 8 to $<10 \mathrm{~kg}$ body weight and $400 \mathrm{mg}$ for children $\geq 10 \mathrm{~kg}$ ) was the usual anthelmintic treatment during yearly child health checks; for adults and children with suspected or confirmed gastrointestinal helminths, the standard treatment was daily albendazole $400 \mathrm{mg}$ for three days. The parasite species detected by

\section{Figure 2. Proportion of helminth infections with and without protozoa infection, Northern Territory, Australia, July 1994 to October 1996, $(n=314)^{*}$}

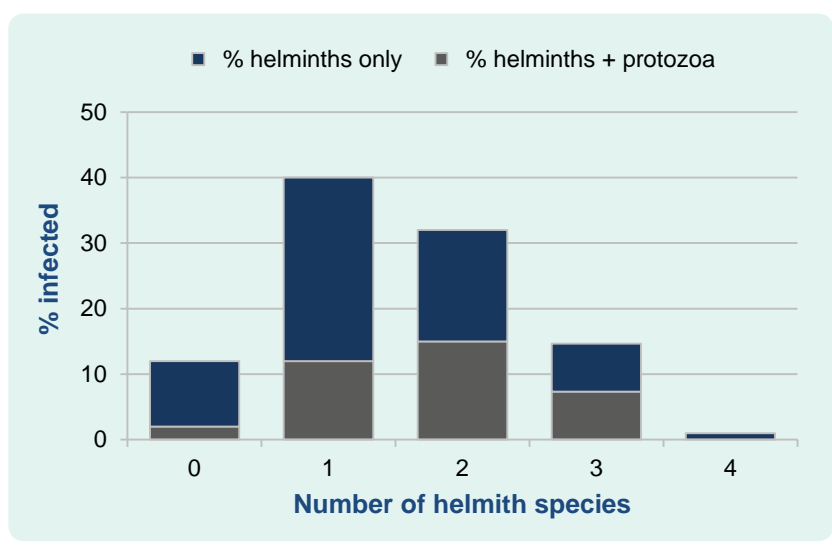

* There was a statistically significant association of helminth infection with protozoa infection $(P=0.0039)$.

faecal testing were the same as in two smaller studies in the same region at about the same time, ${ }^{10,13}$ but the percentages positive in our study were greater. This likely reflects the greater sensitivity of the quantitative formolether method. Even though this method was much more sensitive than direct smears due to the relatively large amount of faeces examined, it likely underestimated the true prevalence; for example, $28 \%$ of a small group ( $n=39$ ) were positive for $S$. stercoralis by serology compared with $19 \%$ positive by formol-ether count $(n=314)$. A study of a similar method using ethyl acetate instead of ether showed that it was much less sensitive than agar plate culture in detecting S. stercoralis. ${ }^{24}$ A positive test by serology indicates a current infection because if the $S$. stercoralis are eliminated, the antibody levels decrease to negative within three to six months of effective treatment. ${ }^{25,26}$

Although the larvae harvested from the vermiculite cultures were Ancylostoma sp, probably A. duodenale, the presence of Necator americanus cannot be excluded. A. ceylanicum may also be present, as it is present in dogs in Australia ${ }^{27}$ and has also been detected in people. ${ }^{28}$ The observed seasonal variations in the percentage positive for hookworm provide additional evidence that the hookworm was at least predominantly $A$. duodenale. The variation is likely due to larval hypobiosis during the dry season and increased transmission during the wet season. ${ }^{29}$ The lifespan of adult $A$. duodenale is approximately six months. Larvae that enter the body late in the wet season do not develop but remain in the tissues and mature late in the dry season. Treatment 
with pyrantel eradicates adult $A$. duodenale but not the dormant larvae. ${ }^{29}$ The peak in percentage positive in October 1994, late in the dry season, is consistent with this. Increased transmission during the wet season accounts for the high percentage positive for A. duodenale in December 1994 and 1995.

The very high prevalence of infection with two or more intestinal parasites observed in this study is of concern; individuals with multiple infections suffer increased morbidity ${ }^{18}$ due to the differing pathologies caused by the different parasitic species. A greater prevalence of hookworm, T. trichiura and G. duodenalis in males than females has been observed elsewhere. ${ }^{30}$

The greater percentages positive for intestinal parasites in school-aged children compared with other age groups follows patterns elsewhere. ${ }^{18}$ There was an appreciable percentage positive in very young children of 2-4 years at this critical stage in their development. The finding that $R$. nana and $G$. duodenalis infections occur primarily in children under 15 years of age is consistent with findings in Aboriginal communities throughout Western Australia. ${ }^{9}$ The percentage of participants positive for hookworm and T. trichiura in schoolchildren compared with adults were not significantly different, but the infection intensity of T. trichiura in schoolchildren was significantly greater than in adults.

The finding of an appreciable percentage of adult participants positive for hookworm, T. trichiura and S. stercoralis justifies regular anthelmintic treatment of adults as well as children. The lower percentage of adult participants positive for S. stercoralis compared with schoolchildren is of interest since infection with this species can be life-long in the absence of effective treatment. $^{31}$ Single-dose albendazole does not usually eliminate the infection. ${ }^{25}$

The presence of $A$. caninum in dogs raises the possibility of zoonotic infections in humans, which may be asymptomatic or cause eosinophilic enteritis. ${ }^{32}$ Seroepidemiological studies showed it to be common in Queensland in the 1990s. ${ }^{32}$ While eosinophilic enteritis has been recognized in the non-Aboriginal population of the NT, it has not been documented as a clinical issue in the Aboriginal population, ${ }^{33}$ possibly because of the albendazole deworming programme.
T. canis is common in dogs and is found as eggproducing adult worms mainly in pups. Our finding of specific IgG against $T$. canis in $21 \%$ of people tested indicates a significant level of human exposure to infection (putatively via egg ingestion). By way of comparison, $43 \%$ of another Aboriginal group in far north-eastern Arnhem Land, ${ }^{10} 11.1 \%$ of another NT group and $5.7 \%$ of the general Australian population ${ }^{34}$ were seropositive in the 1990s. The clinical significance of $T$. canis in the Aboriginal population is unknown. The larvae can cause hepatic disease (visceral larva migrans) or eye lesions (ocular larva migrans). ${ }^{19}$ Visceral larva migrans has been occasionally diagnosed in the non-Aboriginal population of the NT, but confirmed cases in the Aboriginal population have not occurred in recent years (Bart Currie, Royal Darwin Hospital, unpublished data).

The variations in S. stercoralis percentage positive are difficult to interpret. Intermittent larval shedding has been documented in this species $^{35}$ and may explain the variations seen. Another possibility is that pyrantel, mebendazole or albendazole treatment was not eradicating infection but causing only a temporary decrease in larval output. Currently, the use of ivermectin is greatly improving the efficacy of treatment. ${ }^{25}$ The effectiveness of community-wide ivermectin treatment to reduce $S$. stercoralis infection is currently being assessed. ${ }^{26}$

The results of the October 1996 survey suggest that the albendazole treatment programme was reducing the prevalence of hookworm as the percentage of participants positive for hookworm decreased significantly at a time of year when an increase would be expected from maturation of dormant larvae of $A$. duodenale. This result is also consistent with the eradication of the dormant larvae in those who had received albendazole treatment. Initial treatment with albendazole was likely responsible for the drop in percentage positive for hookworm in August 1995. Recent work indicates that $A$. duodenale is now well controlled, ${ }^{36,37}$ but a high proportion of the children tested were positive for $T$. trichiura and R. nana.${ }^{37}$ A recent review of laboratory data indicated that the detection rates of $T$. trichiura in the NT have substantially decreased over the last decade. ${ }^{14}$ Singledose albendazole is much less effective at eliminating T. trichiura in children than in adults ${ }^{38}$ and is not effective against $R$. nana. ${ }^{39}$ Our results are consistent 
with this. The efficacy of albendazole against $T$. trichiura, $R$. nana and G. duodenalis is improved if given daily for three days. ${ }^{38,39}$

It is not possible to conclude whether the Entamoeba spp. observed were of public health significance. Determination of the extent of the recognized pathogens E. histolytica and E. moshkovski present in participants would require molecular faecal testing that is available now but not at the time of the study.

A factor that probably contributes to the high prevalence of parasites is the large number of people per house, ${ }^{40}$ putting pressure on food preparation, washing and sanitary facilities. ${ }^{41}$ Recently, there has been an attempt to address this issue with the addition of new and renovated houses.

There is an urgent need for a more holistic approach to the control of the remaining parasites, combining health promotion and adequate treatment. A key ingredient is community empowerment through respectful adult community education.

In this study, the samples were not randomly selected, sample sizes were small and largely made up of newly recruited participants. There was variation in the proportion of children (45-70\%) and in the maleto-female ratio (0.8 to 1.7 ) from survey to survey. The pooled values used to compare different age groups, sex and multiple infections did not take into account the seasonal variation in hookworm or S. stercoralis. Documentation of anthelmintic treatment at the individual level depended on health centre records which were incomplete; therefore we examined the effect of the treatment programme on the prevalence of the parasites. There was no attempt to distinguish between new infections and those detected in the previous surveys.

\section{CONCLUSIONS}

In spite of the above limitations, this study highlights the very high prevalence of infection with intestinal parasites, frequently with more than one species, in this remote Aboriginal community in the 1990s, particularly in schoolchildren. The survey data showed that, following the introduction of a yearly single-dose albendazole treatment programme for children, there was a significant reduction in the percentage of participants positive for hookworm but no significant change in the other species of intestinal parasites. This historical data adds to our limited knowledge of parasitic infections in the 1990s and provides a base line for comparison with the current situation, enabling the evaluation of deworming strategies.

\section{Conflicts of interest}

None declared.

\section{Funding}

This work was funded by a National Health and Medical Research Council grant through the Public Health Research and Development Committee to the Ngalkanbuy Health Centre at Galiwin'ku for hookworm research. Kieran Aland was supported by a Commonwealth Postgraduate Scholarship at the University of Queensland.

\section{Acknowledgements}

The authors gratefully acknowledge the people of the Galiwin'ku community who provided faecal and blood samples to enable this study to take place; John Walker and Rogan Lee, Pathology West, Institute of Clinical Pathology and Medical Research, Westmead, Sydney, NSW, who provided the serology results and details of the method used, respectively; and David Shield, who was the statistical consultant for the data analysis.

\section{References:}

1. Jose DG, Welch JS. Growth retardation, anaemia and infection, with malabsorption and infestation of the bowel. The syndrome of protein-calorie malnutrition in Australian Aboriginal children. The Medical Journal of Australia, 1970, 1:349-356. pmid:5439141

2. Layrisse $\mathbf{M}$, Roche $\mathbf{M}$. The relationship between anemia and hookworm infection. Results of surveys of rural Venezuelan population. American Journal of Hygiene, 1964, 79:279-301. pmid: 14159948

3. Lucas SB et al. Aberrant form of Hymenolepis nana: possible opportunistic infection in immunosuppressed patients. Lancet, 1979, 2:1372-1373. doi:10.1016/S0140-6736(79)92859-9 pmid:92715

4. Cooper ES, Bundy DA, Henry FJ. Chronic dysentery, stunting, and whipworm infestation. Lancet, 1986, 2:280-281. doi:10.1016/ S0140-6736(86)92093-3 pmid:2874297

5. Speare R. Fatal strongyloidiasis: lessons from the literature. In: Second National Workshop on Strongyloidiasis. Brisbane, Queensland, 2003 (http://www.jcu.edu.au/school/phtm/PHTM/ss/ acrrm-cd/CD-Index.pdf, accessed 11 February 2015).

6. Stephenson LS et al. Treatment with a single dose of albendazole improves growth of Kenyan schoolchildren with hookworm, Trichuris trichiura, and Ascaris lumbricoides infections. The American Journal of Tropical Medicine and Hygiene, 1989, 41:78-87. pmid:2764230 
7. Ezeamama AE et al. Helminth infection and cognitive impairment among Filipino children. The American Journal of Tropical Medicine and Hygiene, 2005, 72:540-548. pmid:15891127

8. Elias $D$ et al. Poor immunogenicity of BCG in helminth infected population is associated with increased in vitro TGF-beta production. Vaccine, 2008, 26:3897-3902. doi:10.1016/j. vaccine.2008.04.083 pmid:18554755

9. Jones HI. Intestinal parasite infections in Western Australian Aborigines. The Medical Journal of Australia, 1980, 2:375-380. pmid:7453610

10. Flannery G, White N. Immunological parameters in northeast Arnhem Land Aborigines: consequences of changing settlement patterns and lifestyles. In: Schell M, Bilsborough A, editors. Urban Ecology and Health in the Third World. Society for the Study of Human Biology Symposium Series. Melbourne, Cambridge University Press, 1993, pp. 202-220.

11. Prociv P, Luke RA. The changing epidemiology of human hookworm infection in Australia. The Medical Journal of Australia, 1995, 162:150-154. pmid:7854229

12. Hopkins RM et al. The prevalence of hookworm infection, iron deficiency and anaemia in an aboriginal community in north-west Australia. The Medical Journal of Australia, 1997, 166:241244. pmid: 9076267

13. Fryar D, Hagan S. Pilot screening program for intestinal parasites and anaemia in adults in a Top End Aboriginal community. Northern Territory Communicable Diseases Bulletin, 1997, $4: 20-21$.

14. Crowe AL et al. Decreasing prevalence of Trichuris trichiura (whipworm) in the Northern Territory from 2002 to 2012. The Medical Journal of Australia, 2014, 200:286-289. doi:10.5694/mja13.00141 pmid:24641155

15. van $\mathrm{Hal} \mathrm{SJ}$ et al. Amoebiasis: current status in Australia. The Medical Journal of Australia, 2007, 186:412-416. pmid: 17437396

16. McCarthy JS et al. Endemic invasive amoebiasis in northern Australia. The Medical Journal of Australia, 2002, 177:570. pmid: 12429010

17. Ali SA, Hill DR. Giardia intestinalis. Current Opinion in Infectious Diseases, 2003, 16:453-460. doi:10.1097/00001432200310000-00012 pmid:14501998

18. Drake LJ, Bundy DA. Multiple helminth infections in children: impact and control. Parasitology, 2001, 122 Suppl;S73-81. doi:10.1017/S0031182000017662 pmid:11442199

19. Beaver PC, Jung RC, Cupp EW. Clinical Parasitology. 9th ed. Philadelphia, Lea \& Febiger, 1984.

20. Basic laboratory methods in medical parasitology. Geneva, World Health Organization, 1991.

21. Grove DI. Diagnosis. In: Grove DI, editor. Strongyloidiasis a major roundworm infection of man. London, Taylor and Francis, 1989, pp. 175-197.

22. Jacquier $P$ et al. Immunodiagnosis of toxocarosis in humans: evaluation of a new enzyme-linked immunosorbent assay kit. Journal of Clinical Microbiology, 1991, 29:1831-1835. pmid: 1774303

23. Montresor A et al. Guidelines for the evaluation of soil-transmitted helminthiasis and schistosomiasis at community level: a guide for managers of control programmes. Geneva, World Health Organization, 1998.

24. Intapan PM et al. Comparison of the quantitative formalin ethyl acetate concentration technique and agar plate culture for diagnosis of human strongyloidiasis. Journal of Clinical Microbiology, 2005, 43:1932-1933. doi:10.1128/ JCM.43.4.1932-1933.2005 pmid:15815023
25. Page WA, Dempsey K, McCarthy JS. Utility of serological follow-up of chronic strongyloidiasis after anthelminthic chemotherapy. Transactions of the Royal Society of Tropical Medicine and Hygiene, 2006, 100:1056-1062. doi:10.1016/j. trstmh.2005.12.006 pmid:16551471

26. Kearns $T$ et al. Ivermectin mass drug administration program to treat endemic scabies and strongyloidiasis in a remote aboriginal community in northern Australia. Tropical Medicine \& International Health, 2011, 16 S1;198-199.

27. Palmer CS et al. The veterinary and public health significance of hookworm in dogs and cats in Australia and the status of A. ceylanicum. Veterinary Parasitology, 2007, 145:304-313. doi:10.1016/j.vetpar.2006.12.018 pmid:17276602

28. Koehler AV et al. Genetic characterization of selected gastrointestinal parasites associated with humans using a mutation scanningcoupled approach. Electrophoresis, 2013, 34:1720-1722. doi:10.1002/elps.201300100 pmid:23592267

29. Prociv P, Luke RA. Evidence for larval hypobiosis in Australian strains of Ancylostoma duodenale. Transactions of the Royal Society of Tropical Medicine and Hygiene, 1995, 89:379. doi:10.1016/0035-9203(95)90016-0 pmid:7570868

30. Zuk M, McKean KA. Sex differences in parasite infections: patterns and processes. International Journal for Parasitology, 1996, 26:1009-1023. doi:10.1016/S0020-7519(96)00086-0 pmid:8982783

31. Adams M, Page W, Speare R. Strongyloidiasis: an issue in Aboriginal communities. Rural and Remote Health, 2003, 3:152. pmid: 15877491

32. Prociv P. Zoonotic hookworm infections (ancylostomosis). In Palmer SR, Lord Soulsby, Simpson DIH, editors. Zoonoses: biology, clinical practice, and public health control. Oxford, Oxford University Press, 1998, p. 803-822.

33. Currie B, Anstey N. Eosinophilic enteritis in the Northern Territory. The Medical Journal of Australia, 1991, 154:71. pmid: 1984596

34. Conner G. Diagnosis and epidemiology of Toxocara canis infection in humans (Masters of Applied Science thesis). Sydney, University of Technology, 1990

35. Dreyer $\mathrm{G}$ et al. Patterns of detection of Strongyloides stercoralis in stool specimens: implications for diagnosis and clinical trials. Journal of Clinical Microbiology, 1996, 34:2569-2571. pmid:8880521

36. Davies J et al. Hookworm in the Northern Territory: down but not out. The Medical Journal of Australia, 2013, 198:278-281. doi:10.5694/mja12.11615 pmid:23496406

37. Kearns $T$ et al. Faecal parasitology of human specimens collected from a remote Aboriginal community in the Northern Territory. ASM/ACTM Poster abstract, Parasitology Masterclass 14-17 July, 2011, Cairns. Annals of the ACTM, 2011, 12:55.

38. Horton J. Albendazole: a review of anthelmintic efficacy and safety in humans. Parasitology, 2000, 121 Suppl:S113-132. doi:10.1017/S0031182000007290 pmid:11386684

39. Reynoldson JA et al. Efficacy of albendazole against Giardia and hookworm in a remote Aboriginal community in the north of Western Australia. Acta Tropica, 1998, 71:27-44. doi:10.1016/ S0001-706X(98)00048-5 pmid:9776141

40. Castles I. Census counts for small areas: Northern Territory 1991 Census of Population and Housing. Canberra, Australian Bureau of Statistics, 1993.

41. Li SQ et al. From infancy to young adulthood: health status in the Northern Territory. Darwin, Northern Territory Government Department of Health and Community Services, 2006. 


\title{
Brief behavioural surveys in routine HIV sentinel surveillance: a new tool for monitoring the HIV epidemic in Viet Nam
}

\author{
Duong Cong Thanh, ${ }^{a}$ Nguyen Tran Hien, ${ }^{a}$ Nguyen Anh Tuan, ${ }^{a}$ Hoang Thi Thanh Ha, ${ }^{a}$ Pham Hong Thang, \\ Nguyen Thi Thanh Ha, ${ }^{a}$ Le Anh Tuan, ${ }^{a}$ Tran Dai Quang, ${ }^{a}$ Tran Hong Tram, ${ }^{a}$ Nguyen Le Hai, ${ }^{\circ}$ \\ Phan Thi Thu Huong, ${ }^{b}$ Vo Hai Son, ${ }^{b}$ Bui Hoang Duc, ${ }^{b}$ Nguyen Thien Nga, ${ }^{c}$ David Jackac and Keith Sabin ${ }^{c}$ \\ Correspondence to Duong Cong Thanh (e-mail: congthanhnihe@yahoo.com).
}

In this report we describe a new approach in HIV sentinel surveillance that was piloted in Viet Nam in 2009 and is currently being rolled out in all provinces. It comprises a brief behavioural questionnaire added to the HIV sentinel surveillance surveys conducted routinely among people who inject drugs, female sex workers and men who have sex with men. Timely reporting of data from this system has resulted in improvements to HIV prevention efforts for most at-risk populations.

\section{Brief History of HIV Surveillance in Viet Nam}

HIV testing started in Viet Nam in 1988 in four large cities: Ha Noi, Hai Phong, Da Nang and Ho Chi Minh City and included all provinces in 1996. The HIV sentinel surveillance (HSS) system commenced in 10 provinces in 1994 to monitor the HIV seroprevalence annually among people who inject drugs (PWID), female sex workers (FSWs), men who have sex with men (MSM), sexually transmitted infection (STI) patients, antenatal women and army recruits. ${ }^{1}$ Over time HSS was expanded to more provinces and in 2014 was completed in 40 provinces.

In 2000 and 2001, behavioural surveillance surveys were conducted to monitor behaviour of PWID and FSWs in five large provinces, ${ }^{2}$ and Integrated Behavioural and Biological Surveys (IBBS) were conducted in 2005-2006, 2009 and 2013 to measure the prevalence of HIV, other STIs and key HIV risk and preventive behaviour among PWID, FSWs and MSM. ${ }^{3-5}$ Although IBBS provided rich and rigorous data, it is costly and time-consuming to conduct the data collection, analysis, report preparation and dissemination of results.

\section{New Approach in HIV Sentinel Surveillance}

For more timely data that can be readily used, Nha Trang Pasteur Institute in collaboration with the World Health Organization piloted the feasibility of a brief behavioural questionnaire added to HSS among PWID, FSWs and MSM in 2009. Since 2010, the National Institute of Hygiene and Epidemiology has continued this pilot to determine its feasibility and practicality, to monitor trends of key indicators of risk behaviour and to assess coverage of essential HIV/AIDS services. The system became known as HSS $+{ }^{6}$ The positive experiences of the pilot provinces led to an expansion to 12 provinces in 2011, 29 provinces in 2012 and 23 provinces in 2013.

Each HSS + survey comprises sample sizes from 150 to 300, with multistage cluster sampling applied to recruit participants. Clusters were identified through residential communes for PWID and hotspots for FSWs and MSM. Eligible participants were asked to consent to a brief questionnaire and a blood draw and were offered a referral to the local HIV/AIDS services. The questionnaire was simple, covering all global and national

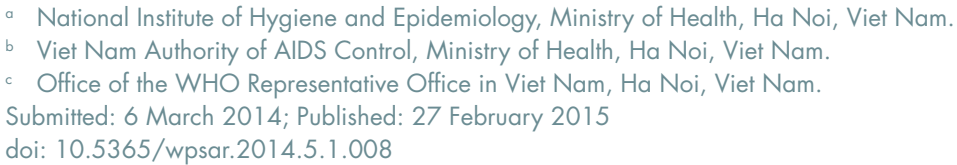


indicators (Table 1). A Standard Operating Procedures was developed, ${ }^{7}$ and provincial AIDS centres' staff were trained on data collection and reporting. An Epi-Data file and report template were provided to each provincial AIDS centre for data entry and report writing.

\section{Key Findings of HSS+}

As a result of the new HSS+, individual provincial reports were submitted within one month, and the national report was completed within two months of data collection. Additional funding (US\$ 8000 per province, which is $10 \%$ of IBBS funding) was provided for the additional fieldwork time needed by HSS staff. Acceptance by the participating populations has been high according to the interviewers. Despite this, the routine field monitoring and technical assistance revealed that there was a lack of adherence to national protocols by local staff in sampling, participant screening and interviewing; following time frame and skip patterns may affect the quality of the data. Supervision of staff is variable and provincial staff capacities differ.

In 2010, 2011 and 2012, respectively, 7\%, 3\% and $2 \%$ of FSWs reported injecting drugs. Of these, $82 \%, 81 \%$ and $70 \%$ reported having injected drugs in the past month. HIV prevalence among drug-injecting FSWs was $29 \%, 22 \%$ and $16 \%$ compared with the HIV prevalence among all FSWs at $4 \%, 3 \%$ and $3 \%$. This disproportionate risk of infection among FSWs who inject drugs resulted in the expansion of needle and syringe access for FSWs through peer outreach programmes. $^{8}$
From HSS+ data it also was found that among 1399 MSM participants in An Giang, Da Nang, Ha Noi, Hai Duong and Ho Chi Minh City in 2011, 3\% of MSM respondents reported injecting drugs in the last month, and $19 \%$ reported selling sex in the past 12 months. These groups had an HIV prevalence of $7 \%$ and $27 \%$, respectively. This diversity of risk among the MSM population led to new approaches and a refocusing of prevention efforts for MSM: strengthening HIV voluntary counselling and testing with focus on MSM who sell sex; needle and syringe programmes; and methadone mainternance treatment for drug-injecting MSM. ${ }^{8}$

\section{Conclusions}

HSS + offers a reasonably cost effective approach to obtaining data on HIV risk behaviour within the standard HSS system. The richness and timeliness of the data for monitoring and evaluating provincial programmes is generating high-level interest. A comparison between HSS + and IBBS will not be able to establish one survey as more valid than the other; however, the timeliness and utility of HSS + data have crystallized support by a donor community anxious for timely data.

\section{Next Steps}

Viet Nam is revising the HSS+ protocol to enhance sampling and improve supervision. As provincial staff come to a better understanding of how the data can help their efforts in prevention and control of HIV in their locality, their receptiveness to protocols should increase. With the HSS + approach in Viet Nam, we can monitor

Table 1. UNGASS and national indicators for HIV/AIDS monitoring and evaluation programme

\begin{tabular}{|c|c|c|}
\hline Indicators & UNGASS indicator $^{9}$ & National indicator \\
\hline FSWs using condoms with the most recent client & $x$ & $x$ \\
\hline FSWs using condoms regularly/consistently with clients in the past month & & $x$ \\
\hline FSWs injecting drug in the past month & & $x$ \\
\hline PWID sharing needles and syringes in the past month & $\mathrm{x}$ & $\mathrm{x}$ \\
\hline PWID using condoms during the most recent sexual intercourse & $\mathrm{x}$ & $x$ \\
\hline MSM using condoms during the most recent sexual intercourse with male partner & $x$ & $x$ \\
\hline PWID using clean needles and syringes in the most recent injection & $x$ & $x$ \\
\hline $\begin{array}{l}\text { FSWs, PWID and MSM being approached by the harm reduction prevention } \\
\text { programmes in the last six months }\end{array}$ & $x$ & $x$ \\
\hline FSWs, PWID and MSM being examined and treated for STIS & & $\mathrm{x}$ \\
\hline FSWs, PWID and MSM being tested and known the results in the past 12 months & $x$ & $x$ \\
\hline
\end{tabular}

FSW, female sex worker; MSM, men who have sex with men; PWID, people who inject drugs; STI, sexually transmitted infection; and UNGASS, United Nations General Assembly Special Session on HIV/AIDS. 
progress in more provinces on an annual basis for a relatively low cost.

\section{Conflicts of interest}

None declared.

Funding

None.

\section{References:}

1. Decision No. 1418: Guideline on HIV/STI surveillance in Viet Nam [In Vietnamese]. Ha Noi, Ministry of Health, 2000 (http://www.vaac.gov.vn:80/Desktop.aspx/Van-ban-phap-quy/ Bo-Y-te/C6D150DEDBA5467CA2F0DC5F05AEC092/, accessed 10 February 2015).

2. Results from the Behavioral Surveillance Survey (BBS) in Viet Nam. Ha Noi, Viet Nam Administration for AIDS Control, Ministry of Health, 2002.

3. Results from the HIV/STI Integrated Biological and Behavioral Surveillance in Viet Nam. Ha Noi, Viet Nam Administration for AIDS Control, Ministry of Health, 2007 (http://www.aidsdatahub.org/sites/default/files/documents/ Results_from_the_from_the_IBBS_in_Vietnam_2006.pdf.pdf, accessed 10 February 2015).

4. National Institute of Hygiene and Epidemiology. Results from the HIV/STI Integrated Biological and Behavioral Surveillance in
Viet Nam - Round II. Ha Noi, Ministry of Health, 2011 (http:// www.nihe.org.vn/new-en/chuong-trinh-phong-chong-sot-xuathuyet-8592614297/2744/Results-from-the-HIVSTI-integratedbiological-and-behavioral-surveillance-IBBS-in-Vietnam--RoundII--2009.vhtm, accessed 10 February 2015).

5. National Institute of Hygiene and Epidemiology. Results from the HIV/STI Integrated Biological and Behavioural Surveillance in Viet Nam - Round IIsI (Draft). Ha Noi, Ministry of Health, 2014.

6. Circular No 09: Guideline on HIV/STI surveillance in Viet Nam [In Vietnamese]. Ha Noi, Vietnam Administration for AIDS Control, Ministry of Health, 2012 (http://www. vaac.gov.vn:80/Desktop.aspx/Van-ban-phap-quy/Bo-Yte/B49D8A7D38BB47938E82CE609959BC04/, accessed 10 February 2015).

7. National Institute of Hygiene and Epidemiology. Standard operating procedures for HIV/STI sentinel surveillance plus behavioral component in Vietnam [In Vietnamese]. Ha Noi, National Institute of Hygiene and Epidemiology, 2014 (http://www. nihe.org.vn/uploads/SOP-HSS-NIHE-9June14-final.pdf, accessed 10 February 2015).

8. National strategy for HIV/AIDS prevention and control to 2020 and vision to 2030 [In Vietnamese]. Ha Noi, Government Cabinet, 2012 (http://www. nihe.org.vn/new-vn/chuong-trinh-giam-sat-theodoi-va-danh-gia-nhiem-h/2876/Chien-luoc-quoc-gia-Phongchong-HIVAIDS-den-nam-2020-va-tam-nhin-2030.vhtm, accessed 10 February 2015).

9. New UNGASS Guidelines for 2010 reporting. Geneva, UNAIDS, 2009 (http://data.unaids.org/pub/Manual/2009/JC1676_Core Indicators_2009_en.pdf, accessed 10 February 2015). 


\title{
Sexually transmitted infections among transgender people and men who have sex with men in Port Vila, Vanuatu
}

\author{
Vanessa Veronese, ${ }^{a}$ Caroline van Gemert, ${ }^{a}$ Siula Bulu, ${ }^{b}$ Tamara Kwarteng, ${ }^{c}$ Isabel Bergari, ${ }^{a}$ Steven Badman, ${ }^{d}$ \\ Alyce Vella and Mark Stoovéae \\ Correspondence to Mark Stoové (e-mail: stoove@burnet.edu.au).
}

Despite high sexually transmitted infection (STI) prevalence in the Pacific, there are limited data on STIs and risk among men who have sex with men (MSM) and transgender people (TG). In 2011, an Integrated Bio-Behavioural Survey recruited self-identified MSM and TG in Port Vila, Vanuatu. Descriptive findings were stratified by sexuality. Among 28 (55\%) MSM and 23 (45\%) TG, recent anal sex with male partners was more common among MSM (94\% vs $71 \% ; P<0.1)$, including with casual ( $47 \%$ vs $35 \%$ ), regular ( $59 \%$ vs $29 \%$ ) and paying partners ( $28 \%$ vs $12 \%$ ). MSM more commonly reported lifetime $(P<0.01)$ and recent sex with female partners $(P<0.01)$. Reported condom use with any partner type was low. More MSM (35\%) than TG (24\%) were diagnosed with an STI; previous treatment-seeking behaviour when symptomatic was lower among TG $(P<0.1)$. Tailored strategies acknowledging differences between MSM and TG are required to reduce STI vulnerability in Vanuatu.

$\mathrm{D}$ espite a high prevalence of sexually transmitted infections (STIS) in Pacific island countries and territories, ${ }^{1-4}$ there are limited data on STIS and risk behaviour among men who have sex with men (MSM) and transgender people (TG). While culturally defined TG roles for men exist throughout Pacific island countries and territories, ${ }^{5}$ male-to-male sexual contact occurs among men who do not identify as TG or homosexual. In Vanuatu, up to $10 \%$ of young males reported sex with a male partner. ${ }^{6}$ Stigma and discrimination towards sexual minorities deters disclosure of sexuality and male-tomale sexual behaviour. This can result in low levels of STI testing and diagnosis, underreporting of risk behaviours and limited engagement with prevention and treatment services. ${ }^{5,7-9}$

The limited data for MSM and TG populations in Pacific island countries and territories hamper risk reduction among these groups and their sexual partners. In 2011, an Integrated Bio-Behavioural Survey of selfidentified MSM and TG was conducted by the Burnet Institute in partnership with Wan Smolbag and the Vanuatu Ministry of Public Health in Port Vila, Vanuatu.

\section{METHODS}

\section{Recruitment and data collection}

Respondent-driven sampling (RDS) was used to recruit participants between November 2011 and April 2012. Biological men, identifying as male or transgender women, and aged over 18 years who reported anal sex with another male in previous year were eligible.

Seven seeds (initial recruits) were recruited through a local nongovernmental organization and chosen for their close linkages with the study population and for diversity (e.g. gender, age, marital status). Seeds were given three coupons to recruit the first wave of participants who were also provided three coupons to recruit the next wave, and so on; willing participants then presented for data collection.

Three field researchers assessed eligibility and obtained informed verbal consent. Interviews were conducted in the local language; the questionnaire included items about socio-demographics, sexual risk

\footnotetext{
Center for Population Health, Burnet Institute, Melbourne, Australia.

Wan Smolbag Theatre, Port Vila, Vanuatu.

Center for International Health, Burnet Institute, Melbourne, Australia

Kirby Institute, University of South Wales, Sydney, Australia.

Department of Epidemiology and Preventive Medicine, Monash University, Melbourne, Australia.

Submitted: 10 February 2014; Published: 5 March 2015

doi: 10.5365/wpsar.2014.5.1.001
} 
behaviours, previous diagnoses of any STI, prior STI symptoms and treatment-seeking behaviour. Participants were then referred for pre-test counselling and STI testing. Nurses collected 20 millilitres of venous blood for HIV and syphilis testing and rectal swabs for chlamydia and gonorrhoea testing. Chlamydia and gonorrhoea tests were performed using BD ProbeTec (Becton, Dickinson and Company, New Jersey, USA) nucleic acid amplification testing; tests for active syphilis used RPR screening confirmed through Determine ${ }^{\mathrm{TM}}$ Syphilis TP (Inverness Medical, Bedford, UK). For HIV, a rapid screening test (Determine ${ }^{\mathrm{TM}}$ ) was used with confirmation of reactive samples by two additional rapid tests (Insti, BioLytical, British Columbia, Canada and Unigold, Trinity Biotech, Carlsbad, USA).

\section{Analysis}

Most variables were dichotomous or categorical; these were analysed using Fisher's exact test. For numerical variables, the median and interquartile ranges were calculated. Due to the small sample size and limited recruitment, the data could not be analysed using methods for RDS. Analyses were conducted using Epilnfo and StataSE v11.

To determine recent STI exposure, participants were asked if they had experienced any of four STI symptoms in the previous 12 months: (1) genital discharge from the vagina; (2) genital ulcer or sore on the vagina; (3) burning or sharp pain on urination; and (4) rash or genital itching. Participants were then classified as self-reporting symptoms or not during this time period. Consistent condom use was defined as always using a condom with partner during the previous six months.

The Ministry of Health, Government of the Republic of Vanuatu Ethics Committee and the Alfred Hospital Ethics Committee approved this study. No identifying information was collected from participants.

\section{RESULTS}

Six seeds recruited 45 participants (total $n=51$ ); 23 (45\%) identified as TG and 28 (55\%) as MSM. An additional 20 MSM refused to participate, citing fear of disclosure of sexuality or an unwillingness to test for HIV and STIs. The most common age group was $20-29$ years; $21 \%$ of MSM and $17 \%$ of TG were aged 19 years or under.

TG participants reported younger age at sexual debut compared to MSM (16 or younger $(P<0.05$ ). Condom use at first sex was low among all respondents. While a history of forced sex was high overall (47\%), it was more commonly reported among TG $(P<0.1)$ (Table 1).

The majority of participants reported sexual contact with a male partner during the past six months. Consistent condom use during anal sex was low across all regular, casual, paying and paid sexual partners (Table 1). We also found evidence of multiple and potentially overlapping sexual partnerships; among the eight MSM reporting regular partners in the past six months, three also reported paid partners, three reported casual partners, one reported a paying partner and four reported female partners.

Significantly more MSM reported sex with a female partner compared to TG $(P<0.01)$; the majority of MSM reported sex with a female during the past six months. Consistent condom use with recent female partners was low overall (Table 1).

Approximately one in five MSM and no TG had a history of STI diagnosis $(P=0.03)$, and one third of MSM and one in five TG reported genital symptoms in the past year. MSM reported higher levels of genital symptoms across all four types investigated and were also significantly more likely to seek treatment for these symptoms $(P=0.02)$ (Table 1$)$.

For the 43 participants that provided a sample, $24 \%$ of TG and $35 \%$ of MSM tested positive for any STI; chlamydia was detected in $19 \%$ of samples and gonorrhoea in $9 \%$. Of the 39 participants tested for syphilis, $3 \%$ were positive (Table 1 ). There were no detected cases of HIV.

\section{DISCUSSION}

While acknowledging the limitations in our sample size, these findings nonetheless advance our understanding 
Table 1. Socio-demographic and self-reported sexual risk and STI exposure and treatment history by reported gender $(n=51)$

\begin{tabular}{|c|c|c|c|}
\hline & TG $(n=23)$ & MSM $(n=28)$ & $p$-value \\
\hline Socio-demographic & $\mathrm{n}(\%)$ & n (\%) & \\
\hline Age group (years) & & & 0.235 \\
\hline 19 and below & $4(17)$ & $6(21)$ & \\
\hline $20-29$ & $9(39)$ & $16(57)$ & \\
\hline 30 and above & $10(43)$ & $6(21)$ & \\
\hline Median age (range) & $26(18-48)$ & $25(18-46)$ & \\
\hline \multicolumn{4}{|l|}{ Sexual risk } \\
\hline Age at first sex & & & 0.03 \\
\hline 16 and below & $17(74)$ & $10(36)$ & \\
\hline $16-19$ & $4(17)$ & $12(43)$ & \\
\hline 20 and above & $2(9)$ & $6(21)$ & \\
\hline Median age at first sex (range) & $12(7-22)$ & $16(12-28)$ & \\
\hline Condom use at first sex & $2(9)$ & $4(14)$ & - \\
\hline Forced sex ever & $14(61)$ & $10(36)$ & 0.07 \\
\hline \multicolumn{4}{|l|}{ Male partners } \\
\hline Any sexual contact with male partner (past six months) & $17(74)$ & $18(64)$ & \\
\hline Anal sex & $12(71)$ & $17(94)$ & 0.06 \\
\hline Oral sex & $17(100)$ & $13(72)$ & 0.02 \\
\hline Median number of partners (range) & $2(0-12)$ & $4(0-21)$ & \\
\hline Regular partner & $6(35)$ & $8(44)$ & \\
\hline Median number of regular partners (range) & $3(2-8)$ & $3(1-6)$ & \\
\hline Consistent condom use with regular partner & 0 & $1(12)$ & \\
\hline Casual partner & $5(29)$ & $10(56)$ & \\
\hline Median number of casual partners (range) & $7(2-20)$ & $2(1-4)$ & \\
\hline Consistent condom use with casual partner & 0 & 0 & \\
\hline Received payment for sex & $2(12)$ & $5(28)$ & \\
\hline Median number of casual partners (range) & $2(1-2)$ & $1(1-2)$ & \\
\hline Condom use at last sex with paying partner & 0 & $3(60)$ & \\
\hline Paid for sex last 12 months & 1 & 3 & \\
\hline Median number of paid partners (range) & 1 & $2(1-4)$ & \\
\hline Condom use at last sex with paid partners & 0 & 0 & \\
\hline \multicolumn{4}{|l|}{ Female partners } \\
\hline Female sex ever & $7(30)$ & $24(86)$ & $<0.01$ \\
\hline Female sex last six months & $5(71)$ & $21(88)$ & 0.31 \\
\hline Median number of female partners (range) & $1(0-3)$ & $2(0-11)$ & \\
\hline Consistent condom use with female partner & $1(20)$ & $1(5)$ & \\
\hline \multicolumn{4}{|l|}{ STI exposure and treatment seeking history } \\
\hline Ever diagnosed with a STI & 0 & $5(18)$ & 0.03 \\
\hline Genital symptoms past 12 months & $4(17)$ & $10(36)$ & \\
\hline Sought treatment for these symptoms & 0 & $7(70)$ & 0.02 \\
\hline at $\mathrm{KPH}$ clinic & 0 & $5(71)$ & \\
\hline at hospital & 0 & $1(14)$ & \\
\hline at other location & 0 & $1(14)$ & \\
\hline STI diagnosis & TG $(n=19)$ & MSM $(n=24)$ & \\
\hline Chlamydia & $3(16)$ & $5(21)$ & \\
\hline Gonorrhoea & $2(11)$ & $2(8)$ & \\
\hline Syphilis* & 0 & $1(5)$ & \\
\hline HIV & 0 & 0 & \\
\hline Any STI diagnosed $^{\dagger}$ & $4(24)$ & $7(35)$ & \\
\hline
\end{tabular}

MSM, men who have sex with men; STI, sexually transmitted intection; TG, transgender.

* Denominator $\mathrm{TG}=18 ; \mathrm{MSM}=21$

+ Denominator TG $=17$; MSM $=20$ 
of the sexual health vulnerabilities for MSM and TG in Vanuatu, a region characterized by distinctive and culturally defined sexualities.

The detection of STIs in one in five TG and one in three MSM represents comparably high prevalence for Pacific island countries and territories., ${ }^{1,3}$ No TG and only five MSM reported an STI diagnosis history. That $17 \%$ of TG and $36 \%$ of MSM reported STI symptoms in the past 12 months suggests low treatment-seeking behaviour, particularly among symptomatic TG and may reflect barriers to accessing treatment. In settings where same-sex behaviour is not culturally permissible, TG may be unwilling to access mainstream sexual health services where they might be asked to disclose sexual behaviours. ${ }^{10}$ However, services specifically targeting gay men may not appeal to bisexual MSM who maintain relationships with women and thus present a barrier to care and preventative treatment among this group. Our findings underscore the needs for culturally appropriate health promotion and sexual health services that reflect the specific needs of MSM and TG populations.

We found consistently low levels of condom use with regular and casual partners. Lifetime and recent sex with females by MSM was also higher than reported elsewhere. ${ }^{1,9}$ Against a background of high STI prevalence, low levels of condom use with female partners, high levels of bisexual risk behaviours and multiple and potentially overlapping male and female sexual partnerships create a potential bridge between high-risk groups and the general population. It is crucial that approaches to preventing and treating STIs in Vanuatu incorporate discrete strategies for partner notification and treatment for female partners.

This study was subject to limitations which must be considered when reviewing the findings. Our small sample size limited the detection of particular outcomes and statistical power that may have been observed in a larger sample. The RDS strategy employed also did not result in sufficient recruitment. Our question about participant's age at sexual debut implied but did not specify homosexual sex; thus, our findings may not accurately represent the age of homosexual debut among participants.

Despite this, our results show important risk behaviours and profiles of TG and MSM in Vanuatu not previously documented. Overall, high and somewhat specific levels of risk and vulnerability among TG and MSM in Vanuatu were determined. Our findings underscore the need for comprehensive and targeted STI treatment and prevention services for MSM and tailored health promotion strategies that recognizes different sexual identities and adequately engages non-gay identifying MSM and their female partners in Vanuatu.

\section{Conflicts of interests}

None declared.

\section{Funding}

Funding was provided by the Pacific Island HIV and STI Response Fund grant, a collaborative funding mechanism by the Australia and New Zealand governments and managed by the Secretariat of the Pacific Community.

\section{Acknowledgements}

We wish to thank the Vanuatu Ministry of Health for its ongoing support and for laboratory testing; National Serology Reference Laboratory (NRL, Melbourne, Australia) for laboratory testing; Wan Smolbag for coordinating data collection and entry and management of the field researchers; WHO Western Pacific Regional Office and the Pacific UNAIDS Coordinator for their technical support in project implementation. Caroline van Gemert received a Travel Grant from the lan Potter Foundation for training in RDS. Laboratory testing of specimens at the Port Vila Central Hospital was provided by the Global Fund Round 7 Grant through the Vanuatu Ministry of Health. Finally, we thank all participants of the study for their valuable input. We would also like to thank William Craw for his valuable advice.

\section{References:}

1. Rawstorne P et al. Shattered Stereotypes: Twists \& Turns in Sexual Practices. An integrated bio-behavioural survey of transgender and men who have sex with men in Suva and Lautoka, Fiji. Suva, MEN Fiji, 2012.

2. Rawstorne P, Te Tiare Association of the Cook Islands, Secretariat of the Pacific Community. Second Generation Surveillance Survey of Akavaine and MSM in Cook Islands 2009. Cook Islands, Secretariat of the Pacific Community, 2009.

3. Second Generation Surveillance Survey of MSM in Tonga 2008. Tonga, Ministry of Health, 2008.

4. Second Generation Surveillance: Behavioural survey among MSM. Guam, Guam Department of Public Health and Social Services, 2007. 
5. Buchanan-Aruwafu H. An Integrated Picture: HIV risk and vulnerability in the Pacific - Research Gaps, Priorities and Approaches. New Caledonia, Secretariat of the Pacific Community, 2007.

6. I No bin gat protection. Understanding HIV and AIDS risk and vulnerability among Vanuatu Youth. Suva, United Nations Internation Children's Fund Pacific Sub-Regional Office, 2010.

7. HIV Surveillance in Pacific Island Countries and Territories: 2012 report. New Caledonia, Surveillance and Operational Health Research Team, Secretariat of the Pacific Community, 2013.
8. Godwin J. Legal environments, human rights and HIV responses among men who have sex with men and transgender people in Asia and the Pacific: An agenda for action. Bangkok, United Nations Development Programme, 2010.

9. Bavington B et al. Secret Lives, Other Voices... A communitybased study exploring male-to-male sex, gender identity and HIV transmission risk in Fiji. Suva, AIDS Task Force of Fiji, 2011.

10. Sullivan PS et al. Successes and challenges of HIV prevention in men who have sex with men. Lancet, 2012, 380:388-389. doi:10.1016/S0140-6736(12)60955-6 pmid:22819659 


\title{
Identification of Enterovirus C105 for the first time in New Zealand
}

\author{
Angela Todd, a Susan Taylor ${ }^{b}$ and Q Sue Huang ${ }^{a}$ \\ Correspondence to Angela Todd (e-mail: angela.todd@esr.cri.nz).
}

We report on the first identification of Enterovirus C105 (EV-C105) in New Zealand from a 52-year-old male hospitalized with mild respiratory tract symptoms. Enterovirus genotyping was performed by partial sequencing of the VP1 region of the enterovirus genome. This highlights the importance of enterovirus surveillance for detection of the importation of new genotypes such as EV-C105, thus allowing a better understanding of the roles they play in disease.

$\mathrm{T}$ he New Zealand National Poliovirus and Enterovirus Identification Reference Laboratory at the Institute of Environmental Science and Research (ESR) Limited, National Centre for Biosecurity and Infectious Disease routinely receives untyped enterovirus clinical specimens or cell culture isolates from four major hospitals (based in Auckland, Waikato, Wellington and Christchurch) as part of the New Zealand enterovirus surveillance network. This surveillance network started in the 1980s and on average receives 150 specimens per year. The aims of this network are (1) to identify the circulation patterns of enterovirus genotypes and predominant strains; (2) to describe clinical diseases associated with circulating genotypes; (3) to detect enterovirus outbreaks to assist with public health intervention; and finally (4) to supplement the national poliovirus surveillance programme. The four laboratories in conjunction with ESR service the entire population of New Zealand. Enterovirus surveillance operates year-round and is based on reports from routine diagnostic services for patients. Each week, all enterovirus identification results are reported to ESR and then collated and published nationally. ${ }^{1}$ In addition, untyped enteroviruses are referred to ESR for further identification.

Enterovirus C105 (EV-C105) is a member of the human enterovirus group $\mathrm{C}$ species and was first identified in the Democratic Republic of Congo in late 2010 in a faecal sample collected from a fatal acute flaccid paralysis patient during a poliomyelitis outbreak. ${ }^{2}$ Due to a lack of sequence data this virus was initially identified as Enterovirus 109 (EV-109). ${ }^{3}$ In 2012, the virus was assigned as EV-C105 because subsequent sequencing showed that it had only $74.7 \%$ nucleotide and $82.5 \%$ amino acid identity to EV-109, thus satisfying the criteria for assignment of a new enterovirus serotype. ${ }^{4}$ Since then, EV-C105 has also been identified in a patient in Peru and in another patient in Cyprus, both with respiratory disease. ${ }^{5,6}$ This suggests a global distribution although the overall incidence appears to be very low. A greater awareness of EV-C105 may help to detect this virus with increased frequency and to find out more about the pathogenic role and disease association of this particular genotype.

Here we report on the identification of EV-C105 for the first time in New Zealand from a patient with mild lower respiratory tract symptoms during an admission to a hospital in Auckland through the national enterovirus surveillance system.

\section{CASE REPORT}

A 52-year-old Maori male was admitted to a hospital in Auckland, New Zealand in October 2013. Unrelated to the reason for presenting to the hospital, cough and wheeze were noted on the second day of his admission and a nasopharyngeal swab for respiratory virus testing was collected. The patient remained

Institute of Environmental Science and Research Limited, National Centre for Biosecurity and Infectious Disease, Wallaceville, Upper Hutt, New Zealand.

Middlemore Hospital, Otahuhu, Auckland, New Zealand

Submitted: 23 October 2014; Published: 10 February 2015

doi: 10.5365/wpsar.2014.5.4.003 
afebrile. The nasopharyngeal swab tested negative for influenza, adenovirus, respiratory syncytial virus, human metapneumovirus, parainfluenza viruses $1-3$ and rhinovirus. ${ }^{7}$ Enteroviral RNA was detected using realtime reverse transcription polymerase chain reaction. ${ }^{8}$ A partial VP1 sequence was then obtained by amplification and sequencing of a $375 \mathrm{bp}$ region. ${ }^{9}$ The sequence was compared to known VP1 sequences in GenBank using tblastx (which searches a translated nucleotide database using a translated nucleotide query). ${ }^{10}$ It showed $99 \%$ amino acid identity to EV-C105 with sequence coverage of $100 \%$ (GenBank accession number: AFG25720), an EV-C105 strain that was identified in Cyprus in 2012. The next closest genotype was EV-109 with 89\% amino acid identity (GenBank accession number: ADK22861), another group $\mathrm{C}$ enterovirus.

\section{DISCUSSION}

We have reported the identification of EV-C105 for the first time in the Asia-Pacific region from a 52-year-old male with mild lower respiratory tract symptoms. EV-C105 has been identified only rarely in various countries including the Democratic Republic of Congo, Peru and Cyprus. Prior to detecting the EV-C105 case in New Zealand, EV-C105 has been previously identified in a faecal sample from an acute flaccid paralysis patient and respiratory samples taken from paediatric patients. The identification of newly-identified enteroviruses such as EV-C105 illustrates the importance of ongoing enterovirus surveillance in allowing the identification of emerging and re-emerging enterovirus genotypes and has the potential to inform public health interventions. Increased awareness of EV-C105 may allow for improved detection of the virus; however, further analysis is required to determine the pathogenic role, disease association, mode of transmission, global occurrence and public health implications of this rare enterovirus.

\section{Conflicts of interest}

None declared.

\section{Funding}

The national poliovirus/enterovirus surveillance is funded by the New Zealand Ministry of Health, which kindly permitted the use of relevant data for publication.

\section{Acknowledgements}

We wish to acknowledge W Allan Nix, research microbiologist in the Picornavirus Laboratory at the Centers for Disease Control and Prevention, for his assistance with the enteroviral typing analysis and review of this paper.

\section{References:}

1. Public Health Surveillance [Internet]. Information for New Zealand Public Health Action (https://surv.esr.cri.nz/virology/ virology_weekly_report.php, accessed 20 November 2014).

2. Grard $\mathrm{G}$ et al. Type 1 wild poliovirus and putative enterovirus 109 in an outbreak of acute flaccid paralysis in Congo, October-November 2010. Eurosurveillance: European Communicable Disease Bulletin, 2010, 15(47):pii:19723. pmid:21144443

3. Lukashev AN et al. Novel serotypes 105 and 116 are members of distinct subgroups of human enterovirus C. The Journal of General Virology, 2012, 93:2357-2362. doi:10.1099/vir.0.043216-0 pmid:22894922

4. Oberste MS et al. Molecular evolution of the human enteroviruses: correlation of serotype with VP1 sequence and application to picornavirus classification. Journal of Virology, 1999, 73:19411948. pmid: 9971773

5. Richter $\mathrm{J}$ et al. Newly emerging $\mathrm{C}$ group enteroviruses may elude diagnosis due to a divergent 5'-UTR. International Journal of Infectious Diseases, 2013, 17:e1245-1248. doi:10.1016/j. ijid.2013.07.010 pmid:24080070

6. Tokarz R et al. Genomic analysis of two novel human enterovirus $C$ genotypes found in respiratory samples from Peru. The Journal of General Virology, 2013, 94:120-127. doi:10.1099/ vir.0.046250-0 pmid:23034595

7. Centers for Disease Control and Prevention (CDC). CDC Real-Time RT-PCR Assays for Non-Influenza Respiratory Viruses. Atlanta, Centers for Disease Control and Prevention, 2010.

8. Oberste MS et al. Comparative evaluation of Taqman real-time PCR and semi-nested VP1 PCR for detection of enteroviruses in clinical specimens. Journal of Clinical Virology, 2010, 49:73-74. doi:10.1016/j.jcv.2010.06.022 pmid:20667767

9. Nix WA, Oberste MS, Pallansch MA. Sensitive, seminested PCR amplification of VP1 sequences for direct identification of all enterovirus serotypes from original clinical specimens. Journal of Clinical Microbiology, 2006, 44:2698-2704. doi:10.1128/ JCM.00542-06 pmid: 16891480

10. Altschul SF et al. Basic local alignment search tool. Journal of Molecular Biology, 1990, 215:403-410. doi:10.1016/S00222836(05)80360-2 pmid:2231712 


\title{
International Health Regulations (2005) facilitate communication for in-flight contacts of a Middle East respiratory syndrome case, Hong Kong Special Administrative Region, 2014
}

\author{
Poon Kwok-ming, ab Wong Miu-ling, ab Leung Yiu-hong, a Sin Ka-wai, a To May-kei Liza a and Chuang Shuk-kwan ${ }^{a}$ \\ Correspondence to Poon Kwok-ming (e-mail: mo_fetpl@dh.gov.hk).
}

The International Health Regulations (IHR) (2005) require World Health Organization Member States to notify events fulfilling two of four criteria: (1) serious public health impact; (2) unusual or unexpected event; (3) significant risk of international spread; or (4) significant risk of international travel or trade restrictions. ${ }^{1}$ In-flight transmission of infections like severe acute respiratory syndrome is well documented. ${ }^{2}$ With the enormous amount of air travel today, the risk of increasing in-flight transmission and subsequent international spread of infections are increasing. Prompt notification and information sharing under the IHR mechanism is critical for effective contact tracing and prompt control measures. We report on a case of in-flight exposure to an infection with significant public health risks that was successfully resolved using IHR (2005) guidelines.

$\mathrm{T}$ he government of the Hong Kong Special Administrative Region launched the Preparedness Plan for Middle East Respiratory Syndrome (MERS) in June 2014, upholding established principles of rapid control of disease transmission and responsive risk communication. ${ }^{3}$ It sets out a multidisciplinary disease control framework involving the Department of Health, Hospital Authority, the travel industry and airlines, etc. Under the plan, the Centre for Health Protection of the Department of Health developed protocols for case and contact management. Its risk-based classifications of "close" and "other" contacts determine subsequent public health actions, including quarantine/travel restrictions for close contacts, medical surveillance, referral, isolation and laboratory investigation.

On 2 October 2014, the World Health Organization (WHO) Western Pacific Regional Office informed through the IHR mechanism of Hong Kong Special Administrative Region that a Chinese national, whose point of origin was Hong Kong Special Administrative Region, sat near a MERS case on a flight from Qatar to Austria. Subsequent investigations revealed that a Hong Kong Special Administrative Region tour group of 42 members was on the same flight. We report investigations and public health measures for these in-flight contacts while they were still on their journey in Europe.

\section{METHODS}

We obtained information on the index case and a passenger list detailing points of origin and seat numbers from the WHO Regional Office through IHR communications. Contact tracing was performed according to the Preparedness Plan for MERS whereby "close contacts" were defined as passengers seated in the same row or up to two rows in front of/behind the case, and "other contacts" as other passengers seated in the same cabin or passengers from the same tour group. The airline provided the aircraft seating plan, and the travel agency provided a tour member list. We crosschecked the tour member list with the list provided by the WHO Regional Office and the aircraft seating plan. The health conditions of all contacts were ascertained over the phone and health advice was provided. Using the International Health Regulations (IHR) mechanism, we informed relevant overseas health authorities of

Surveillance and Epidemiology Branch, Centre for Health Protection, Department of Health, Hong Kong Special Administrative Region.

Field Epidemiology Training Programme, Hong Kong Special Administrative Region.

Submitted: 9 January 2015; Published: 26 March 2015

doi: 10.5365/wpsar.2015.6.1.008 
Figure 1. Seating plan of the aircraft from Qatar to Austria

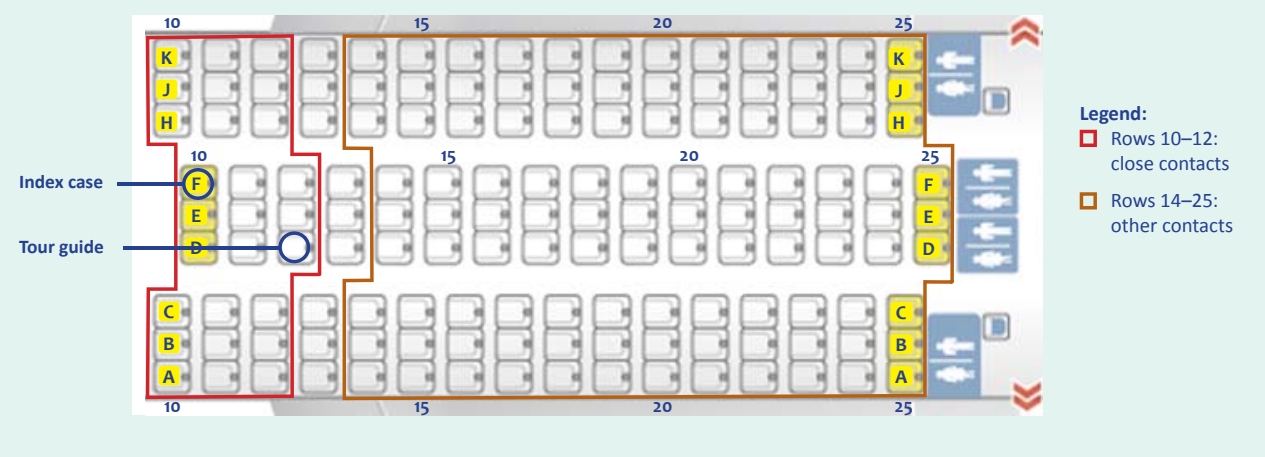

our epidemiological findings and collaborated with them to implement control measures for the contacts. We continued medical surveillance after the contacts had returned to Hong Kong Special Administrative Region and provided risk communications to Hong Kong Special Administrative Region general public.

\section{RESULTS}

The index case was a citizen of Saudi Arabia who had symptoms of upper respiratory tract infection and fever from 17 September 2014. She was still symptomatic when she travelled on a flight from Qatar to Austria on 22 September. Austrian health authorities confirmed she was a case of MERS on 29 September. On 2 October, the WHO Western Pacific Regional Office informed through the IHR mechanism of Hong Kong Special Administrative Region that a Hong Kong Special Administrative Region citizen who had been seated within two rows of the index case on the flight. The passenger list included an additional 42 passengers with Hong Kong Special Administrative Region as their points of origin.

We classified the Hong Kong Special Administrative Region citizen (seat 12D) as a close contact (within two rows of index case: seat 10F); he was the tour guide of the 42 other Hong Kong Special Administrative Region passengers. Twenty of these passengers were seated in the same cabin as the index case, however all 42 tour members were managed as other contacts (Figure 1). All contacts were travelling through Europe and had reached Poland on 1 October. On 2 October (Day 10 of last exposure from the flight), we ascertained that none had been sick since 22 September. We advised the tour guide to minimize contact with the group and for the whole group to wear face masks during their journey. We issued a press release on 2 October.

We alerted Poland health authorities on 2 October to the whereabouts of the tour group. The return flight of the tour guide was postponed to 6 October (Day 14 - maximum incubation period of MERS) to avoid potential risk to other travellers in case the tour guide developed symptoms during his flight back to Hong Kong Special Administrative Region. Poland health authorities conducted medical surveillance of the tour guide who voluntarily stayed in a hotel in Poland until 6 October. He did not develop symptoms.

The 42 tour members boarded the scheduled return flight departing from Poland on 3 October. With assistance from the airline, we arranged for them to sit at the back of the aircraft and stay on board after arrival at Hong Kong Special Administrative Region for health screening. All were asymptomatic upon return to Hong Kong Special Administrative Region. Their health status was monitored by telephone interview until 20 October (Day 28 - twice the maximum incubation period of MERS) with good compliance. A tour member who reported respiratory symptoms on 6 October was promptly isolated; she tested negative for MERS. Through the IHR mechanism, we communicated our actions to Poland and Austria health authorities and other IHR colleagues in Europe.

\section{DISCUSSION}

International air travel of a symptomatic MERS case constitutes a potential risk of international spread of MERScoronavirus and sufficient criteria for IHR notification. ${ }^{1}$ With the successfully triggered IHR mechanism, the WHO 
Regional Office informed through IHR mechanism of Hong Kong Special Administrative Region within three days of diagnosis of the case. This communication facilitated effective contact tracing and prompt implementation of control measures and was timely in preventing the tour guide from boarding the return flight.

Communication under the IHR mechanism has been widely adopted and used in the Western Pacific Region since the endorsement of IHR in 2007, especially regarding information on emerging infectious diseases. The Hong Kong Special Administrative Region has been active regarding notification and information exchange with WHO. ${ }^{4}$ The IHR mechanism has facilitated contact tracing for infectious diseases among Member States in the past. ${ }^{4}$ Well-established channels of communication under IHR have also enabled efficient collaboration between health authorities across continents. In this case, smooth implementation of control measures required a great deal of international coordination. While Poland's health authorities took charge of necessary local measures, we helped to reschedule the tour guide's return flight. This efficient collaboration and timely arrangement stopped him from boarding another international flight, reducing the potential risks if he had in-flight onset of symptoms.

Under the Preparedness Plan for MERS, the response was based on risk-based contact management. Only the tour guide's return flight was postponed until Day 14; the other tour members travelled back to Hong Kong Special Administrative Region as scheduled. The multidisciplinary disease control framework among the Department of Health, the travel industry and airlines allowed for smooth communications and precautionary travel arrangements for the tour members on their return flight and the subsequent return of the tour guide. The preparedness plan also resulted in immediate referral, isolation and testing of the symptomatic tour member after their return, relying on coordinated efforts among the Department of Health, Hospital Authority and the ambulance centre under the Fire Services Department.

Although there are international risk assessment guidelines regarding aircraft transmission of various infectious diseases, ${ }^{5}$ guidelines for emerging infections like MERS are still evolving and differ by country. For instance, the Public Health Agency of Canada considers passengers in the same row and up to three rows in front of/behind as priority, recommending all passengers should be followed up when feasible; ${ }^{6}$ the Health Protection Agency in the United Kingdom defines close contacts as those sitting in the same row and up to two rows in front of/behind. ${ }^{7}$ WHO defines close contacts as those travelling together with a MERS case in any kind of conveyance. ${ }^{8}$ Such variations result in different management of contacts among countries. Despite the small risk to the 42 tour members on board, we took a precautionary approach and managed them all as other contacts, taking into account the uncertainties of unrecognized in-flight exposure in the same cabin and the common practice of seat swapping among tour group members.

We also adopted a more stringent approach than most international guidelines for the length of contact tracing. WHO recommends that close contacts should be monitored for the appearance of respiratory symptoms for 14 days after last exposure; ${ }^{9}$ we decided to extend health monitoring to 28 days, taking into account the potential risks of hidden chains of transmission within the tour group and the availability of our resources. We acknowledged the inter-organizational differences in risk assessment and extent of information sharing and adhered to our protocol. We extended our scope of contact tracing and identified additional contacts by combining all sources of information from WHO, the airline and the tour member list obtained from the travel agency. Fortunately, all other contacts were from the same tour group, greatly facilitating investigation and control measures.

\section{CONCLUSIONS}

Three lessons were learnt from this public health event. First, timely information sharing and communications under IHR mechanisms greatly facilitated our contact tracing and management by allowing efficient collaboration with overseas health authorities and the WHO Regional Office. Second, effective contact tracing enabled prompt control measures for flight rescheduling and precautionary travel arrangements to minimize further disease transmission. Third, a well-established preparedness plan, including a protocol for contact management, ensured efficient management of in-flight contacts of an overseas MERS case. 


\section{Conflicts of interest}

None declared.

\section{Funding}

None.

\section{Acknowledgement}

We would like to thank all staff of the Hong Kong Special Administrative Region Surveillance and Epidemiology Branch of the Centre for Health Protection who contributed in the investigation and control of this outbreak. We would also like to thank Dr Alain Moren and Dr Marta Valenciano for their advice in report writing.

\section{References:}

1. WHO guidance for the use of Annex 2 of the International Health Regulations (2005). Decision instrument for the assessment and notification of events that may constitute a public health emergency of international concern. Geneva, World Health Organization, 2008 (http://www.who.int/ihr/revised_annex2 guidance.pdf, accessed 19 February 2015).

2. Olsen SJ et al. Transmission of the severe acute respiratory syndrome on aircraft. New England Journal of Medicine, 2003, 349:24162422. doi:10.1056/NEJMoa031349 pmid:14681507

3. Press release: Preparedness Plan for Middle East Respiratory Syndrome launched. Hong Kong Special Administrative Region, Centre for Health Protection, Department of Health, 12 June 2014 (http://www.chp.gov.hk/en/view content/35026.html, accessed 24 November 2014).

4. Fearnley E, Li A. International Health Regulations (2005): public health event communications in the Western Pacific Region. Western Pacific Surveillance and Response Journal, 2013, 4:2627. doi:10.5365/wpsar.2013.4.3.003 pmid:24319610

5. ECDC Technical Report: Risk assessment guidelines for infectious diseases transmitted on aircraft. Stockholm, European Centre for Disease Prevention and Control, 2009 (http://www.hpsc.ie/A-Z/ Vectorborne/ViralHaemorrhagicFever/Guidance/File,4661,en.pdf, accessed 19 February 2015).

6. Public Health management of human illness associated with Middle East Respiratory Syndrome Coronavirus (MERSCoV): Interim guidance for containment when imported cases with limited human-to-human transmission are suspected/confirmed in Canada. Ontario, Public Health Agency of Canada, 2013 (http://www.phac-aspc.gc.ca/eri-ire/ coronavirus/guidance-directives/mers-cov-srmo-2-eng.php, accessed 24 November 2014).

7. Health Protection Agency UK Novel Coronavirus Investigation Team. Evidence of person-to-person transmission within a family cluster of novel coronavirus infections, United Kingdom, February 2013. Euro surveillance: European Communicable Disease Bulletin, 2013, 18(11):pii=20427. pmid:23517868

8. Interim surveillance recommendations for human infection with Middle East respiratory syndrome coronavirus (as of July 2014). Geneva, World Health Organization, 2014 (http:// www.who.int/csr/disease/coronavirus infections/InterimRevised SurveillanceRecommendations_nCo-Vinfection_14July2014. pdf?ua $=1$, accessed 24 November 2014).

9. WHO guidelines for investigation of cases of human infection with Middle East Respiratory Syndrome Coronavirus (MERSCoV) (July 2013). Geneva, World Health Organization, 2013 (http://www.who.int/csr/disease/coronavirus_infections/ MERS CoV investigation guideline Jul13.pdf?ua $=1$, accessed 24 Novembèr 2014). 


\title{
Ebola preparedness in the Western Pacific Region, 2014
}

\author{
Xu Zhen, ${ }^{a^{*}}$ Boris Pavlin, ${ }^{b^{*}}$ Raynal C. Squires, ${ }^{a}$ Thilaka Chinnayah, ${ }^{a}$ Frank Konings, ${ }^{a}$ Chin-Kei Lee ${ }^{a}$ and Li Ailan ${ }^{a}$ on \\ behalf of the World Health Organization Regional Office for the Western Pacific Ebola Emergency Support Team \\ Correspondence to Xu Zhen (email: xuz@wpro.who.int).
}

West Africa is currently experiencing the largest outbreak of Ebola virus disease (EVD) in history with intense transmission in several affected countries. For non-affected countries, the best protective measures are adequate levels of preparedness including vigilant surveillance to detect cases early and well-prepared health systems to ensure rapid containment of the virus and to avoid further spread. The World Health Organization Regional Office for the Western Pacific recently conducted two activities: a web-based EVD preparedness survey and an EVD simulation exercise to determine the overall level of EVD preparedness in the Region. The survey and exercise together demonstrate there is a good overall level of preparedness for a potential imported case of EVD in the Western Pacific Region. However, a number of areas still require further strengthening before the Region can efficiently and effectively respond to potential EVD events, including laboratory testing arrangements; clinical management and infection prevention and control; and public health intervention measures, particularly at points of entry. Importantly, the survey and exercise also highlight the unique situation in Pacific island countries and emphasize that special considerations are needed to better support these countries in EVD preparedness.

$\mathrm{T}$ he largest outbreak of Ebola virus disease (EVD) in history has been ongoing in West Africa since December 2013. ${ }^{1}$ Recent transmission of EVD outside of West Africa in the United States of America and Spain ${ }^{2,3}$ suggests that no region of the world is immune to either the introduction or the onward transmission of EVD.

There are no direct flights between the three countries with widespread transmission of EVD in West Africa and any countries in the Western Pacific Region; on the other hand, a number of countries in the Region have economic or other ties with Africa. Risk assessments conducted by the World Health Organization (WHO) Regional Office for the Western Pacific ${ }^{4}$ suggest that the possibility of imported EVD cases to the Region is not high, but if it occurs the consequences would be major. The consequences of an introduction, particularly in developing country settings such as those found in many Pacific island countries, would likely be severe. Many of these countries are characterized by poor health-care infrastructure, low health-seeking behaviour, crowded housing, close-knit yet extensive social networks, low levels of sanitation and hygiene, low health literacy and challenging infrastructure. In light of this, a framework for action to respond to Ebola virus

disease for the Western Pacific Region was developed by the WHO Regional Office for the Western Pacific to assist unaffected countries and areas in the Region set priorities for preparedness to detect and respond to an outbreak of EVD. The framework for action provides a useful checklist for countries in the Region to determine their preparedness in key areas related to EVD. ${ }^{5}$

As part of its support to Member States, and within the context of the International Health Regulations (IHR 2005) ${ }^{6}$ and the Asia Pacific Strategy for Emerging Diseases (APSED 2010, a strategy for capacity-building for public health events in the South-East Asia and Western Pacific regions), ${ }^{7}$ the WHO Regional Office for the Western Pacific recently conducted two activities to assess EVD preparedness in the Region. The first of these was an online survey administered to the National IHR Focal Point in each Member State. The second was a simulation exercise conducted with the participation of the National IHR Focal Points in Member States, WHO country offices and the WHO Regional Office. The findings of these two activities can help National IHR Focal Points and the WHO Regional Office identify strengths and challenges regarding the preparedness level in the Region and help partners identify areas of potential support.

\footnotetext{
Emerging Disease Surveillance and Response Unit, Division of Health Security and Emergencies, World Health Organization Regional Office

for the Western Pacific, Manila, Philippines.

Office of the WHO Representative in Papua New Guinea, Port Moresby, Papua New Guinea.

* Both authors contributed equally in the writing of this paper.

Submitted: 12 December 2014; Published: 26 January 2015

doi: 10.5365/wpsar.2014.5.4.004
} 


\section{METHODS}

\section{Ebola preparedness survey}

Invitations to the web-based Ebola preparedness survey were sent to all 27 National IHR Focal Points in the WHO Western Pacific Region on 29 September 2014. The survey was not administered to non-self-governing areas within the Region, such as several Pacific island jurisdictions, as these do not have National IHR Focal Points. The survey consisted of self-reporting against 34 closed-ended ("yes" or "no") questions. Questions were related to the six components of the framework for action: (1) command and coordination; (2) surveillance, risk assessment and response; (3) laboratory; (4) clinical management, and infection prevention and control; (5) public health interventions, including points of entry measures; and (6) risk communication. Analysis was conducted for all countries and separately for Asian countries and Pacific island countries.

\section{Ebola simulation exercise}

An Ebola simulation exercise, which required National IHR Focal Points to respond quickly to incoming information by email regarding a potential EVD event, was conducted from 8 to 9 October 2014. Invitations to participate were sent to all 27 National IHR Focal Points in the Western Pacific Region.

Briefly, the exercise simulated the entry and detection of a travel-related EVD case in a local district in the National IHR Focal Points' country which required them to take rapid response actions. The exercise scenario and injects were designed to promote the following expected actions: (1) share national EVDrelevant guidelines and response plans; (2) provide technical advice on contact tracing, case management and patient transferral; (3) arrange specimen referral to WHO-recognized laboratories; (4) notify WHO of suspected and confirmed EVD cases under IHR (2005); and (5) draft the press release of the first announcement of an EVD case.

\section{RESULTS}

\section{Ebola preparedness survey}

Results of the survey are shown in Table 1 . Twenty-six National IHR Focal Points (96\%) completed the survey: Australia, Brunei Darussalam, Cambodia, China, Cook Islands, Fiji, Japan, Kiribati, the Lao People's Democratic Republic, Malaysia, the Marshall Islands, the Federated States of Micronesia, Mongolia, New Zealand, Niue, Palau, Papua New Guinea, the Philippines, the Republic of Korea, Samoa, Singapore, Solomon Islands, Tonga, Tuvalu, Vanuatu and Viet Nam.

The components of EVD preparedness with the highest number of positive responses for all countries on average were risk communication (86\%) and command and coordination (84\%). The lowest-scoring component was laboratory (60\%). Asian countries and Pacific island countries differed in their overall responses, with the Pacific island countries registering lower than their Asian counterparts in every component.

Within the command and coordination component, most Asian and Pacific island countries had plans and structures in place in case of an EVD event; $92 \%$ of Asian countries had tested or planned to test those plans, but only $8 \%$ of Pacific island countries had done so (prior to the simulation exercise described below).

In the surveillance, risk assessment and response component, an EVD surveillance protocol had been developed and disseminated to public health officials and hospitals in $92 \%$ of Asian countries compared to $15 \%$ of Pacific island countries. An investigation protocol had been developed in $100 \%$ of Asian countries compared to $31 \%$ of Pacific island countries. National rapid response teams (RRTs) had been briefed or trained on EVD response in $92 \%$ of Asian countries and countryspecific risk assessments conducted in $85 \% ; 31 \%$ of Pacific island countries reported completing either of the same preparedness actions.

In regard to laboratory preparedness, in-country capacity to diagnose suspected EVD cases was limited to Asian countries alone (10/13). However, 77\% of Asian countries and $54 \%$ of Pacific island countries reported having a referral arrangement in place, and $77 \%$ of countries in each subregion reported having staff trained to package specimens for referral.

For clinical management and infection, prevention and control, most countries in both subregions had a designated isolation facility, had an infection prevention and control guidelines in place and had given staff appropriate training. However, compared to $77 \%$ of 


\section{Table 1. Responses to a web-based survey of Ebola preparedness by Asian, Pacific island and all countries and areas, * WHO Western Pacific Region, 2014}

\begin{tabular}{|c|c|c|c|c|c|c|}
\hline Questions & $\begin{array}{l}\text { All countries } \\
\text { number/total }\end{array}$ & $\%$ & $\begin{array}{l}\text { Asian countries } \\
\text { number/total }\end{array}$ & $\%$ & $\begin{array}{l}\text { Pacific countries } \\
\text { number/total }\end{array}$ & $\%$ \\
\hline \multicolumn{7}{|l|}{ Command and coordination } \\
\hline $\begin{array}{l}\text { Is there a national public health emergency response } \\
\text { plan (or equivalent) that can be used to contain } \\
\text { potential EVD events? }\end{array}$ & $25 / 26$ & 96 & $13 / 13$ & 100 & $12 / 13$ & 92 \\
\hline $\begin{array}{l}\text { Is there an incident command and coordination } \\
\text { structure set up to coordinate the containment of } \\
\text { potential EVD events? }\end{array}$ & $25 / 26$ & 96 & $13 / 13$ & 100 & $12 / 13$ & 92 \\
\hline $\begin{array}{l}\text { If an EVD case were to occur, would EOC in } \mathrm{MOH} \text { be } \\
\text { activated for response operations? }^{\dagger}\end{array}$ & $23 / 25$ & 92 & $11 / 12$ & 92 & $12 / 13$ & 92 \\
\hline $\begin{array}{l}\text { Has an EVD exercise/simulation been planned or } \\
\text { conducted? }\end{array}$ & $13 / 26$ & 50 & $12 / 13$ & 92 & $1 / 13$ & 8 \\
\hline Average & & 84 & & 96 & & 71 \\
\hline \multicolumn{7}{|l|}{ Surveillance, risk assessment and response } \\
\hline $\begin{array}{l}\text { Is } \mathrm{MOH} \text { monitoring the EVD situation in } \\
\text { West Africa? }\end{array}$ & $26 / 26$ & 100 & $13 / 13$ & 100 & $13 / 13$ & 100 \\
\hline $\begin{array}{l}\text { Has awareness of EVD been raised among health- } \\
\text { care workers? }\end{array}$ & $24 / 26$ & 92 & $12 / 13$ & 92 & $12 / 13$ & 92 \\
\hline $\begin{array}{l}\text { Has a country-specific risk assessment been } \\
\text { conducted? }\end{array}$ & $15 / 26$ & 58 & $11 / 13$ & 85 & $4 / 13$ & 31 \\
\hline $\begin{array}{l}\text { Are the existing event-based surveillance/indicator- } \\
\text { based surveillance systems used to detect potential } \\
\text { EVD cases? }\end{array}$ & $22 / 26$ & 85 & $13 / 13$ & 100 & $9 / 13$ & 69 \\
\hline $\begin{array}{l}\text { Has a case/event definition of EVD been developed in } \\
\text { your country? }\end{array}$ & $20 / 26$ & 77 & $13 / 13$ & 100 & $7 / 13$ & 54 \\
\hline $\begin{array}{l}\text { Has an EVD surveillance protocol been developed and } \\
\text { disseminated to public health officials and hospitals? }\end{array}$ & $14 / 26$ & 54 & $12 / 13$ & 92 & $2 / 13$ & 15 \\
\hline $\begin{array}{l}\text { Is there a reporting mechanism in hospitals to report } \\
\text { unusual events/cases to public health authorities? }\end{array}$ & $25 / 26$ & 96 & $13 / 13$ & 100 & $12 / 13$ & 92 \\
\hline $\begin{array}{l}\text { Has an investigation protocol/flow chart for EVD cases } \\
\text { been developed? }\end{array}$ & $17 / 26$ & 65 & $13 / 13$ & 100 & $4 / 13$ & 31 \\
\hline Are contact tracing/management guidelines available? & $22 / 26$ & 85 & $12 / 13$ & 92 & $10 / 13$ & 77 \\
\hline $\begin{array}{l}\text { Has the national RRT been briefed/trained on EVD } \\
\text { response? }^{\dagger}\end{array}$ & $15 / 25$ & 60 & $11 / 12$ & 92 & $4 / 13$ & 31 \\
\hline $\begin{array}{l}\text { Is there a mechanism to notify WHO of a suspected } \\
\text { case through the International Health Regulations } \\
\text { (IHR 2005)? }\end{array}$ & $26 / 26$ & 100 & $13 / 13$ & 100 & $13 / 13$ & 100 \\
\hline Average & & 79 & & 96 & & 63 \\
\hline \multicolumn{7}{|l|}{ Laboratory } \\
\hline $\begin{array}{l}\text { Is there in-country laboratory capacity for testing } \\
\text { suspected EVD specimens? }\end{array}$ & $10 / 26$ & 38 & $10 / 13$ & 77 & $0 / 13$ & 0 \\
\hline $\begin{array}{l}\text { Has a referral arrangement with a WHO-recognized } \\
\text { laboratory for Ebola virus diagnosis been made? }\end{array}$ & $17 / 26$ & 65 & $10 / 13$ & 77 & $7 / 13$ & 54 \\
\hline $\begin{array}{l}\text { Are there staff trained to organize air shipment of } \\
\text { specimens (Ebola virus or any other agent) according } \\
\text { to IATA guidelines? }\end{array}$ & $20 / 26$ & 77 & $10 / 13$ & 77 & $10 / 13$ & 77 \\
\hline Average & & 60 & & 77 & & 44 \\
\hline
\end{tabular}

* Asian countries included Australia, Brunei Darussalam, Cambodia, China, Japan, the Lao People's Democratic Republic, Malaysia, Mongolia, New Zealand, Papua New Guinea, the Philippines, the Republic of Korea, Singapore and Viet Nam; Pacific island countries included Cook Islands, Fiji, Kiribati, the Marshall Islands, the Federated States of Micronesia, Niue, Palau, Samoa, Solomon Islands, Tonga, Tuvalu and Vanuatu.

$\dagger$ Based on available survey results.

EOC, Emergency Operations Centre; EVD, Ebola virus disease; IATA, International Air Transport Association; MOH, Ministry of Health; POE, points of entry; RRT, rapid response team. 
Table 1. Responses to a web-based survey of Ebola preparedness by Asian, Pacific island and all countries and areas,* WHO Western Pacific Region, 2014 (continued)

\begin{tabular}{|c|c|c|c|c|c|c|}
\hline Questions & $\begin{array}{l}\text { All countries } \\
\text { number/total }\end{array}$ & $\%$ & $\begin{array}{l}\text { Asian countries } \\
\text { number/total }\end{array}$ & $\%$ & $\begin{array}{l}\text { Pacific countries } \\
\text { number/total }\end{array}$ & $\%$ \\
\hline \multicolumn{7}{|l|}{ Clinical management \& infection prevention and control } \\
\hline $\begin{array}{l}\text { Has your country designated a hospital/hospitals for } \\
\text { EVD cases? }\end{array}$ & $22 / 26$ & 85 & $13 / 13$ & 100 & $9 / 13$ & 69 \\
\hline $\begin{array}{l}\text { Have infection prevention and control guidelines that } \\
\text { can be used for EVD response been developed and } \\
\text { disseminated? }\end{array}$ & $19 / 26$ & 73 & $10 / 13$ & 77 & $9 / 13$ & 69 \\
\hline $\begin{array}{l}\text { Is there an adequate supply of personal protective } \\
\text { equipment in your country for EVD rapid response and } \\
\text { containment operations? }\end{array}$ & $12 / 26$ & 46 & $10 / 13$ & 77 & $2 / 13$ & 15 \\
\hline $\begin{array}{l}\text { Has training on personal protective equipment for } \\
\text { health-care workers been given in the designated } \\
\text { hospital/hospitals? }\end{array}$ & $20 / 26$ & 77 & $13 / 13$ & 100 & $7 / 13$ & 54 \\
\hline Average & & 70 & & 89 & & 52 \\
\hline \multicolumn{7}{|l|}{ Public health interventions including POE measures } \\
\hline $\begin{array}{l}\text { Have public health measures been identified to } \\
\text { contain potential EVD events? }{ }^{+}\end{array}$ & $19 / 25$ & 76 & $12 / 12$ & 100 & $7 / 13$ & 54 \\
\hline $\begin{array}{l}\text { Is there a public health emergency contingency plan } \\
\text { in place at POE that can be used for EVD event } \\
\text { management? }\end{array}$ & $20 / 26$ & 77 & $11 / 13$ & 85 & $9 / 13$ & 69 \\
\hline $\begin{array}{l}\text { Have POE staff been sensitized/briefed on appropriate } \\
\text { action to manage suspected EVD cases in arriving } \\
\text { travellers? }\end{array}$ & $17 / 26$ & 65 & $13 / 13$ & 100 & $4 / 13$ & 31 \\
\hline $\begin{array}{l}\text { Is there a separate area for rapid assessment and } \\
\text { isolation of suspected EVD cases at POE? }\end{array}$ & $20 / 26$ & 77 & $12 / 13$ & 92 & $8 / 13$ & 62 \\
\hline $\begin{array}{l}\text { Is there a mechanism at POE for referral of ill } \\
\text { travellers to designated hospitals? }\end{array}$ & $23 / 26$ & 88 & $13 / 13$ & 100 & $10 / 13$ & 77 \\
\hline $\begin{array}{l}\text { Is there a protocol to monitor and manage returning } \\
\text { travellers with Ebola-like symptoms? }\end{array}$ & $16 / 26$ & 62 & $13 / 13$ & 100 & $3 / 13$ & 23 \\
\hline $\begin{array}{l}\text { Is EVD-related information for both incoming and } \\
\text { outgoing travellers available at POE? }\end{array}$ & $15 / 26$ & 58 & $11 / 13$ & 85 & $4 / 13$ & 31 \\
\hline Average & & 72 & & 95 & & 50 \\
\hline \multicolumn{7}{|l|}{ Risk communication } \\
\hline $\begin{array}{l}\text { Has the relevant EVD information been disseminated } \\
\text { to the general public? }\end{array}$ & $20 / 26$ & 77 & $11 / 13$ & 85 & $9 / 13$ & 69 \\
\hline $\begin{array}{l}\text { Is there a risk communication plan in place to } \\
\text { communicate with stakeholders and the public during } \\
\text { an EVD event? }\end{array}$ & $21 / 26$ & 81 & $13 / 13$ & 100 & $8 / 13$ & 62 \\
\hline $\begin{array}{l}\text { Is there a procedure/mechanism in place to rapidly } \\
\text { disseminate EVD information and health messages? }\end{array}$ & $24 / 26$ & 92 & $13 / 13$ & 100 & $11 / 13$ & 85 \\
\hline $\begin{array}{l}\text { Is there a designated spokesperson in } \mathrm{MOH} \text { for } \\
\text { emerging diseases/outbreaks? }\end{array}$ & $24 / 26$ & 92 & $13 / 13$ & 100 & $11 / 13$ & 85 \\
\hline $\begin{array}{l}\text { Is there a mechanism in place for first announcement } \\
\text { (if an EVD case were to occur)? }\end{array}$ & $23 / 26$ & 88 & $12 / 13$ & 92 & $11 / 13$ & 85 \\
\hline Average & & 86 & & 95 & & 77 \\
\hline
\end{tabular}

* Asian countries included Australia, Brunei Darussalam, Cambodia, China, Japan, the Lao People's Democratic Republic, Malaysia, Mongolia, New Zealand, Papua New Guinea, the Philippines, the Republic of Korea, Singapore and Viet Nam; Pacific island countries included Cook Islands, Fiji, Kiribati, the Marshall Islands, the Federated States of Micronesia, Niue, Palau, Samoa, Solomon Islands, Tonga, Tuvalu and Vanuatu.

$\dagger$ Based on available survey results.

EOC, Emergency Operations Centre; EVD, Ebola virus disease; IATA, International Air Transport Association; MOH, Ministry of Health; POE, points of entry; RRT, rapid response team. 
Asian countries only $15 \%$ of Pacific island countries had adequate personal protective equipment to actually manage a potential EVD case.

There was great discrepancy between Asian countries and Pacific island countries with regard to public health intervention measures at points of entry. All Asian countries had briefed points of entry staff on the management of suspected EVD in arriving passengers, all had a protocol in place to monitor and manage returning travellers with EVD-like symptoms and $85 \%$ had EVD-related information available for both incoming and outgoing travellers at points of entry. In the same respective categories, $31 \%, 23 \%$ and $31 \%$ of Pacific island countries reported having completed those preparations.

Risk communication capacity was high in both groups, with few components requiring further strengthening; Pacific island countries demonstrated a median level of EVD public awareness dissemination $(69 \%)$ and development of risk communication plans for the event of an EVD outbreak (62\%).

\section{Ebola simulation exercise}

Twenty-three National IHR Focal Points participated in the exercise; 11 from Asian countries on 8 October and 12 from Pacific island countries on 9 October. Countries participating in the exercise were: Brunei Darussalam, Cambodia, China, Cook Islands, Fiji, Japan, Kiribati, the Lao People's Democratic Republic, Malaysia, the Marshall Islands, the Federated States of Micronesia, Mongolia, Niue, New Zealand, Palau, the Philippines, the Republic of Korea, Samoa, Singapore, Solomon Islands, Tuvalu, Vanuatu and Viet Nam. Five of the National IHR Focal Points (Fiji, Kiribati, the Federated States of Micronesia, the Republic of Korea and Vanuatu) participated but could not complete the exercise due to communication, technical or personnel constraints. The National IHR Focal Points of Australia, Nauru, Papua New Guinea and Tonga did not participate.

The exercise demonstrated that Asian countries are well advanced in the preparation of national EVD response plans or relevant guidelines. During the exercise, all 11 Asian countries were able to share the existing national EVD guidelines/response plans that had been developed in-country. Some Asian countries had developed very comprehensive response plans which included key components of emergency response coordination and communication. In contrast, only $30 \%$ of Pacific island countries could share EVD-relevant guidelines developed prior to the exercise.

All Asian country National IHR Focal Points were able to provide technical guidance to the simulated local public health unit on patient referral, contact tracing and case management. Some countries provided very technical and detailed advice and necessary assistance that could be beneficial at the local level. All Pacific island countries were able to provide advice on patient referral, contact tracing and management as well, but there were some technical inaccuracies with the materials provided by some Pacific island countries.

During the exercise, all Asian country and Pacific island country National IHR Focal Points were able to facilitate laboratory testing of simulated EVD specimens, although there was some minor confusion about referral mechanisms. National IHR Focal Points from two Asian countries with EVD diagnostic capacity declared that specimens only required in-country testing, while others understood the need for referral but were not clear about where specimens should be shipped. WHO requests that, at least initially, clinical specimens diagnosed in-country be shipped to specific WHO-recognized laboratories for confirmation.

Under IHR (2005), suspected or confirmed cases of EVD should be notified to WHO. Fifteen countries notified WHO of the individual meeting a suspected case definition in the scenario (9/11 Asian countries; 6/10 Pacific island countries), and 17 notified WHO of the confirmed case (9/11 Asian countries; 8/10 Pacific island countries). In total, $91 \%$ (10/11) of the Asian countries and $80 \%(8 / 10)$ of Pacific island countries notified WHO of either suspected or confirmed EVD cases.

Sixteen of the participating National IHR Focal Points (from 10/11 Asian countries and 6/10 Pacific island countries) were able to share a draft first announcement upon simulated confirmation of the EVD case, but difficulties were noted in some countries' ability to develop a well-written press release, especially in Pacific island countries. 


\section{DISCUSSION}

The survey and simulation exercise suggest that there is a good overall preparedness level in the Western Pacific Region in the event of an imported case of EVD. However, a number of areas still require further strengthening before the Region can efficiently and effectively respond to potential EVD events, including laboratory testing arrangements; clinical management and infection prevention and control; and public health intervention measures, particularly at points of entry. Importantly, the survey and exercise demonstrated that several countries were not completely ready to facilitate specimen testing. It was not expected that all countries (especially Pacific island countries) would have the appropriate facilities and biosafety infrastructure for in-country testing of suspected EVD specimens, but an adequate referral mechanism to facilitate specimen testing should be in place. Countries need to ensure fully that agreements have been made with WHO-recognized laboratories for confirmatory testing, have sufficiently trained staff to safely and correctly package suspected specimens and that export arrangements for dangerous goods have been prepared so that shipments will be smooth and efficient.

Both the survey and the simulation exercise highlight the unique situation and requirements of Pacific island countries compared to Asian countries and emphasize that special considerations for Pacific island countries are needed in terms of EVD preparedness, in particular specimen testing, interventions at points of entry and availability of personal protective equipment. The implications of the survey and exercise should extend beyond stakeholders in health, as the experience in West Africa has shown that an extensive EVD outbreak impacts and necessitates a response from the whole of society.

As the survey was based on country self-reporting, it is difficult to objectively assess whether the results provided are a true reflection of country capacity. To some extent, this was addressed by having the simulation exercise, which sought to elicit objective evidence of certain preparedness capacities such as the ability to quickly produce guidance documents. Still, many capacities were unable to be tested due to the tabletop nature of the simulation. This further underscores the need for in-country functional or full-scale exercises to truly assess capacity.
A limitation of the simulation exercise was that the personnel available to participate varied from one country to another. For example, one country had 20 participants, while another had only one to two participants as it was a national holiday. Thus, the performance in the exercise may not have been a true reflection of that country's actual capacity.

The survey and simulation exercise did not include the non-sovereign areas of the Pacific (e.g. American Samoa, Guam and French Polynesia); therefore, the preparedness levels in these areas could not be assessed. While many of the issues faced by these areas are likely similar to those encountered in their developing country neighbours, it is expected that they would have access to resources from their metropolitan governments (in this case, the United States and France, respectively). Other countries in the Region with close political ties to developed countries (e.g. Papua New Guinea to Australia, and the northern Micronesian countries to the United States) would probably benefit from similar support if an outbreak were to occur in their jurisdictions. It is in the interest of regional security for all that high-resource countries increase their support to developing countries; should an outbreak of EVD occur in an inadequately prepared developing country, patients seeking better care would likely flee to their high-resource, traditional allies. It would be far more cost-effective and politically acceptable for all countries involved to prevent such outbreaks in the first place.

The EVD outbreak in West Africa has served as a high alert to all countries against becoming complacent about the threat of emerging diseases and as an opportunity to test the public health and social systems that countries have put in place to deal with such threats. Many of the assessed elements of preparedness referenced here were generic capacities that could be used for a variety of events, not solely an EVD outbreak. Generic capacities allow for the quick adaptation of surveillance and response systems to novel or (re-)emerging pathogens, such as Ebola virus; this is particularly critical in developing country settings, such as the Pacific island countries, in which it is simply not feasible to have vertical approaches to each novel disease. APSED provides a foundation for generic public health emergency system strengthening based on its focus areas and contributions towards achieving IHR (2005) core capacities, but more remains to be 
done. Donors and technical partners can use the results of the preparedness survey and simulation exercise to prioritize urgent support to Member States to further advance these capacities, particularly in the areas of laboratory, case management, infection prevention and control and points of entry, particularly in the Pacific subregion. Further EVD-specific preparedness can be achieved through application of the framework for action. $^{5}$

\section{Conflicts of interest}

None declared.

\section{Funding}

None.

\section{Acknowledgements}

The WHO Regional Office for the Western Pacific Ebola Emergency Support Team acknowledges all Member States in the Western Pacific for their contribution and support to the Ebola preparedness survey and Ebola simulation exercise.

Other members of the WHO Western Pacific Region Ebola Emergency Support Team when the survey and exercise were conducted: Kotaro Tanaka,
Selenic Dubravka, May Chiew, Christine Cool, Samantha Chapman, Christophe Delaude and Janet Mina.

\section{References:}

1. Ebola Virus Disease Fact Sheet No 103, September 2014. Geneva, World Health Organization, 2014 (http://who.int/mediacentre/ factsheets/fs103/en/, accessed 10 November 2014).

2. Ebola Response Roadmap Situation Report. 8 October 2014. Geneva, World Health Organization, 2014 (http://apps.who.int/ iris/bitstream/10665/136020/1/roadmapsitrep_80ct2014_eng. pdf?ua =1, accessed 13 November 2014).

3. Cases of Ebola diagnosed in the United States. Atlanta, Centers for Disease Control and Prevention (CDC), 2014 (http://www.cdc. gov/vhf/ebola/outbreaks/2014-west-africa/united-states-importedcase.html, accessed 13 November 2014).

4. Ebola virus disease: Risk assessment in the Western Pacific Region - 09 October 2014. Manila, World Health Organization Regional Office for the Western Pacific, 2014 (http://www.wpro. who.int/outbreaks_emergencies/wpr_ra_ebola_09oct2014. pdf?ua $=1$, accessed 13 November 2014).

5. Preparedness for a potential outbreak of Ebola virus disease: a framework for action in the Western Pacific Region. Manila, World Health Organization Regional Office for the Western Pacific, 2014 (http://www.wpro.who.int/outbreaks_emergencies/wpro ebola/en/, accessed 13 November 2014).

6. International Health Regulations. (2005), Second edition. Geneva, World Health Organization, 2008 (http://www.who.int/ ihr/9789241596664/en/, accessed 13 November 2014).

7. Asia Pacific Strategy for Emerging Diseases (APSED, 2010). Manila, World Health Organization Regional Office for the Western Pacific, 2011 (http://www.wpro.who.int/emerging_diseases/ APSED2010/en/, accessed 13 November 2014). 


\section{World Health \\ Western Pacific Region}

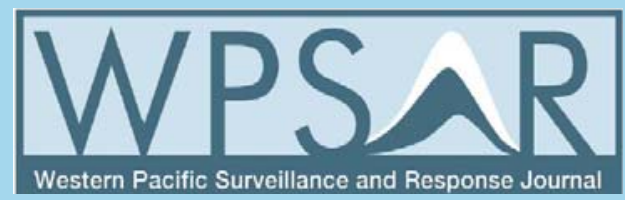

wpsar@wpro.who.int I www.wpro.who.int/wpsar 